\title{
Mapping Integration Definition for Function Modeling (IDEFO) Model into CASE Data Interchange Format (CDIF) Transfer File
}

Igor Simakhodskiy Guest Researcher

U.S. DEPARTMENT OF COMMERCE

Technology Administration National Institute of Standards and Technology

Gaithersburg, MD 20899

QC

100

.056

N0. 5719 



\section{NISTIR 5719}

\section{Mapping Integration Definition for Function Modeling (IDEFO) Model into CASE Data Interchange Format (CDIF) Transfer File}

\section{Igor Simakhodskiy Guest Researcher}

U.S. DEPARTMENT OF COMMERCE Technology Administration National Institute of Standards and Technology

Gaithersburg, MD 20899

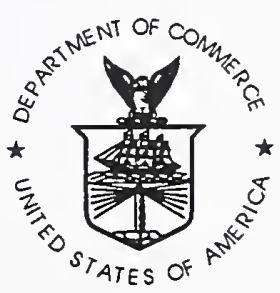

U.S. DEPARTMENT OF COMMERCE Ronald H. Brown, Secretary

TECHNOLOGY ADMINISTRATION

Mary L. Good, Under Secretary for Technology

NATIONAL INSTITUTE OF STANDARDS

AND TECHNOLOGY

Arati Prabhakar, Director 
$$
\text { . }
$$ 


\section{Table of Contents}

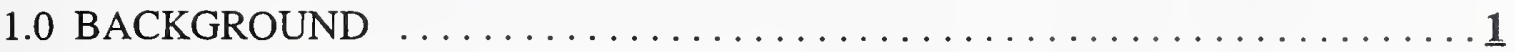

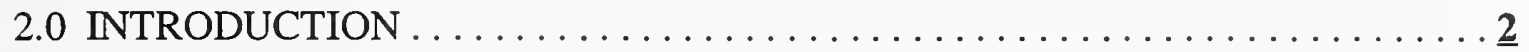

3.0 CDIF - CASE DATA INTERCHANGE FORMAT $\ldots \ldots \ldots \ldots \ldots \ldots \ldots \ldots$

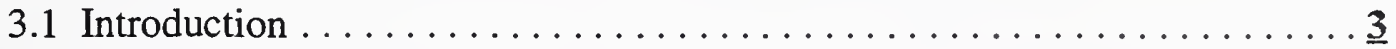

3.2 Using CDIF to Transfer IDEF Models $\ldots \ldots \ldots \ldots \ldots \ldots \ldots \ldots \ldots \ldots \ldots \ldots$

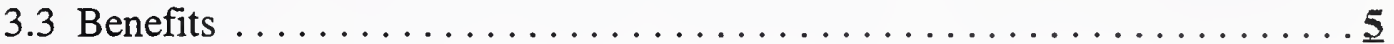

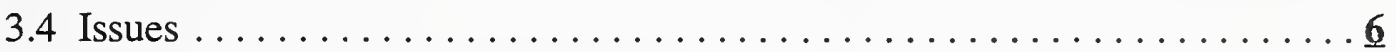

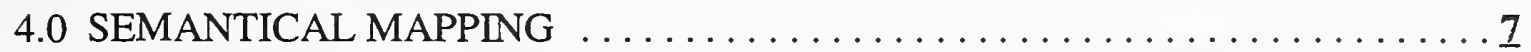

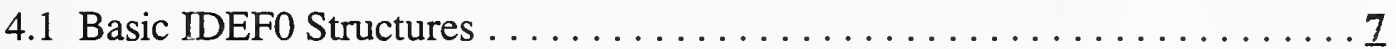

4.1.1 IDEF0 Top-Level Context Diagram $\ldots \ldots \ldots \ldots \ldots \ldots \ldots \ldots$

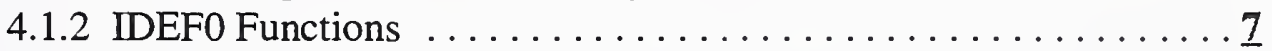

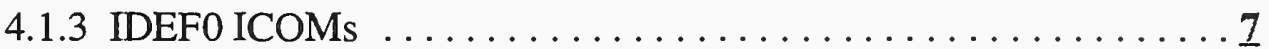

4.1.4 Putting Together ICOMs and Functions $\ldots \ldots \ldots \ldots \ldots \ldots$

4.1.5 Process Decomposition: Crossing an Abstraction Level ........ 2

4.1.6 Example: Mapping A-0 Diagram into CDIF Transfer File ..... 10

4.1.7 Node References . . . . . . . . . . . . . . . . . 22

4.1 .8 Model Notes . . . . . . . . . . . . . . . . . . . . . 22

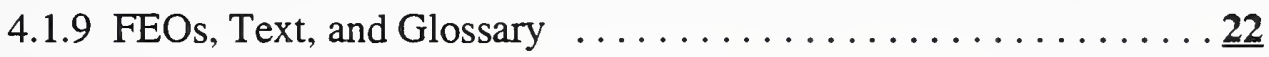

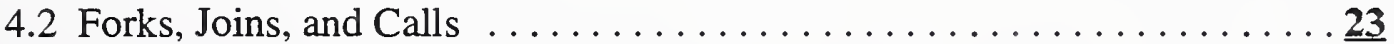

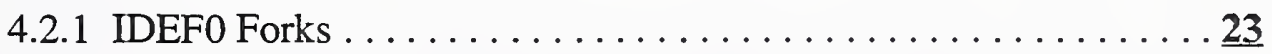

4.2.1.1 "A Copy" Fork ...................

4.2.1.2 "A Portion" Fork .................... $\underline{\underline{26}}$

4.2.1.3 "The Sum" Fork .................... . 29

4.2.2 IDEF0 Joins . . . . . . . . . . . . . . . . . . . 34

4.2.2.1 "A Copy" Join ........................

4.2.2.2 "A Portion" Join ......................

4.2.2.3 "The Sum" Join . . . . . . . . . . . . . . . . . . . 40

4.2.3 IDEF0 Calls . .................

4.2.3.1 "Another Data Model" Call . . ............... $4 \mathbf{4 5}$

4.2.3.2 "A Document" Call . . . . . . . . . . . . . . . . 47

4.2.3.3 SBT (Split By Type) Call . . . . . . . . . . . 48

4.2.3.4 A Drop in the Level of Abstraction Call ......... $\underline{\mathbf{5 2}}$

4.3 Process/Flow Decomposition ........................

4.3.1 "Blind Copy" Flow Decomposition .............. 5 .

4.3.2 "A Copy" Flow Decomposition ................

4.3.3 "Blind Portion" Flow Decomposition ............... 79 
4.3.4 "A Portion" Flow Decomposition $\ldots \ldots \ldots \ldots \ldots \ldots \ldots \ldots$

4.3.5 "Blind Sum" Flow Decomposition $\ldots \ldots \ldots \ldots \ldots \ldots \ldots \underline{\underline{81}}$

4.3.6 "The Sum" Flow Decomposition . . . . . . . . . . . . . 82

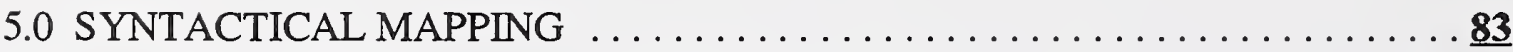

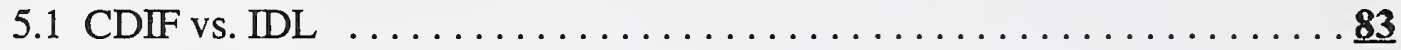

5.1 .1 Coordinate Frame $\ldots \ldots \ldots \ldots \ldots \ldots \ldots \ldots \ldots \ldots \ldots \ldots$

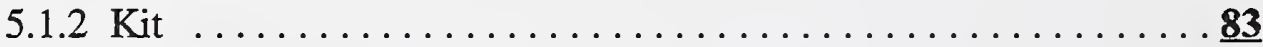

5.1 .3 Diagram ..................

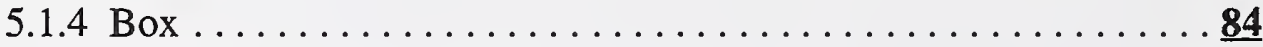

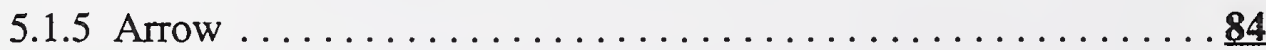

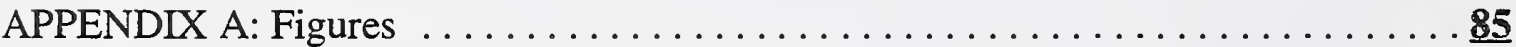

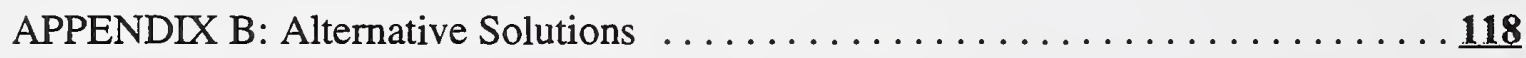

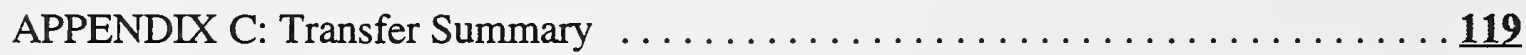

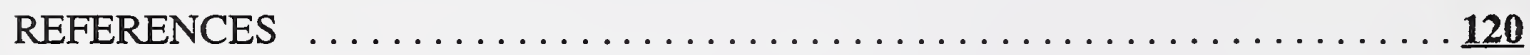




\section{IDEF0 MAPPING}

\section{Structure of this Document:}

This document is organized into the following sections:

\section{Section 1: Background}

This gives some background information on IDEF0, CDIF, and the idea of a general information exchange between different tools.

\section{Section 2: Introduction}

This introduces the audience to this document and gives some more information about CDIF.

\section{Section 3: CDIF - Case Data Interchange Format}

This explains the CDIF structure and shows how CDIF can be used as a standard means for exchanging information between IDEF tools.

\section{Section 4: Semantical Mapping}

This gives the full description of the semantical mapping of IDEF0 graphical models (diagrams) into CDIF transfer files.

\section{Section 5: Syntactical Mapping}

This gives the full description of the syntactical mapping of IDEF0 graphical models into CDIF transfer files.

\section{Appendix A: Figures}

This shows all the figures used in the report as well as the figures with the graphical representation of the mapping of some IDEF0 structures.

\section{Appendix B: Alternative Solutions}

This gives other ways to map specific IDEF0 components into the CDIF transfer file.

\section{Appendix C: Transfer Summary}

This gives a summary of the IDEF0 to CDIF transfer and gives a list of additional CDIF structures needed for the $100 \%$ transfer.

\section{References}

This provides the references to the documents used in creating this report. 


\section{Conventions:}

Italics: CDIF meta-entities, meta-relationships, meta-attributes.

Italics \& Bold: values for meta-attributes. 


\subsection{BACKGROUND}

The Integration Definition for Function Modeling (IDEF0) is a standard modeling technique used in the logical definition of an organization's data resources. The standard defines the syntax and semantics of the language constructs used in creating a function model. With the acceptance of the standard, more vendors are providing support for this technique.

One serious limitation to the standard is the lack of a formal standard mechanism for transferring information between different IDEF tools and between IDEF tools and other tools supporting different modeling techniques. This leaves the users of IDEF tools at a great disadvantage. A user is restricted to using a particular tool provided by a particular vendor thus seriously hindering the reusability of the information represented in a model. This may also force an organization to mandate the use of a specific tool. This infringes on a users freedom to use a tool that best supports both the work and the users particular style.

One solution is the use of a standard method to exchange data between tools. The CASE Data Interchange Format (CDIF) Family of Standards is primarily designed to be used as a description of a mechanism for transferring information between CASE tools.

Vendors who support the CDIF standards need only define the exchange mechanism once for their particular tool. This would allow the exchange of information developed using a particular vendor's tool with other vendors' tools that support the CDIF standards. Not only would the use of these standards allow users of IDEF tools to exchange data between IDEF tools, it could also support the exchange of information captured by an IDEF tool with other CASE tools.

The ability to freely exchange data between tools benefits an organization by promoting the reuse of information resources. It also allows the IDEF users to focus more on the analysis of business and information problems instead of problems and limitations of tools. 


\subsection{INTRODUCTION}

This document will describe a conceptual mapping of an IDEF0 model into the CDIF transfer file. The document will cover both syntax and semantics of the model. This work will demonstrate that it is possible to use the standard exchange for moving information between different tools.

This ATP funded work was to research a standard transfer mechanism for IDEF0 models. The document covers the Normative section of IDEF0.

CDIF has defined a Meta-model which captures the syntax and semantics of data used by numerous tools. The Meta-model has a set of different subject areas allowing the vendors to express the contents of their tool repositories using a standard format. This provides a common definition of model data for communicating between different tools.

CDIF can also be used in situations where vendor defined data that does not map to the Meta-model (extensible data) needs to be transferred. The CDIF Family of Standards includes a Framework for Modeling and Extensibility Standard which allows a vendor to define new meta-objects used to capture this extensible data. It also defines the relationships which link the newly defined meta-objects to the existing standard Metamodel. 


\subsection{CDIF - CASE DATA INTERCHANGE FORMAT}

\subsection{Introduction}

CDIF consists of a set of standards referred to as the CDIF Family of Standards which provides a standard means for exchanging information between CASE tools. These standards can move both the syntax and semantics of information captured within a particular tool.

The current set of standards is broken into four sections: Overview, Framework, Integrated Meta-model, and Transfer Format. This report uses information defined in the Integrated Meta-model section.

The Integrated Meta-model is divided into partitions or subject areas. Each subject area defines a meta-model for specific types of information that could be developed using a CASE tool. For example, the Data Flow Modeling Subject Area defines meta-data for process flow models, the Data Model Subject Area defines meta-data captured in data models, and Presentation Location and Connectivity Subject Area is used to capture the syntactic data about a particular model.

The components of CDIF that were used for this mapping were Common Subject Area, Data Flow Model Subject Area, and Presentation Location and Connectivity Subject Area. All three subject areas were necessary for capturing the types of information created by the IDEF0 tools.

The Common Subject Area applies to all of the CDIF Meta-model standards. It is used to capture information relevant to all system development objects. It addresses such items as who created the object, when it was last updated and possible synonyms for the object.

The Data Flow Modeling Subject Area provides support for modeling the input-processoutput view of a system. It provides all the concepts needed for data flows into and out of processes, with associated data stores and external entities.

The Presentation Location and Connectivity Subject Area covers information that provides either spatial position information for specific instances, or relative position in the directed graph sense (nature of the connectivity between related objects), relating to the graphical representation of semantic objects. 


\subsection{Using CDIF to Transfer IDEF Models}

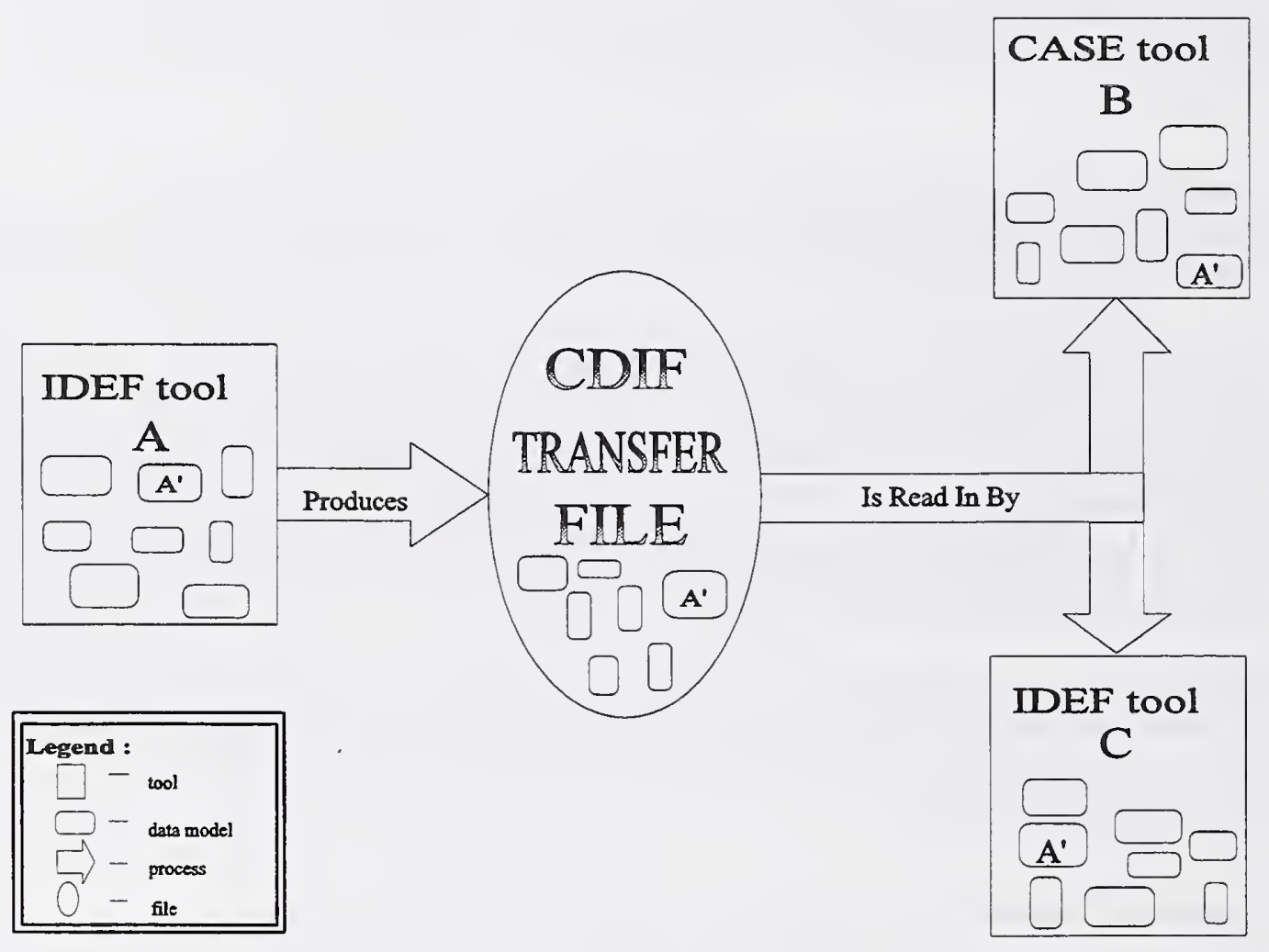

Figure 1. Transferring IDEF Models Using CDIF

It is very important to understand the basic concepts of a CDIF based transfer. There exists an IDEF tool A with some process models. This tool produces a CDIF transfer file for a particular process model $\mathrm{A}^{\prime}$. This file can then be read in by another IDEF tool $\mathrm{C}$ or a CASE tool B. The CDIF transfer file preserves the concepts of $\mathrm{A}^{\prime}$ through the transfer into the other tools. $\mathrm{A}^{\prime}$ can now be accessed, printed, and modified by a user of tool $\mathrm{C}$ or a user of tool $B$ without any influence on the model $A^{\prime}$ in tool $A$. 


\subsection{Benefits}

Many benefits can be gained from the use of the standard transfer mechanism. One great benefit to the users of IDEF tools is the ability to reuse already created models. Much time and money is currently spent on developing models, when a lot of information represented on these models already exist.

To promote reuse organizations are developing procedures for managing this information. CDIF can be used to assist in this effort by providing the standard exchange mechanism.

Another benefit to the user is the ability to use tools best suited for a particular job. Models created by one user for a project using a particular tool can be read and modified by other users using different tools. This can also save time and money by reducing the effort spent on purchasing and learning new tools.

If an organization chooses to exchange information between different tools it usually invests in customized converters to get the job done. These converters may not work on updated versions of those specific tools. Also additional converters would be needed for expanding this information exchange between other tools. As the number of tools increases so does the problem of managing the transfers. The benefit to using CDIF as a solution to this problem is that CDIF reduces or even eliminates the need for customized converters for the transfer of information between different tools.

A benefit to IDEF tool vendors is that their tools would be able to interface with multiple tools thus increasing marketability. As the figure 1 indicates, an IDEF tool stores the contents of a model in a file which then can be converted into a CDIF transfer file using the converter developed by the IDEF tool vendor. Through a converter developed by another vendor the CDIF transfer file can then be changed into a file format which is used by the specific CASE or IDEF tool. Since the transfer mechanism is standard, vendors could spend more time on enhancing and developing features for their tools.

Moreover, there is another great benefit to using CDIF as a transfer format. CDIF transfers the syntax and semantics of a model. This is very important to IDEF users because more than the diagrams are transferred between tools. It would be incorrect to simply map a box from an IDEF (or CASE) tool to a box in some other CASE tool without checking the semantics for a box in both tools because those boxes may have different meanings. 


\subsection{Issues}

One issue of transfer formats is that there is no guarantee for $100 \%$ transfer of data. This occurs because one tool might not support all the concepts of another tool, in which case some information may be lost. Another situation would be if a tool handles multiple meanings of a concept of another tool. For example, one tool might have a classification of calls, while another tool just handles one concept of a call (refer to the section 4.2 .3 for types of calls).

Another issue is that vendors currently are not implementing the CDIF Family of Standards. The reason is that most of the CDIF work has yet to be approved as official standards. Also tool vendors may have a difficult time realizing the benefit of using CDIF. Users of tools should stress the importance of having a standard transfer format. 


\subsection{SEMANTICAL MAPPING}

\subsection{Basic IDEF0 Structures}

\subsubsection{IDEF0 Top-Level Context Diagram}

To map an IDEF0 top-level context diagram into a CDIF transfer file, create an instance of the CDIF meta-entity DataFlowModel and DFMProcessDefinition. There is no metaentity DataFlowModelDefinition in CDIF; therefore we must use DFMProcessDefinition in order to capture the true meaning of the model. This DFMProcessDefinition will then contain the top-level function A0 and the ICOMs going in and out of A0. Create an instance of the CDIF meta-entity DFMProcessDefinition for A- 0 , and connect this instance with the instance of the CDIF meta-relationship DataFlowModel via the CDIF meta-relationship DataFlowModel.HasRoot.DFMProcessDefinition.

\subsubsection{IDEF0 Functions}

To map an IDEF0 function (process) into a CDIF transfer file, create instances of the CDIF meta-entities: DFMProcessDefinition and DFMProcess. Then create instances of the CDIF meta-relationships: DFMProcess.References.DFMProcessDefinition and DFMProcessDefinition.Contains.DFMProcess. The CDIF meta-relationship DFMProcess.References.DFMProcessDefinition allows the function (process) to be connected with its definition. The CDIF meta-relationship DFMProcessDefinition.Contains.DFMProcess allows a process definition to declare its structure.

\subsubsection{IDEF0 ICOMs}

To map an IDEFO ICOM into a CDIF transfer file, create instances of CDIF meta-entities: FlowDefinition and Flow. Then create an instance of the CDIF meta-relationship Flow.References.FlowDefinition. This meta-relationship allows a flow to be linked with its definition. An instance of the CDIF meta-relationship DFMProcessDefinition.Contains.Flow is also needed to be created. This metarelationship, like DFMProcessDefinition.Contains.DFMProcess, is needed to build the architecture of that DFMProcessDefinition which contains the DFMProcess or Flow. 


\subsubsection{Putting Together ICOMs and Functions}

In CDIF, flows (ICOMs) cannot enter (or leave) a process directly: they need to go through a FlowPort -- a subtype of a Port.

The meta-entity Port is a generic, abstract concept for DefinitionObjects (supertype of DFMProcessDefinition and FlowDefinition) to declare their interfaces to other objects. There are two types of interfaces to be specified: the formal interface to the outside of the DefinitionObject is formed by all instances of subtypes of Port which have the value of its meta-attribute IsFormal set to True. To specify the actual Ports that reference the formal Ports, instances of subtypes of Port are used which have the value of the metaattribute IsFormal set to False.

Because the interface of DFMProcesses is accessed by Flows only, concrete meta-entity FlowPort was provided instead of the abstract meta-entity Port. Flows cannot "cross" the boundary of a Process without using the concept of a FlowPort.

A Port has the following subtypes:
o FlowInputPort
o ConstraintPort
o FlowOutputPort
o SupportPort

These subtypes correspond directly to the IDEFO ICOMs:

$\begin{array}{ll}\text { Input } & \text { - FlowInputPort } \\ \text { Control } & \text { - ConstraintPort } \\ \text { Output } & \text { - FlowOutputPort } \\ \text { Mechanism } & \text { - SupportPort }\end{array}$

Like in IDEF0, a CDIF flow is just a stream. A CDIF flow is not an Input flow or an Output flow without a port. The IDEF0 role of a flow is represented in the CDIF concept of a port. 


\subsubsection{Process Decomposition: Crossing an Abstraction Level}

In CDIF, DefinitionObjects are not contained by other DefinitionObjects. Only ComponentObjects are contained by DefinitionObjects. Every DFMProcessDefinition used in the mapping represents a line between levels of abstraction. A

DFMProcessDefinition itself is not contained by a particular abstraction level. However, a DFMProcessDefinition contains all the components (CDIF meta-entities) which exist within an abstraction level "below the abstraction line" (see Figure 2). The dashed lines on the Figure 2 are the lines between different abstraction levels. A DFMProcessDefinition contains various CDIF Meta-entities (CDIF ME) such as Flow, FlowInputPort, FlowOutputPort, ConstraintPort, SupportPort, EquivalenceSet, ReferencedElement, DFMProcess, and others.)

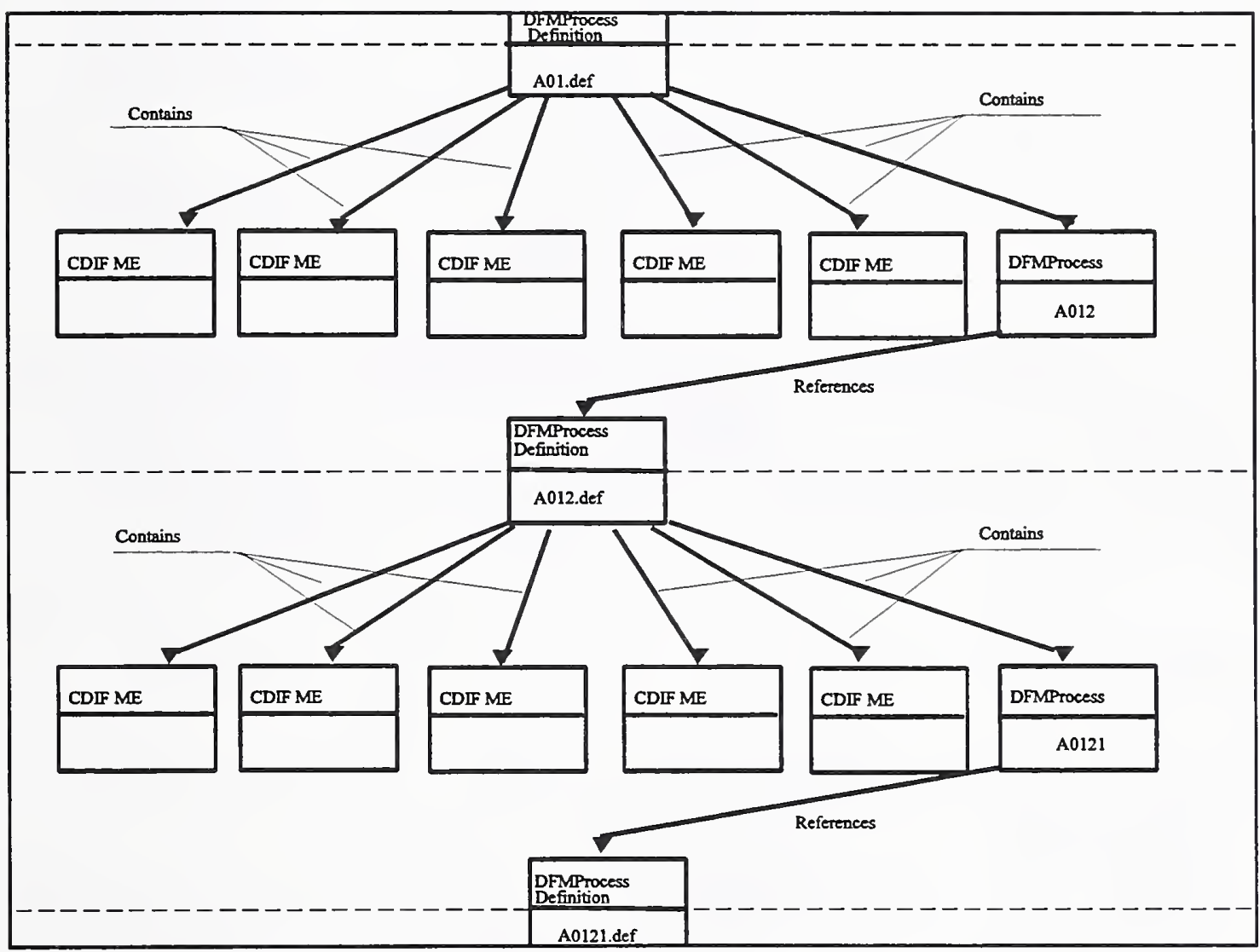

Figure 2. Crossing an Abstraction Level 


\subsubsection{Example: Mapping A-0 Diagram into CDIF Transfer File}

The figure below is a typical A-0 diagram. We are at the highest level of abstaction at which we see the purpose and viewpoint of the model. At this level we need to define the whole model (or diagram) A-0 in CDIF. This is done using CDIF meta-entity DataFlowModel. The purpose and viewpoint of the model can be captured using the local meta-attribute FullDescription of the DataFlowModel.

Below we will find the mapping of this diagram into the CDIF transfer file.

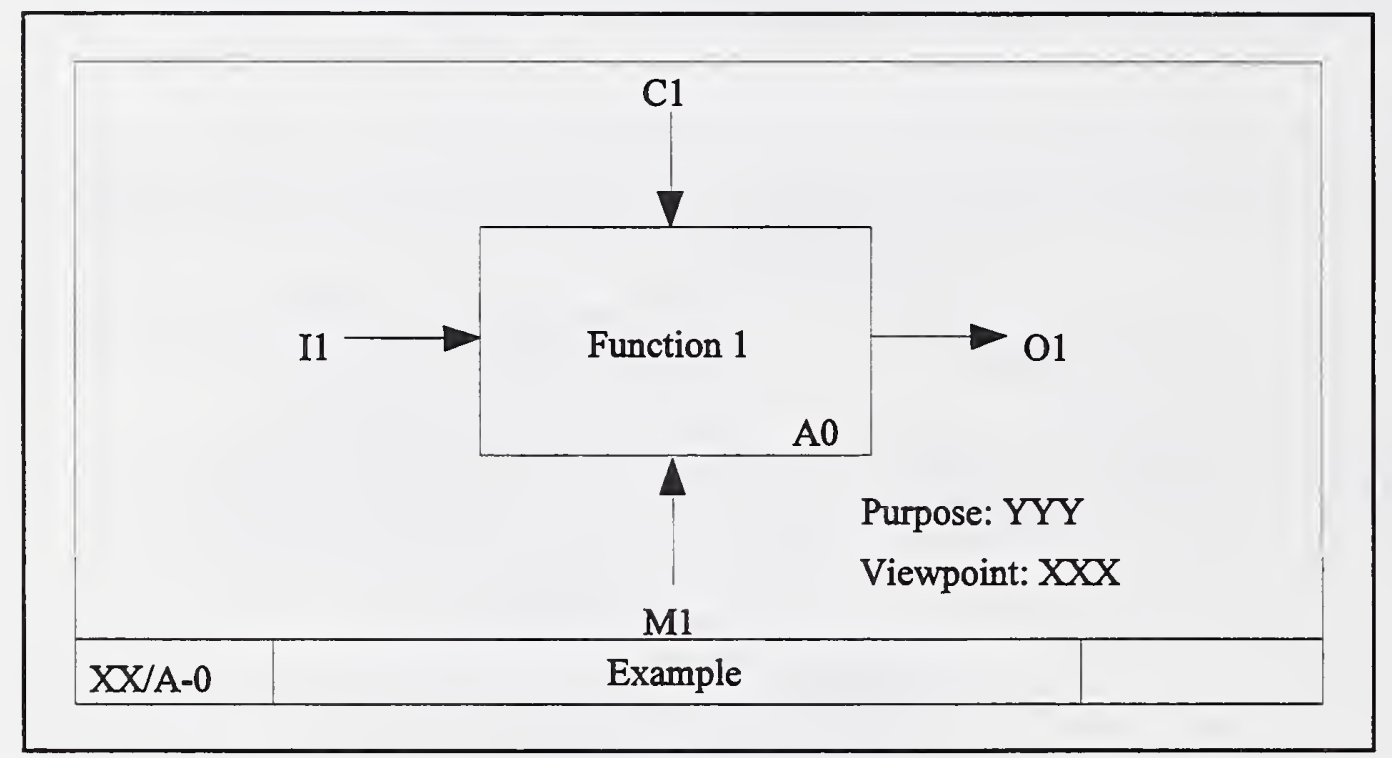

Figure 3. A-0 Diagram

\section{CDIF Transfer File}

Meta-entity ......... DataFlowModel

Meta-attributes

CDIFIdentifier .........dfm_example

Name ............. "Example"

FullDescription ........ "Purpose: YYY. Viewpoint: XXX."

Meta-entity ........ DFMProcessDefinition

Meta-attributes

CDIFIdentifier .........pd_A-0

Name ............. "A-0_def" 
Meta-relationship ..... DataFlowModel.HasRoot.DFMProcessDefinition Meta-attributes

CDIFIdentifier .........h hr

SourceEntity ..........dfm_example

DestinationEntity ........pd_A-0

Meta-entity DFMProcess

Meta-attributes

CDIFIdentifier .........dfmp_A0

Name ............ "A0"

BriefDescription ........."Function 1"

Meta-relationship ..... DFMProcessDefinition.Contains.DFMProcess

Meta-attributes

CDIFIdentifier ........ pd_A-0.c.dfmp_A0

SourceEntity .......... pd_A-0

DestinationEntity .......dfmp_A0

Meta-entity ......... DFMProcessDefinition

Meta-attributes

CDIFIdentifier ...........pd_A0

Name ..............A0_def"

Meta-relationship ..... . DFMProcess.References.DFMProcessDefinition

Meta-attributes

CDIFIdentifier $\ldots \ldots \ldots$. dfmp_A0.r.pd_A0

SourceEntity ..........dfmp_A0

DestinationEntity $\ldots \ldots \ldots$ pd_A0

Meta-entity ......... Flow

Meta-attributes

CDIFIdentifier .........f f_Il

Name .............. "I1"

ContextDescription ...... This is a generic input for the function 1 .

Meta-relationship ..... DFMProcessDefinition.Contains.Flow

Meta-attributes

CDIFIdentifier $\ldots \ldots \ldots$. pd_A-0.c.f_I1

SourceEntity .......... pd_A-0

DestinationEntity .........f_I1 
Meta-entity ......... FlowInputPort

Meta-attributes

CDIFIdentifier ......... fip_I1a

IsFormal ............ FALSE

Meta-relationship ..... . DFMProcessDefinition.Contains.FlowInputPort

Meta-attributes

CDIFIdentifier ...........pd_A-0.c.fip_I1a

SourceEntity .......... pd_A-0

DestinationEntity ........ fip_Ila

Meta-relationship ..... FlowInputPort.Consumes.Flow

Meta-attributes

CDIFIdentifier ........ fip_I1a.c.f_I1

SourceEntity ......... fip_Ila

DestinationEntity ........f_I1

Meta-entity ......... EquivalenceSet

Meta-attributes

CDIFIdentifier ........es_I1

Meta-relationship ..... . DFMProcessDefinition.Contains.EquivalenceSet

Meta-attributes

CDIFIdentifier .........pd_A-0.c.es_I1

SourceEntity ............pd_A-0

DestinationEntity .......es_I1

Meta-relationship ..... EquivalenceSet.HasMember.FlowInputPort

Meta-attributes

CDIFIdentifier ........e. es_I1.hm.fip_Ila

SourceEntity .......... es_I1

DestinationEntity ........ fip_I1a

Meta-entity ......... ReferencedElement

Meta-attributes

CDIFIdentifier ......... re_I1

Meta-relationship ..... . DFMProcessDefinition.Contains.ReferencedElement

Meta-attributes

CDIFIdentifier ........pd_A-0.c.re_I1

SourceEntity ..........pd_A-0

DestinationEntity ....... re_I1 
Meta-relationship ..... EquivalenceSet.HasMember.ReferencedElement

Meta-attributes

CDIFIdentifier .......e. es_I1.hm.re_I1

SourceEntity ........... es_I1

DestinationEntity ........ re_I1

Meta-relationship ..... ReferencedElement.DefinesPath.ComponentObject

Meta-attributes

CDIFIdentifier .........re_I1.dp.dfmp_A0

SourceEntity ........... re_I1

DestinationEntity ........dfmp_A0

SequenceNumber ....... 1

Meta-entity ......... FlowDefinition

Meta-attributes

CDIFIdentifier ........fd_I1

Name .............."I1_def"

Meta-relationship ..... Flow.References.FlowDefinition

Meta-attributes

CDIFIdentifier ........f_Il.r.fd_I1

SourceEntity ........... f_I1

DestinationEntity ....... fd_Il

Meta-relationship ..... FlowInputPort.References.FlowDefinition

Meta-attributes

CDIFIdentifier $\ldots \ldots \ldots$ fip_I1a.r.fd_I1

SourceEntity .......... fip_Ila

DestinationEntity ....... fd_Il

Meta-entity ......... FlowInputPort

Meta-attributes

CDIFIdentifier ........ fip_Ilf

IsFormal ........... TRUE

Meta-relationship ..... . DFMProcessDefinition.Contains.FlowInputPort

Meta-attributes

CDIFIdentifier ..........pd_A0.c.fip_Ilf

SourceEntity .......... pd_A0

DestinationEntity ....... fip_I1f 
Meta-relationship ..... FlowInputPort.References.FlowDefinition

Meta-attributes

CDIFIdentifier ....... fip_I1f.r.fd_I1

SourceEntity .......... fip_Ilf

DestinationEntity .......fd_I1

Meta-relationship ..... ReferencedElement.DefinesPath.ComponentObject

Meta-attributes

CDIFIdentifier ......... re_I1.dp.fip_Ilf

SourceEntity .......... re_I1

DestinationEntity ........ fip_I1f

SequenceNumber .......2

Meta-entity .......... Flow

Meta-attributes

CDIFIdentifier ........f. $\mathrm{f} 1$

Name ............."C1"

ContextDescription ...... This is a generic control for the function 1.

Meta-relationship ..... DFMProcessDefinition.Contains.Flow

Meta-attributes

CDIFIdentifier ........ pd_A-0.c.f_C1

SourceEntity .......... pd_A-0

DestinationEntity ........f_C1

Meta-entity ......... ConstraintPort

Meta-attributes

CDIFIdentifier ..........cp_Cla

IsFormal ............ FALSE

Meta-relationship ..... . DFMProcessDefinition.Contains.ConstraintPort

Meta-attributes

CDIFIdentifier $\ldots \ldots \ldots$ pd_A-0.c.cp_Cla

SourceEntity ..........pd_A-0

DestinationEntity ........cp_C1a

Meta-relationship ..... ConstraintPort.Consumes.Flow

Meta-attributes

CDIFIdentifier ........cp_C1a.c.f_C1

SourceEntity ...........cp_Cla

DestinationEntity .......f_c1 
Meta-entity ......... EquivalenceSet

Meta-attributes

CDIFIdentifier ........e.es_C1

Meta-relationship ..... . DFMProcessDefinition.Contains.EquivalenceSet

Meta-attributes

CDIFIdentifier $\ldots \ldots \ldots$. pd_A-0.c.es_C1

SourceEntity ............ pd_A-0

DestinationEntity .......es_C1

Meta-relationship ..... EquivalenceSet.HasMember.ConstraintPort

Meta-attributes

CDIFIdentifier ........es_Cl.hm.cp_Cla

SourceEntity ........... es_C1

DestinationEntity .........cp_Cla

Meta-entity ......... ReferencedElement

Meta-attributes

CDIFIdentifier .........re_C1

Meta-relationship ..... . DFMProcessDefinition.Contains.ReferencedElement

Meta-attributes

CDIFIdentifier $\ldots \ldots \ldots$ pd_A-0.c.re_C1

SourceEntity ..........pd_A-0

DestinationEntity ........ re_C1

Meta-relationship ..... EquivalenceSet.HasMember.ReferencedElement

Meta-attributes

CDIFIdentifier ........es_C1.hm.re_C1

SourceEntity .......... es_C1

DestinationEntity ....... re_C1

Meta-relationship ..... ReferencedElement.DefinesPath.ComponentObject

Meta-attributes

CDIFIdentifier ........ re_C1.dp.dfmp_A0

SourceEntity .......... re_Cl

DestinationEntity .......dfmp_A0

SequenceNumber ....... 1

Meta-entity ......... FlowDefinition

Meta-attributes

CDIFIdentifier ........fd_Cl

Name ............"Cl_def" 
Meta-relationship ..... Flow.References.FlowDefinition

Meta-attributes

CDIFIdentifier .........f_C1.r.fd_C1

SourceEntity ............ $\mathrm{f} 1$

DestinationEntity .......fd_c1

Meta-relationship ..... ConstraintPort.References.FlowDefinition

Meta-attributes

CDIFIdentifier .........cp_Cla.r.fd_C1

SourceEntity ...........cp_Cla

DestinationEntity ........fd_C1

Meta-entity ........ ConstraintPort

Meta-attributes

CDIFIdentifier ........cp_C1f

IsFormal $\ldots \ldots \ldots \ldots$ TRUE

Meta-relationship ..... . DFMProcessDefinition.Contains.ConstraintPort

Meta-attributes

CDIFIdentifier .........pd_A0.c.cp_C1f

SourceEntity ........... pd_A0

DestinationEntity ........cp_C1f

Meta-relationship ..... ConstraintPort.References.FlowDefinition

Meta-attributes

CDIFIdentifier .........cp_C1f.r.fd_C1

SourceEntity ...........cp_C1f

DestinationEntity .......fd_Cl

Meta-relationship ..... ReferencedElement.DefinesPath.ComponentObject

Meta-attributes

CDIFIdentifier .........re_C1.dp.cp_C1f

SourceEntity .......... re_C1

DestinationEntity .........cp_C1f

SequenceNumber .......2

Meta-entity ......... Flow

Meta-attributes

CDIFIdentifier ........f.O.

Name ............. "O1"

ContextDescription ...... This is a generic output for the function 1 . 
Meta-relationship ..... . DFMProcessDefinition.Contains.Flow

Meta-attributes

CDIFIdentifier $\ldots \ldots \ldots$ pd_A-0.c.f_O1

SourceEntity .......... pd_A-0

DestinationEntity ........ f_O1

Meta-entity ......... FlowOutputPort

Meta-attributes

CDIFIdentifier ......... fop_Ola

IsFormal FALSE

Meta-relationship ..... . DFMProcessDefinition.Contains.FlowOutputPort

Meta-attributes

CDIFIdentifier ..........pd_A-0.c.fop_Ola

SourceEntity .......... pd_A-0

DestinationEntity ....... fop_Ola

Meta-relationship ..... . FlowOutputPort.Produces.Flow

Meta-attributes

CDIFIdentifier ......... fop_Ola.p.f_O1

SourceEntity .......... fop_Ola

DestinationEntity $\ldots . . \ldots$ f_O1

Meta-entity ......... EquivalenceSet

Meta-attributes

CDIFIdentifier ........es_ol

Meta-relationship ..... . DFMProcessDefinition.Contains.EquivalenceSet

Meta-attributes

CDIFIdentifier $\ldots \ldots \ldots$ pd_A-0.c.es_O1

SourceEntity .......... pd_A-0

DestinationEntity .......e. es_O1

Meta-relationship ..... EquivalenceSet.HasMember.FlowOutputPort

Meta-attributes

CDIFIdentifier .........es_O1.hm.fop_O1a

SourceEntity ........... es_O1

DestinationEntity ........ fop_Ola

Meta-entity ......... ReferencedElement

Meta-attributes

CDIFIdentifier .........re_O1 
Meta-relationship ...... DFMProcessDefinition.Contains.ReferencedElement Meta-attributes

CDIFIdentifier $\ldots \ldots \ldots$ pd_A-0.c.re_O1

SourceEntity .......... pd_A-0

DestinationEntity $\ldots . .$. re_O1

Meta-relationship ..... EquivalenceSet.HasMember.ReferencedElement

Meta-attributes

CDIFIdentifier ........es_Oel.hm.re_O1

SourceEntity .......... es_O1

DestinationEntity .......re_re

Meta-relationship ..... ReferencedElement.DefinesPath.ComponentObject

Meta-attributes

CDIFIdentifier .........re_Ol.dp.dfmp_A0

SourceEntity .........re.Ore

DestinationEntity ......dfmp_A0

SequenceNumber ........ 1

Meta-entity ......... FlowDefinition

Meta-attributes

CDIFIdentifier .......fdd_O1

Name ............. "O1_def"

Meta-relationship ...... Flow.References.FlowDefinition

Meta-attributes

CDIFIdentifier $\ldots \ldots \ldots$. f_O1.r.fd_O1

SourceEntity ........... f_O1

DestinationEntity .......fd_O1

Meta-relationship ..... . FlowOutputPort.References.FlowDefinition

Meta-attributes

CDIFIdentifier ........ fop_Ola.r.fd_O1

SourceEntity ......... fop_Ola

DestinationEntity ....... fd_O1

Meta-entity . ......... FlowOutputPort

Meta-attributes

CDIFIdentifier ........ fop_Olf

IsFormal ........... TRUE 
Meta-relationship ..... DFMProcessDefinition.Contains.FlowOutputPort

Meta-attributes

CDIFIdentifier ..........pd_A0.c.fop_O1f

SourceEntity .......... pd_A0

DestinationEntity ........ fop_O1f

Meta-relationship ...... FlowOutputPort.References.FlowDefinition

Meta-attributes

CDIFIdentifier ......... fop_O1f.r.fd_O1

SourceEntity .......... fop_Olf

DestinationEntity $\ldots \ldots \ldots$ fd_O1

Meta-relationship ..... ReferencedElement.DefinesPath.ComponentObject

Meta-attributes

CDIFIdentifier $\ldots \ldots \ldots$. re_O1.dp.fop_O1f

SourceEntity .......... re_O1

DestinationEntity ........ fop_O1f

SequenceNumber ........2

Meta-entity .......... Flow

Meta-attributes

CDIFIdentifier ........f_M1

Name ............."M1"

ContextDescription ...... This is a generic mechanism for the function 1 .

Meta-relationship ..... . DFMProcessDefinition.Contains.Flow

Meta-attributes

CDIFIdentifier $\ldots \ldots \ldots$ pd_A-0.c.f_M1

SourceEntity ............pd_A-0

DestinationEntity ........f_M1

Meta-entity ......... SupportPort

Meta-attributes

CDIFIdentifier ..........sp_Mla

IsFormal ............ FALSE

Meta-relationship ..... DFMProcessDefinition.Contains.SupportPort

Meta-attributes

CDIFIdentifier $\ldots \ldots \ldots$. pd_A-0.c.sp_M1a

SourceEntity ............pd_A-0

DestinationEntity ........sp_Mla 
Meta-relationship .... . SupportPort.Consumes.Flow

Meta-attributes

CDIFIdentifier .......... sp_M1a.c.f_M1

SourceEntity .............sp_M1a

DestinationEntity ........ f_M1

Meta-entity ......... EquivalenceSet

Meta-attributes

CDIFIdentifier .........es_M1

Meta-relationship ..... . DFMProcessDefinition.Contains.EquivalenceSet

Meta-attributes

CDIFIdentifier $\ldots \ldots \ldots$. pd_A-0.c.es_M1

SourceEntity .......... pd_A-0

DestinationEntity .......es_M1

Meta-relationship ..... EquivalenceSet.HasMember.SupportPort

Meta-attributes

CDIFIdentifier ........ es_M1.hm.sp_M1a

SourceEntity .......... es_M1

DestinationEntity .........sp_Mla

Meta-entity ......... ReferencedElement

Meta-attributes

CDIFIdentifier ......... re_M1

Meta-relationship ...... DFMProcessDefinition.Contains.ReferencedElement

Meta-attributes

CDIFIdentifier ........pd_A-0.c.re_M1

SourceEntity .......... pd_A-0

DestinationEntity ........re_M1

Meta-relationship ...... EquivalenceSet.HasMember.ReferencedElement

Meta-attributes

CDIFIdentifier ........es_M1.hm.re_M1

SourceEntity .......... es_M1

DestinationEntity .......re_M1

Meta-relationship ..... ReferencedElement.DefinesPath.ComponentObject

Meta-attributes

CDIFIdentifier ......... re_M1.dp.dfmp_A0

SourceEntity ..........re_M1

DestinationEntity .......dfmp_A0

SequenceNumber ........ 
Meta-entity ......... FlowDefinition

Meta-attributes

CDIFIdentifier ........fd_M1

Name "M1_def"

Meta-relationship ..... Flow.References.FlowDefinition

Meta-attributes

CDIFIdentifier ........f_M1.r.fd_M1

SourceEntity ........... f_M1

DestinationEntity .......fd_M1

Meta-relationship ..... SupportPort.References.FlowDefinition

Meta-attributes

CDIFIdentifier ..........sp_Mla.r.fd_M1

SourceEntity ............sp_Mla

DestinationEntity .......fd_.M1

Meta-entity ......... SupportPort

Meta-attributes

CDIFIdentifier ...........sp_M1f

IsFormal ........... TRUE

Meta-relationship ..... DFMProcessDefinition.Contains.SupportPort Meta-attributes

CDIFIdentifier .........pd_A0.c.sp_M1f

SourceEntity .......... pd_A0

DestinationEntity .........sp_Mlf

Meta-relationship ...... SupportPort.References.FlowDefinition

Meta-attributes

CDIFIdentifier .......... sp_M1f.r.fd_M1

SourceEntity ............sp_M1f

DestinationEntity .......fd_M1

Meta-relationship ..... ReferencedElement.DefinesPath.ComponentObject

Meta-attributes

CDIFIdentifier .........re_M1.dp.sp_M1f

SourceEntity .......... re_M1

DestinationEntity .........sp_Mlf

SequenceNumber .......2 


\subsubsection{Node References}

Each diagram in an IDEF0 model has a node reference, which is used to uniquely identify the diagram and its position in the model hierarchy. The node references is composed of the abbreviated model name and the diagram node number, separated by a slash (/). For example, a model named Example might be abbreviated as EX, and a node reference might then be EX/A132. References to a diagram in the same model may omit the model name abbreviation, using only the diagram node number.

Node references can be handled in two different ways in CDIF. The first and easiest way is to put the node reference in the local meta-attribute Name of the DFMProcess. But in this case we will need to capture the real name of the function in BriefDescription. The second way is to create a CDIF local meta-attribute NodeReference (TYPE: string) using the CDIF Framework for Modeling and Extensibility.

\subsubsection{Model Notes}

Even though model notes are optional, we would like to mention them in this report. Model notes are denoted by an integer inside a small square box. For a particular diagram, the note number is formed in a consecutive sequence, starting at 1. (Vertical pipes surrounding the note number are used as an alternative notation: $|n|$.)

The easiest way to represent a model note in CDIF is to put it into the ContextDescription of the DFMProcess for which this model note was created. The sequencing is presented using the local meta-attribute ContextIdentifier.

\subsubsection{FEOs, Text, and Glossary}

The node-numbering extension notation consists of a single letter appended to the associated node number to provide the basis for coordinating FEOs, text, and glossary terms. If there is more than one FEO, glossary term, or text page associated with given IDEF0 node, the pages should be designated with an additional number to uniquely identify each. For example, node numbers for the second glossary term shall contain "G2" for "second glossary" (e.g., A132G2).

If a FEO, text, or glossary term refers to the type of the process (not to the particular instance of this type, but to all the instances of this type), then this FEO, text, or glossary term can be captured using the local meta-attribute SpecificationText of the meta-entity DFMProcessDefinition. If, however, the FEO, text, or glossary term refers to the particular instance of the process, it can be captured using FullDescription of the DFMProcess. 


\subsection{Forks, Joins, and Calls}

\subsubsection{IDEFO Forks}

All the IDEF0 Forks can be categorized into three groups:

- "A Copy" Fork

- "A Portion" Fork

- "The Sum" Fork

\subsubsection{1 "A Copy" Fork}

\section{IDEFO Fork}

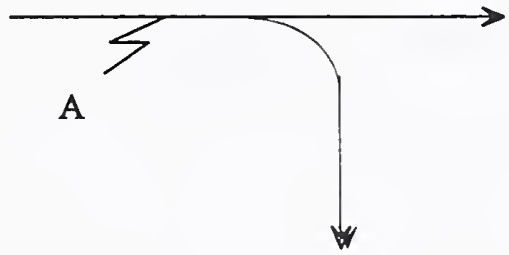

\section{Interpretation}

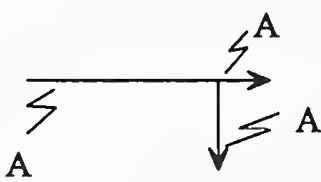

\section{CDIF Flow Split}

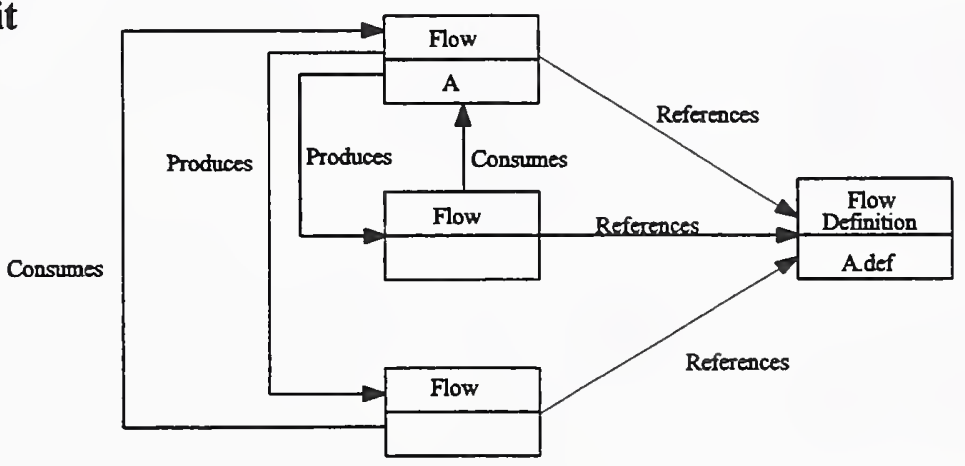

Figure 4. "A Copy" Fork 
The diagram above shows the "Copy" type of IDEF0 Fork and its mapping into the CDIF graphical representation of the transfer format. We need to create a FlowDefinition for A, and then reuse it for all the Flows. The following is the CDIF transfer file for the figure above. Since we have three instances of Flow A and we cannot have the same CDIFIdentifiers for all of them ${ }^{1}$, we will use single and double quoted letter A. In general, a single or double quoted letter which plays a role of a CDIFIdentifier will identify a particular instance of the flow indicated by that letter.

Meta-entity ............. FlowDefinition

Meta-attributes

CDIFIdentifier ..........fd_A

Name .............."A.def"

Meta-entity ......... Flow

Meta-attributes

CDIFIdentifier ......... f_A

Name ............. "A"

Meta-relationship ..... Flow.References.FlowDefinition

Meta-attributes

CDIFIdentifier ..........f_A.r.fd_A

SourceEntity ............A

DestinationEntity .......fd_A

Meta-entity ......... Flow

Meta-attributes

CDIFIdentifier ...............

Meta-relationship ..... Flow.References.FlowDefinition

Meta-attributes

CDIFIdentifier .........f_A'.r.fd_A

SourceEntity ...............

DestinationEntity ........fd_A

Meta-entity ......... Flow

Meta-attributes

CDIFIdentifier ........f.f"

${ }^{1}$ CDIFIdentifiers are unique. 
Meta-relationship ...... Flow.References.FlowDefinition

Meta-attributes

CDIFIdentifier ........f.fA".r.fd_A

SourceEntity ..........f_A"

DestinationEntity ....... fd_A

Meta-relationship ..... FlowProducerConsumer.Produces.Flow Meta-attributes

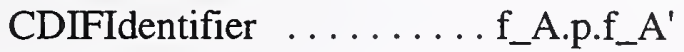

SourceEntity ...........f_A

DestinationEntity ........f_A

Meta-relationship ..... . FlowProducerConsumer.Produces.Flow

Meta-attributes

CDIFIdentifier .........f_A.p.f_A"

SourceEntity ............. f_A

DestinationEntity .......f.f."

Meta-relationship ..... . FlowProducerConsumer.Consumes.Flow

Meta-attributes

CDIFIdentifier ........f_A'.c.f_A

SourceEntity ...............

DestinationEntity ........ f_A

Meta-relationship ..... FlowProducerConsumer.Consumes.Flow Meta-attributes

CDIFIdentifier ........f.A".c.f_A

SourceEntity ..........f.A"

DestinationEntity ........f_A 


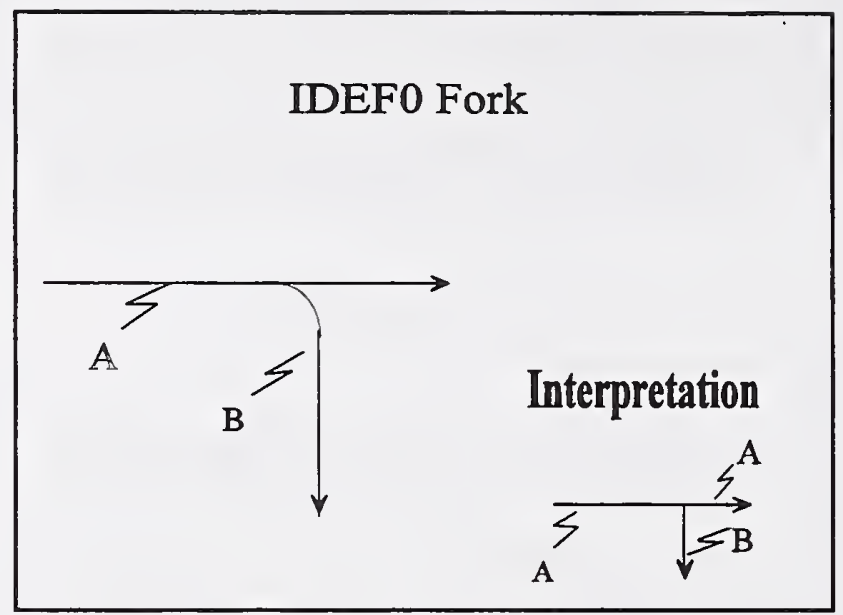

Figure 5. "A Portion" Fork

The above figure is another type of IDEF0 Fork. The flow B is created as a portion of the flow A. In this case, we need to create a FlowDefinition for A and a FlowDefinition for B. We will reuse the FlowDefinition for A to reference the second A flow. A graphical mapping of this type of fork can be found in Appendix A. The following is the CDIF transfer file for the Figure 5.

Meta-entity ......... FlowDefinition

Meta-attributes

CDIFIdentifier ........fd_A

Name .................def"

Meta-entity ......... Flow

Meta-attributes

CDIFIdentifier ........f.A

Name ............ "A"

Meta-relationship ..... Flow.References.FlowDefinition

Meta-attributes

CDIFIdentifier ........f.A.r.fd_A

SourceEntity ............ f_A

DestinationEntity .......fd_A

Meta-entity ......... FlowDefinition

Meta-attributes

CDIFIdentifier $\ldots \ldots \ldots$ fd_A:b 
Meta-entity ......... Flow

Meta-attributes

CDIFIdentifier .......... $\mathrm{f} b$

Meta-relationship ..... Flow.References.FlowDefinition

Meta-attributes

CDIFIdentifier .......f.b.r.fd_A:b

SourceEntity ......... f $\_b$

DestinationEntity $\ldots \ldots \ldots$ fd_A:b

Meta-relationship ..... FlowDefinition.Contains.Flow

Meta-attributes

CDIFIdentifier ........fd_A.c.f_b

SourceEntity ..........fd_A

DestinationEntity .......f f_b

Meta-entity ......... Flow

Meta-attributes

CDIFIdentifier .........f_A'

Meta-relationship ..... . Flow.References.FlowDefinition

Meta-attributes

CDIFIdentifier ........f_A'.r.fd_A

SourceEntity ...............

DestinationEntity ........fd_A

Meta-entity ......... Flow

Meta-attributes

CDIFIdentifier .......... $\mathrm{f}_{-} \mathrm{B}$

Meta-relationship ..... Flow.References.FlowDefinition

Meta-attributes

CDIFIdentifier ........f_B.r.fd_A:b

SourceEntity .......... $\mathrm{f} \_\mathrm{B}$

DestinationEntity .......fd_A:b

Meta-relationship ..... . FlowProducerConsumer.Produces.Flow

Meta-attributes

CDIFIdentifier ................f_A'

SourceEntity ............ f_A

DestinationEntity ......... $\mathrm{f}_{-} \mathrm{A}^{\prime}$ 
Meta-relationship ..... FlowProducerConsumer.Produces.Flow

Meta-attributes

CDIFIdentifier ........f_A.p.f_B

SourceEntity .............A

DestinationEntity ......... f_B

Meta-relationship ..... FlowProducerConsumer.Consumes.Flow

Meta-attributes

CDIFIdentifier ........ f_A'.c.f_A

SourceEntity ..............

DestinationEntity .......... f_A

Meta-relationship ..... FlowProducerConsumer.Consumes.Flow

Meta-attributes

CDIFIdentifier ........f_B.c.f_A

SourceEntity ........... $f_{-} B$

DestinationEntity ......... f_A

$1^{*}$ The following is needed to show semantically that the flow B is a part of the flow A, and that it's the part $b$ which is contained by the FlowDefinition $\mathrm{fd}_{-} \mathrm{A}^{2 * /}$

Meta-entity ......... EquivalenceSet

Meta-attributes

CDIFIdentifier .........es_b

Meta-relationship ...... EquivalenceSet.HasMember.Flow

Meta-attributes

CDIFIdentifier ........es_b.hm.f_B

SourceEntity .......... es_b

DestinationEntity ......... $\mathrm{f}_{-} \mathrm{B}$

Meta-entity ......... ReferencedElement

Meta-attributes

CDIFIdentifier $\ldots . . . .$. re_b

2 The capital letter $B$ indicates the actual flow B, whereas the small letter b is used to show the structure of the flow $A$ which consists of flow $b$ (and possibly some other flows). The capital B represents the actual flow $B$ from the diagram, whereas the small b represents the conceptual flow which is a part of the flow $A$. 
Meta-relationship ..... . EquivalenceSet.HasMember.ReferencedElement

Meta-attributes

CDIFIdentifier .........es_b.hm.re_b

SourceEntity ........... es_b

DestinationEntity ........re_b

Meta-relationship ..... ReferencedElement.DefinesPath.Flow

Meta-attributes

CDIFIdentifier .......... re_b.dp.f_A

SourceEntity ........... re_b

DestinationEntity .......... f_A

SequenceNumber ....... 1

Meta-relationship ...... ReferencedElement.DefinesPath.Flow

Meta-attributes

CDIFIdentifier .........re_b.dp.f_b

SourceEntity ...........re_b

DestinationEntity ........f_b

SequenceNumber .......2

\subsubsection{3 "The Sum" Fork}

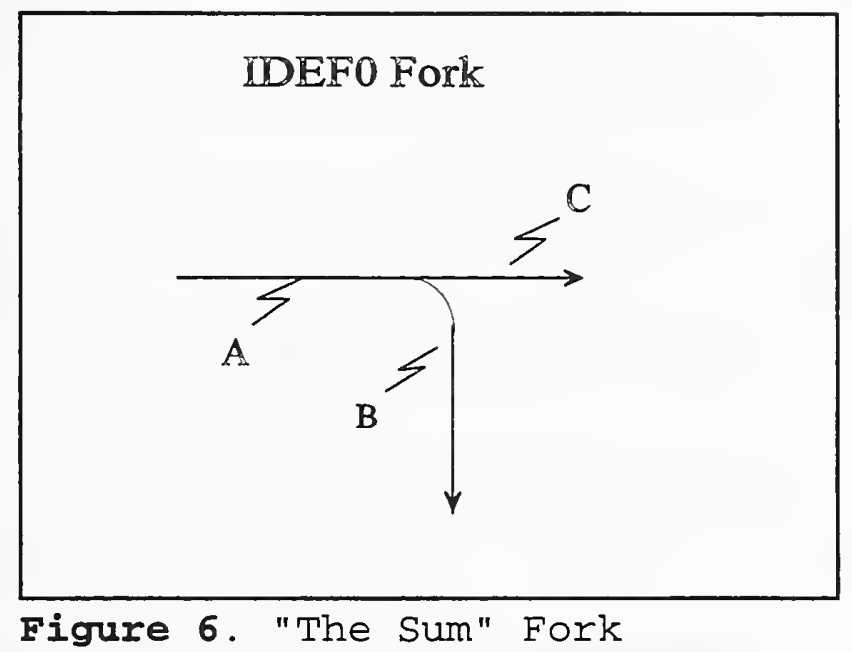

The above figure is the third type of IDEF0 Fork. The flow A contains flows B and C in its structure. We need to create a FlowDefinition for A, a FlowDefinition for B, and a FlowDefinition for C. A graphical representation of the mapping of this type of fork can be found in Appendix A. The following is the CDIF transfer file for the Figure 6. 
Meta-entity .......... FlowDefinition

Meta-attributes

CDIFIdentifier ........fd_A

Name .............."A.def"

Meta-entity ......... Flow

Meta-attributes

CDIFIdentifier ........f_A

Name ............." A" $^{\prime}$

Meta-relationship ...... Flow.References.FlowDefinition

Meta-attributes

CDIFIdentifier ........f_A.r.fd_A

SourceEntity .......... f_A

DestinationEntity $\ldots . \ldots$ fd_A

Meta-entity ......... FlowDefinition

Meta-attributes

CDIFIdentifier ........fd_A:b

Meta-entity .......... Flow

Meta-attributes

CDIFIdentifier .........f $\mathrm{f} b$

Meta-relationship ..... Flow.References.FlowDefinition

Meta-attributes

CDIFIdentifier ........f_b.r.fd_A:b

SourceEntity .......... f_b

DestinationEntity $\ldots \ldots \ldots$ fd_A:b

Meta-relationship ..... FlowDefinition.Contains.Flow

Meta-attributes

CDIFIdentifier ........fd_A.c.f_b

SourceEntity .........f. fd_A

DestinationEntity ......... f_b

Meta-entity ......... FlowDefinition

Meta-attributes

CDIFIdentifier ........fd_A:c

Meta-entity ......... Flow

Meta-attributes

CDIFIdentifier .........f f $\mathrm{c}$ 
Meta-relationship ..... Flow.References.FlowDefinition

Meta-attributes

CDIFIdentifier ........f_c.r.fd_A:c

SourceEntity ........... f $\mathrm{f}_{\mathrm{f}}$

DestinationEntity ...... fd_A:c

Meta-relationship ..... FlowDefinition.Contains.Flow

Meta-attributes

CDIFIdentifier ......... fd_A.c.f_c

SourceEntity .......... fd_A

DestinationEntity ......... f_c

Meta-entity .......... Flow

Meta-attributes

CDIFIdentifier .......... f_B

Meta-relationship ..... Flow.References.FlowDefinition

Meta-attributes

CDIFIdentifier ........f_B.r.fd_A:b

SourceEntity ............. $f_{-} B$

DestinationEntity $\ldots \ldots \ldots$ fd_A:b

Meta-entity ......... Flow

Meta-attributes

CDIFIdentifier .......... f_C

Name ............. "C"

Meta-relationship ..... Flow.References.FlowDefinition

Meta-attributes

CDIFIdentifier $\ldots \ldots \ldots$ f_C.r.fd_A:c

SourceEntity .......... f_C

DestinationEntity $\ldots \ldots \ldots$ fd_A:c

Meta-relationship .... . FlowProducerConsumer.Produces.Flow

Meta-attributes

CDIFIdentifier ......... f_A.p.f_B

SourceEntity ............ f_A

DestinationEntity .......f_B

Meta-relationship ..... FlowProducerConsumer.Produces.Flow

Meta-attributes

CDIFIdentifier ......... f_A.p.f_C

SourceEntity ............A

DestinationEntity .......... f_C 
Meta-relationship ..... FlowProducerConsumer.Consumes.Flow

Meta-attributes

CDIFIdentifier ........f_B.c.f_A

SourceEntity ........... f_B

DestinationEntity ......... f_A

Meta-relationship ..... FlowProducerConsumer.Consumes.Flow

Meta-attributes

CDIFIdentifier .........f_C.c.f_A

SourceEntity .......... f_C

DestinationEntity ........f_A

1* This is needed to show semantically that the flow B is a part of the flow A, and that it's the part $b$ which is contained by the FlowDefinition fd_A. We show the same semantics for the flow C.*I

Meta-entity ......... EquivalenceSet

Meta-attributes

CDIFIdentifier ........es_b

Meta-relationship ..... EquivalenceSet.HasMember.Flow

Meta-attributes

CDIFIdentifier .........es_b.hm.f_B

SourceEntity ..........es_b

DestinationEntity ......... f_B

Meta-entity ......... ReferencedElement

Meta-attributes

CDIFIdentifier .........re_b

Meta-relationship ...... EquivalenceSet.HasMember.ReferencedElement

Meta-attributes

CDIFIdentifier ........es_b.hm.re_b

SourceEntity .......... es_b

DestinationEntity ....... re_b

Meta-relationship .... . ReferencedElement.DefinesPath.Flow

Meta-attributes

CDIFIdentifier ........ re_b.dp.f_A

SourceEntity .......... re_b

DestinationEntity ....... f_A

SequenceNumber ........ 1 
Meta-relationship ..... ReferencedElement.DefinesPath.Flow

Meta-attributes

CDIFIdentifier ........ re_b.dp.f_b

SourceEntity .........rerb

DestinationEntity ........f_b

SequenceNumber .......2

Meta-entity ......... EquivalenceSet

Meta-attributes

CDIFIdentifier ........es_c

Meta-relationship ..... EquivalenceSet.HasMember.Flow

Meta-attributes

CDIFIdentifier ........es_c.hm.f_C

SourceEntity ..........e. es_c

DestinationEntity ......... f_C

Meta-entity ......... ReferencedElement

Meta-attributes

CDIFIdentifier .........re.c

Meta-relationship ..... EquivalenceSet.HasMember.ReferencedElement

Meta-attributes

CDIFIdentifier .........es_c.hm.re_c

SourceEntity ..........e. es_c

DestinationEntity ........re_c

Meta-relationship ..... ReferencedElement.DefinesPath.Flow

Meta-attributes

CDIFIdentifier $\ldots$. m re.re.dp.f_A

SourceEntity ..........re_c

DestinationEntity ....... f_A

SequenceNumber ....... 1

Meta-relationship ..... ReferencedElement.DefinesPath.Flow

Meta-attributes

CDIFIdentifier .........re_c.dp.f_c

SourceEntity .......... re_c

DestinationEntity ........f_c

SequenceNumber $\ldots \ldots \ldots 2$ 


\subsubsection{IDEF0 Joins}

IDEF0 Joins can be also categorized into similar to IDEF0 Forks categories:

- "A Copy" Join

- "A Portion" Join

- "The Sum" Join

\subsubsection{1 "A Copy" Join}

\section{IDEF0 Join}

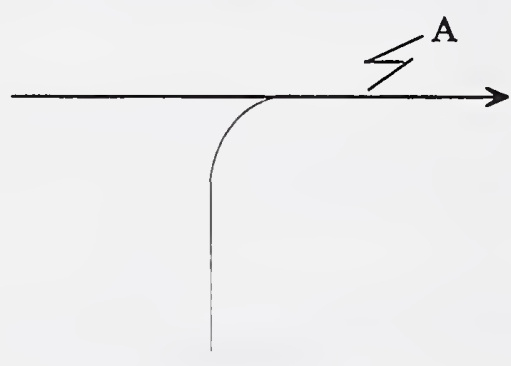

\section{Interpretation}

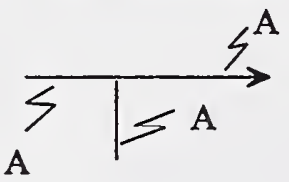

CDIF Flow Merge

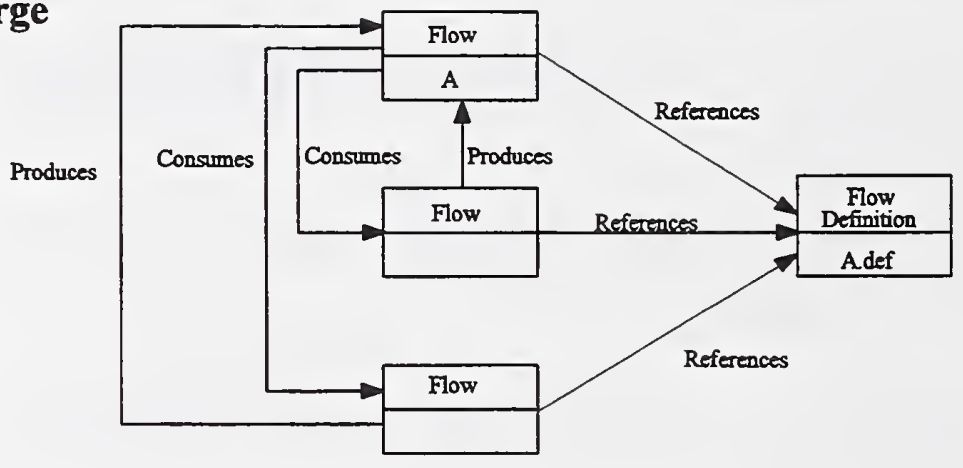

Figure 7. "A Copy" Join 
The diagram above shows the "Copy" type of IDEF0 Join and its mapping into the CDIF graphical representation of the transfer format. The mapping is very similar to the mapping of the IDEF0 "A Copy" Fork. The only difference is in role players for consumers and producers. In the "Copy" Fork, Flow A produces Flows (copies of A), whereas in the "Copy" Join, Flow A consumes Flows (copies of A). Also, in the "Copy" Fork, Flows (copies of A) consume Flow A, whereas in the "Copy" Join, Flows (copies of A) produce Flow A. The following is the CDIF mapping (graphically) of the example above.

Meta-entity FlowDefinition

Meta-attributes

CDIFIdentifier .........fd_A

Name "A.def"

Meta-entity Flow

Meta-attributes

CDIFIdentifier ........... $\mathrm{f} \_\mathrm{A}$

Name ............." $\mathrm{A} "$

Meta-relationship ..... Flow.References.FlowDefinition

Meta-attributes

CDIFIdentifier .........f_A.r.fd_A

SourceEntity ........... f_A

DestinationEntity .......fd_A

Meta-entity Flow

Meta-attributes

CDIFIdentifier .........f_A'

Meta-relationship ...... Flow.References.FlowDefinition

Meta-attributes

CDIFIdentifier ........f.A'.r.fd_A

SourceEntity ..............'

DestinationEntity ........fd_A

Meta-entity ......... Flow

Meta-attributes

CDIFIdentifier .........f_A" 
Meta-relationship ..... Flow.References.FlowDefinition

Meta-attributes

CDIFIdentifier ........f.f".r.fd_A

SourceEntity ..........f.

DestinationEntity .......fd_A

/* The following part of the mapping is in Bold to emphasize the difference between the mapping of an IDEF0 Fork and a Join. */

Meta-relationship .... . FlowProducerConsumer.Produces.Flow

Meta-attributes

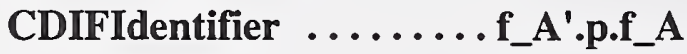

SourceEntity .................

DestinationEntity ........ f_A

Meta-relationship ... . . FlowProducerConsumer.Produces.Flow

Meta-attributes

CDIFIdentifier .........f_A".p.f_A

SourceEntity ..............A"

DestinationEntity ........ f_A

Meta-relationship ... . . FlowProducerConsumer.Consumes.Flow

Meta-attributes

CDIFIdentifier ........ f_A.c.f_A'

SourceEntity ..............A

DestinationEntity ........ f_A'

Meta-relationship .... . FlowProducerConsumer.Consumes.Flow

Meta-attributes

CDIFIdentifier ........f_A.c.f_A"

SourceEntity ............. f_A

DestinationEntity ....... f_A" 


\subsubsection{2 "A Portion" Join}

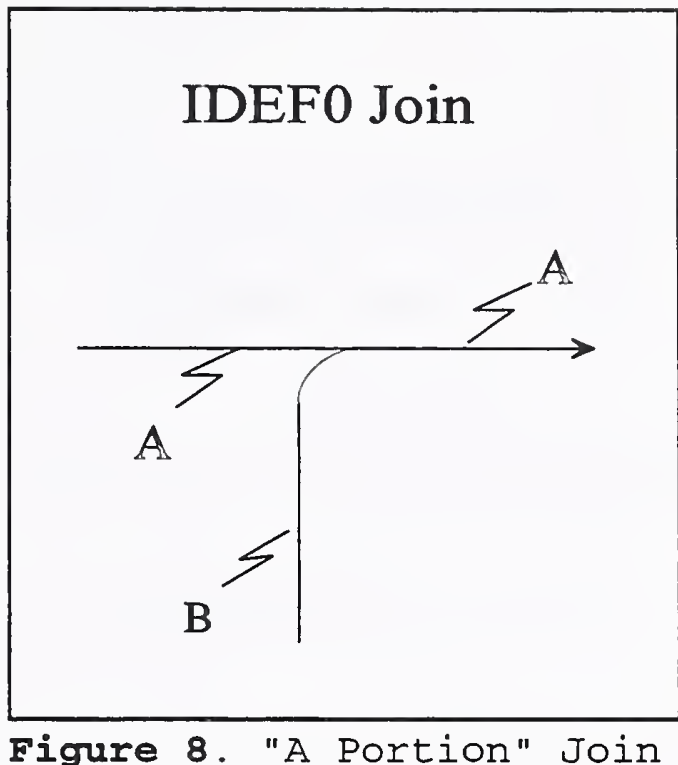

The above figure is another type of IDEF0 Join. The interpretation for this type of IDEF0 Join is the following: flow A consists of flow B (and possibly something else); more of B flows into A. A graphical mapping of this type of fork can be found in Appendix A. Again, the mapping for "A Portion" Join can be obtained from the mapping of the "A Portion" Fork by flipping the CDIF meta-relationship FlowProducerConsumer.Consumes.Flow with FlowProducerConsumer.Produces.Flow, and vise versa. The following is the CDIF transfer file for the Figure 8.

Meta-entity ......... FlowDefinition

Meta-attributes

CDIFIdentifier .........fd_A

Name ..............."A.def"

Meta-entity ......... Flow

Meta-attributes

CDIFIdentifier ........... f_A

Name ............." $\mathrm{A}^{\prime}$

Meta-relationship ..... . Flow.References.FlowDefinition

Meta-attributes

CDIFIdentifier .........f_A.r.fd_A

SourceEntity ..............A

DestinationEntity .......fd_A 
Meta-entity ......... FlowDefinition

Meta-attributes

CDIFIdentifier $\ldots \ldots \ldots$ fd_A:b

Meta-entity ......... Flow

Meta-attributes

CDIFIdentifier ........f f b

Meta-relationship ..... Flow.References.FlowDefinition

Meta-attributes

CDIFIdentifier ........f_b.r.fd_A:b

SourceEntity ......... f $\_b$

DestinationEntity $\ldots \ldots$ fd_A:b

Meta-relationship ...... FlowDefinition.Contains.Flow

Meta-attributes

CDIFIdentifier ........fd_A.c.f_b

SourceEntity ..........fd_A

DestinationEntity ........ f_b

Meta-entity ......... Flow

Meta-attributes

CDIFIdentifier .........f.'

Meta-relationship ...... Flow.References.FlowDefinition

Meta-attributes

CDIFIdentifier ........f_A'.r.fd_A

SourceEntity ..........f_A'

DestinationEntity ........fd_A

Meta-entity ......... Flow

Meta-attributes

CDIFIdentifier .......... f_B

Meta-relationship .... . . Flow.References.FlowDefinition

Meta-attributes

CDIFIdentifier .......f.B.r.fd_A:b

SourceEntity .......... f_B

DestinationEntity $\ldots \ldots \ldots$ fd_A:b 
Meta-relationship .... . FlowProducerConsumer.Produces.Flow

Meta-attributes

CDIFIdentifier ........f_.A'.p.f_A

SourceEntity .................

DestinationEntity ........ f_A

Meta-relationship .... . FlowProducerConsumer.Produces.Flow

Meta-attributes

CDIFIdentifier ........f_B.p.f_A

SourceEntity .......... f_B

DestinationEntity ......... f_A

Meta-relationship .... . FlowProducerConsumer.Consumes.Flow

Meta-attributes

CDIFIdentifier ........f_A.c.f_A'

SourceEntity ............A

DestinationEntity ....... f f $A^{\prime}$

Meta-relationship ..... FlowProducerConsumer.Consumes.Flow

Meta-attributes

CDIFIdentifier ..........._A.c.f_B

SourceEntity .............A

DestinationEntity ....... f_B

$1 *$ This is needed to show semantically that the flow B is a part of the flow A, and that it's the part $\mathrm{b}$ which is contained by the FlowDefinition fd_A. */

Meta-entity ......... EquivalenceSet

Meta-attributes

CDIFIdentifier $\ldots . .$. . es_b

Meta-relationship ..... EquivalenceSet.HasMember.Flow

Meta-attributes

CDIFIdentifier ........es_b.hm.f_B

SourceEntity ........... es_b

DestinationEntity ......... f_B

Meta-entity ........ ReferencedElement

Meta-attributes

CDIFIdentifier .........re_b 
Meta-relationship ..... EquivalenceSet.HasMember.ReferencedElement

Meta-attributes

CDIFIdentifier .........es_b.hm.re_b

SourceEntity .......... es_b

DestinationEntity .......re_b

Meta-relationship ..... ReferencedElement.DefinesPath.Flow

Meta-attributes

CDIFIdentifier $\ldots \ldots$. . re_b.dp.f_A

SourceEntity .......... re_b

DestinationEntity ......... f_A

SequenceNumber ........

Meta-relationship ..... ReferencedElement.DefinesPath.Flow

Meta-attributes

CDIFIdentifier $\ldots \ldots$. . re_b.dp.f_b

SourceEntity ..........re_b

DestinationEntity ........ $\mathrm{f}_{-} \mathrm{b}$

SequenceNumber .......2

\subsubsection{3 "The Sum" Join}

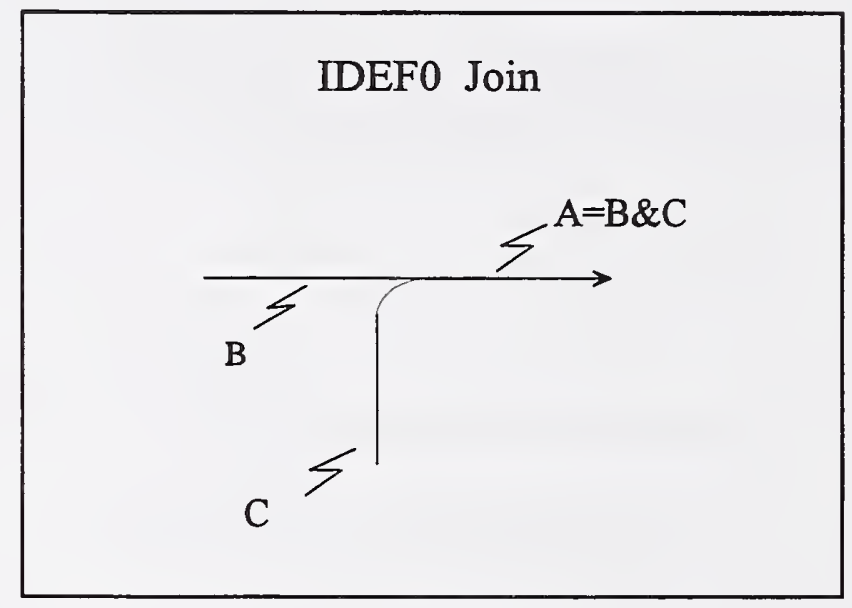

Figure 9. "The Sum" Join

The above figure is the third type of IDEF0 Join. The flow A contains flows B and C in its structure. A graphical representation of the mapping of this type of fork can be found in Appendix A. Again, like in the previous types of Join, by flipping Consumes and Produces we can transform "The Sum" Fork mapping into "The Sum" Join mapping The following is the CDIF transfer file for the Figure 9. 
Meta-entity ......... FlowDefinition

Meta-attributes

CDIFIdentifier .........fd_A

Name

Meta-entity .......... Flow

Meta-attributes

CDIFIdentifier .........f_A

Name ............. "A"

Meta-relationship ..... . Flow.References.FlowDefinition

Meta-attributes

CDIFIdentifier .........f_A.r.fd_A

SourceEntity ............ f_A

DestinationEntity ........ fd_A

Meta-entity ......... FlowDefinition

Meta-attributes

CDIFIdentifier ........fd_A:b

Meta-entity ......... Flow

Meta-attributes

CDIFIdentifier ..........f $\mathrm{f} b$

Meta-relationship ..... Flow.References.FlowDefinition

Meta-attributes

CDIFIdentifier ........f_b.r.fd_A:b

SourceEntity .......... f $\_b$

DestinationEntity $\ldots \ldots \ldots$ fd_A:b

Meta-relationship ..... . FlowDefinition.Contains.Flow

Meta-attributes

CDIFIdentifier ........fd_A.c.f_b

SourceEntity .........fd_A

DestinationEntity ........ f_b

Meta-entity ......... FlowDefinition

Meta-attributes

CDIFIdentifier ........fd_A:c

Meta-entity ......... Flow

Meta-attributes

CDIFIdentifier ..........f $f_{-}$ 
Meta-relationship ...... Flow.References.FlowDefinition

Meta-attributes

CDIFIdentifier ........f_c.r.fd_A:c

SourceEntity ............ $f_{-} \mathrm{c}$

DestinationEntity .......fd_A:c

Meta-relationship ..... FlowDefinition.Contains.Flow

Meta-attributes

CDIFIdentifier ........fd_A.c.f_c

SourceEntity .........fd_A

DestinationEntity ........f_c

Meta-entity ......... Flow

Meta-attributes

CDIFIdentifier ........f_B

Meta-relationship ..... Flow.References.FlowDefinition

Meta-attributes

CDIFIdentifier ........f_B.r.fd_A:b

SourceEntity ......... f_B

DestinationEntity $\ldots \ldots \ldots$ fd_A:b

Meta-entity ......... Flow

Meta-attributes

CDIFIdentifier .......... $\mathrm{f} C$

Name ............ "C"

Meta-relationship ..... . Flow.References.FlowDefinition

Meta-attributes

CDIFIdentifier .......f.C.r.fd_A:c

SourceEntity .......... f_C

DestinationEntity $\ldots \ldots \ldots$ fd_A:c

Meta-relationship .... . FlowProducerConsumer.Consumes.Flow Meta-attributes

CDIFIdentifier ........f_A.p.f_B

SourceEntity ...........A

DestinationEntity ........ f_B

Meta-relationship .... . FlowProducerConsumer.Consumes.Flow Meta-attributes

CDIFIdentifier ...........A.p.f_C

SourceEntity .............A

DestinationEntity ........ f_C 
Meta-relationship .... . FlowProducerConsumer.Produces.Flow

Meta-attributes

CDIFIdentifier ........f_B.c.f_A

SourceEntity ............ $\mathbf{f}_{-}$B

DestinationEntity ........ f_A

Meta-relationship .... . FlowProducerConsumer.Produces.Flow

Meta-attributes

CDIFIdentifier .........f_C.c.f_A

SourceEntity ............

DestinationEntity ...........A

/* This is needed to show semantically that the flow B is a part of the flow A, and that it's the part $b$ which is contained by the FlowDefinition fd_A. We show the same semantics for the flow C.*I

Meta-entity ......... EquivalenceSet

Meta-attributes

CDIFIdentifier ........es_b

Meta-relationship ...... EquivalenceSet.HasMember.Flow

Meta-attributes

CDIFIdentifier ........es_b.hm.f_B

SourceEntity ..........es_b

DestinationEntity .......... f_B

Meta-entity ........ ReferencedElement

Meta-attributes

CDIFIdentifier ........re_b

Meta-relationship ..... EquivalenceSet.HasMember.ReferencedElement

Meta-attributes

CDIFIdentifier .......e.es_b.hm.re_b

SourceEntity ........... es_b

DestinationEntity $\ldots \ldots \ldots$ re_b

Meta-relationship ..... ReferencedElement.DefinesPath.Flow

Meta-attributes

CDIFIdentifier $\ldots \ldots$. mere_b.dp.f_A

SourceEntity .......... re_b

DestinationEntity ............A

SequenceNumber ....... 1 
Meta-relationship ..... ReferencedElement.DefinesPath.Flow

Meta-attributes

CDIFIdentifier ........ re_b.dp.f_b

SourceEntity .......... re_b

DestinationEntity ......... f_b

SequenceNumber .......2

Meta-entity ......... EquivalenceSet

Meta-attributes

CDIFIdentifier .........es_c

Meta-relationship ...... EquivalenceSet.HasMember.Flow

Meta-attributes

CDIFIdentifier ........es_c.hm.f_C

SourceEntity ..........e. es_c

DestinationEntity .......... f_C

Meta-entity ......... ReferencedElement

Meta-attributes

CDIFIdentifier $\ldots . . . \cdots$ re_c

Meta-relationship ...... EquivalenceSet.HasMember.ReferencedElement

Meta-attributes

CDIFIdentifier ........e. es_c.hm.re_c

SourceEntity ...........es_c

DestinationEntity $\ldots . .$. re_c

Meta-relationship ..... ReferencedElement.DefinesPath.Flow

Meta-attributes

CDIFIdentifier ......... re_c.dp.f_A

SourceEntity .......... re_c

DestinationEntity ...........A

SequenceNumber .......

Meta-relationship ..... ReferencedElement.DefinesPath.Flow

Meta-attributes

CDIFIdentifier ........ re_c.dp.f_c

SourceEntity ...........re_c

DestinationEntity ........f_c

SequenceNumber ......2 


\subsubsection{IDEF0 Calls}

Like with the IDEF0 Forks and Joins, we can categorize IDEF0 Calls into four groups:

- "Another Data Model"

- "A Document"

- Split By Type

- A Drop in the Level of Abstraction

\subsubsection{1 "Another Data Model" Call}

The following figure shows a situation when the calls refer to another data model. An instance of DFMProcessDefinition for that data model had to be created (while mapping that data model into CDIF). Reference all the DFMProcesses (processes) which have a call to that data model to the DFMProcessDefinition for that data model. The processes A01and A0211 (see Figure 10) both have calls to the stand-alone data model B-0. The DFMProcessDefinition B-0.def had to be originally created for the data model B-0. In the current data model, DFMProcesses A01 and A0211have to reference that same DFMProcessDefinition to reuse the structure and definition of the data model B-0.

\section{IDEFO Call}
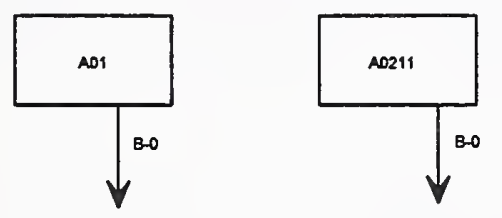

$B-0$

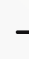

\section{CDIF}

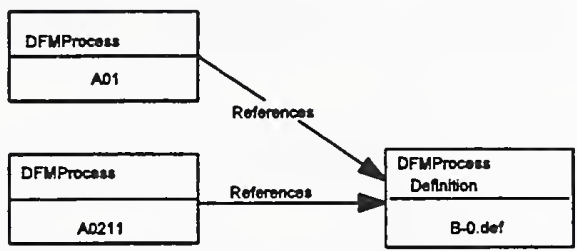

Figure 10. "Another Data Model" Call 
The following is the CDIF mapping of the "Another Data Model" Call above.

/* The following was defined for the data model B-0. */

Meta-entity ......... DataFlowModel

Meta-attributes

CDIFIdentifier ........... call1_example

Name .............. Example"

Meta-entity ......... DFMProcessDefinition

Meta-attributes

CDIFIdentifier $\ldots \ldots \ldots$. pd_B-0

Name ............."B-0_def"

Meta-relationship ..... . DataFlowModel.HasRoot.DFMProcessDefinition

Meta-attributes

CDIFIdentifier ........... call1_example.hr.pd_B-0

SourceEntity ............ call1_example

DestinationEntity ........pd_B-0

$1 *$ The following should be difined for the current mapping of the processes A01 and A0211.*/

Meta-entity ......... DFMProcess

Meta-attributes

CDIFIdentifier .........dfmp_A01

Name ............. "A01"

Meta-relationship ...... DFMProcess.References.DFMProcessDefinition

Meta-attributes

CDIFIdentifier $\ldots \ldots \ldots$ dfmp_A01.r.pd_B-0

SourceEntity ..........dfmp_A01

DestinationEntity $\ldots \ldots \ldots$ pd_B-0

Meta-entity ......... DFMProcess

Meta-attributes

CDIFIdentifier .........dfmp_A0211

Name ............. "A0211" 
Meta-relationship ..... . DFMProcess.References.DFMProcessDefinition

Meta-attributes

CDIFIdentifier $\ldots \ldots \ldots$. dfmp_A0211.r.pd_B-0

SourceEntity .......... dfmp_A0211

DestinationEntity $\ldots \ldots \ldots$. pd_B-0

\subsubsection{2 "A Document" Call}

This is another type of IDEF0 Call. This call is simply a reference to a book, an article, or a document. In this case, a DFMProcess that has this type of call should have a pointer (usually the same content as in IDEF0 call) to the document in its local meta-attribute BriefDescription and/or FullDescription.

The following is an example of "A Document" Call. The process A01 references the document FIPS 184 (see Figure 12).

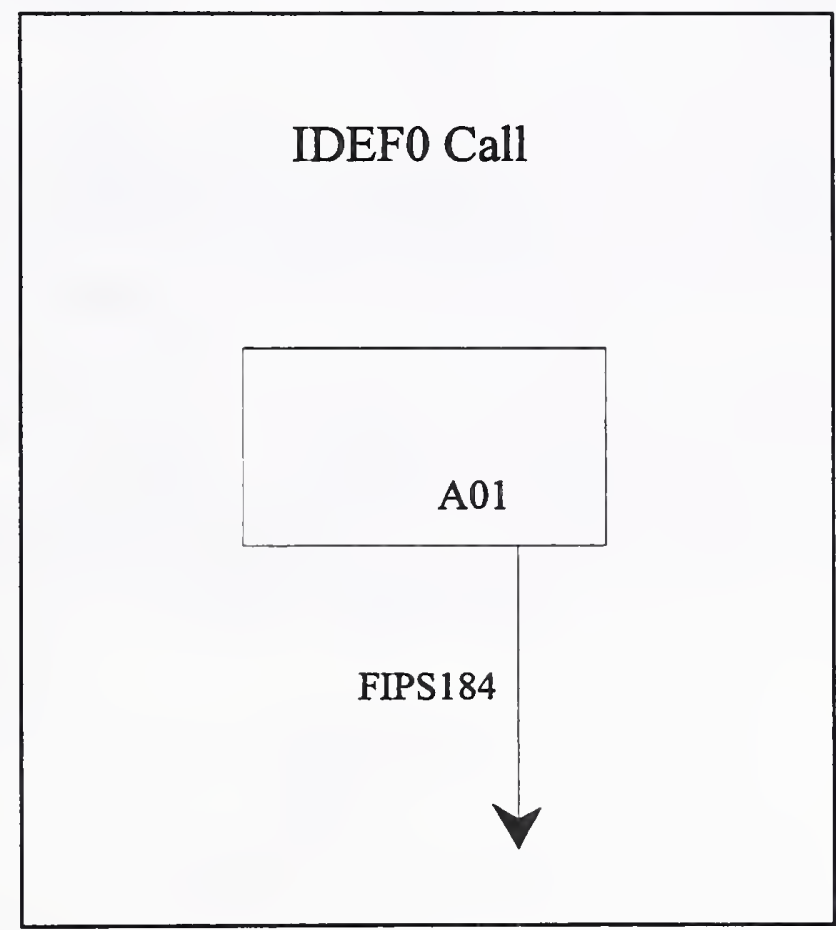

Figure 11. "A Document" Call

The following is the CDIF mapping of the call above. 


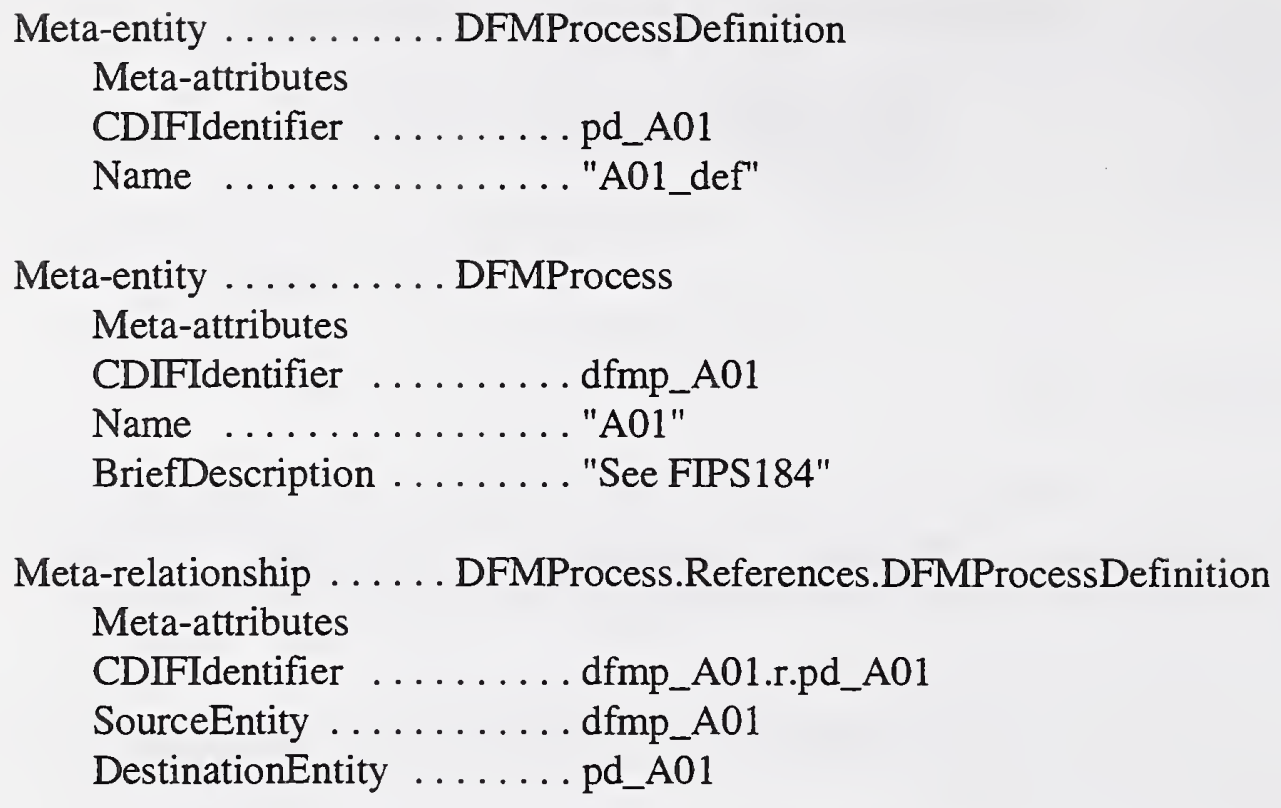

\subsubsection{SBT (Split By Type) Call}

This is the third type of IDEF0 Call. A single box (process, function) in a decomposition calls one from a set of many activities at a lower level of abstraction. The activities at the lower level are of a certain type. For example, Mechanical Process Model, Bending Process Model, and Chemical Treating Process Model are all types of process models.

The following is an example of a SBT Call ${ }^{3}$. The process A01 will either call Mechanical Process Model, Bending Process Model, or Chemical Treating Process Model. The structure of SBT Call can be captured by the CDIF meta-relationship DFMProcessDefinition.Contains.DFMProcess (an instance for each member of the set). Also, the meta-attribute Operator of the meta-entity DFMProcessDefinition shall be set to $X O R$.

3 This example was taken from Clarence Feldmann's article on calls and mechanisms. 


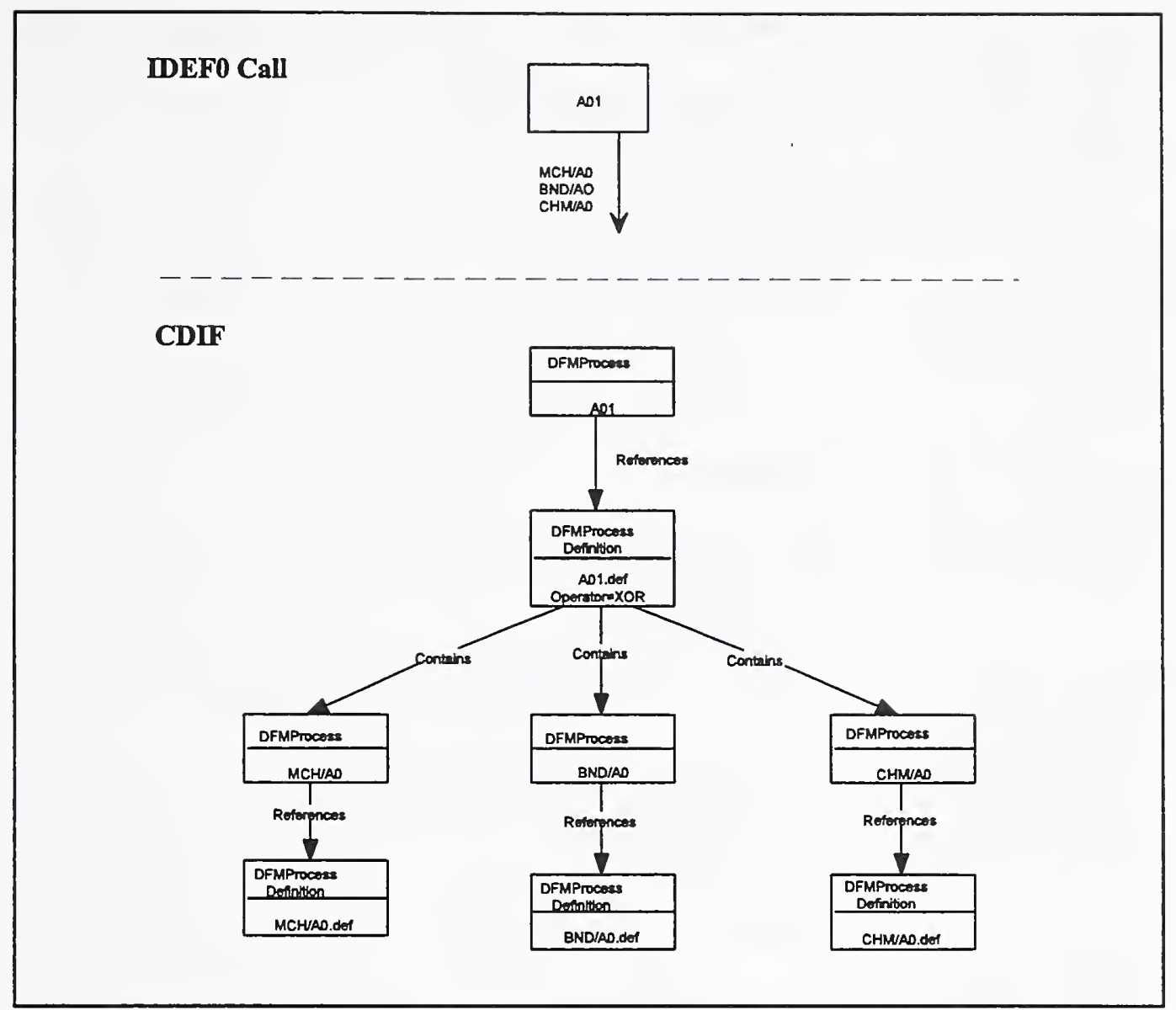

Figure 12. SBT Call

The following is the CDIF mapping of the call above.

Meta-entity ........ DFMProcessDefinition

Meta-attributes

CDIFIdentifier ......... pd_A01

Name ............ A01_def"

Operator ...........XOR

Meta-entity ......... DFMProcess

Meta-attributes

CDIFIdentifier .........dfmp_A01

Name ............. "A01" 
Meta-relationship ..... DFMProcess.References.DFMProcessDefinition

Meta-attributes

CDIFIdentifier ........dfmp_A01.r.pd_A01

SourceEntity ......... dfmp_A01

DestinationEntity ........pd_A01

Meta-entity ........ DFMProcessDefinition

Meta-attributes

CDIFIdentifier .........pd_MCH/A0

Name ............"MCH/A0_def"

Meta-entity ......... DFMProcess

Meta-attributes

CDIFIdentifier ........ dfmp_MCH/A0

Name ........... "MCH/A0"

Meta-relationship ..... DFMProcess.References.DFMProcessDefinition

Meta-attributes

CDIFIdentifier ........dfmp_MCH/A0.r.pd_MCH/A0

SourceEntity .......... dfmp_MCH/A0

DestinationEntity .......pd_MCH/A0

Meta-entity ........ DFMProcessDefinition

Meta-attributes

CDIFIdentifier $\ldots \ldots \ldots$. pd_BND/A0

Name ............"BND/A0_def"

Meta-entity ......... DFMProcess

Meta-attributes

CDIFIdentifier ........dfmp_BND/A0

Name $\ldots \ldots \ldots \ldots \ldots$. BND/A0"

Meta-relationship ..... DFMProcess.References.DFMProcessDefinition

Meta-attributes

CDIFIdentifier ........dfmp_BND/A0.r.pd_BND/A0

SourceEntity .........dfmp_BND/A0

DestinationEntity $\ldots \ldots \ldots$ pd_BND/A0

Meta-entity ......... DFMProcessDefinition

Meta-attributes

CDIFIdentifier .......... pd_CHM/A0

Name ............ "CHM/A0_def" 
Meta-entity DFMProcess

Meta-attributes

CDIFIdentifier .........dfmp_CHM/A0

Name "CHM/A0"

Meta-relationship ...... DFMProcess.References.DFMProcessDefinition Meta-attributes

CDIFIdentifier ........dfmp_CHM/A0.r.pd_CHM/A0

SourceEntity ..........dfmp_CHM/A0

DestinationEntity pd_CHM/AO

I* This is needed to show the structure of the DFMProcessDefinition A01_def. */

Meta-relationship ...... DFMProcessDefinition.Contains.DFMProcess

Meta-attributes

CDIFIdentifier $\ldots \ldots \ldots$ pd_A01.c.dfmp_MCH/A0

SourceEntity ............pd_A01

DestinationEntity .......dfmp_MCH/A0

Meta-relationship ..... DFMProcessDefinition.Contains.DFMProcess

Meta-attributes

CDIFIdentifier .........pd_A01.c.dfmp_BND/A0

SourceEntity ...........pd_A01

DestinationEntity ....... dfmp_BND/A0

Meta-relationship ...... DFMProcessDefinition.Contains.DFMProcess

Meta-attributes

CDIFIdentifier $\ldots \ldots \ldots$. pd_A01.c.dfmp_CHM/A0

SourceEntity .......... pd_A01

DestinationEntity ......dfmp_CHM/A0 


\subsubsection{A Drop in the Level of Abstraction Call}

\section{IDEF0 Call}

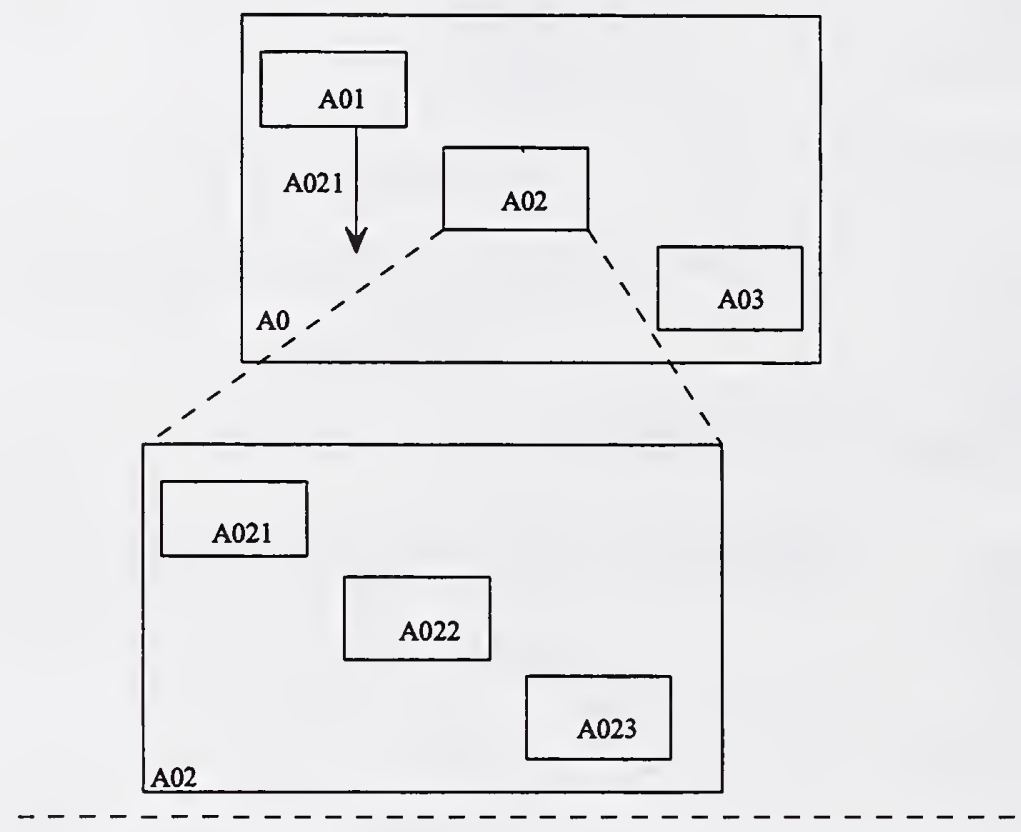

\section{$\mathrm{CDIF}$}

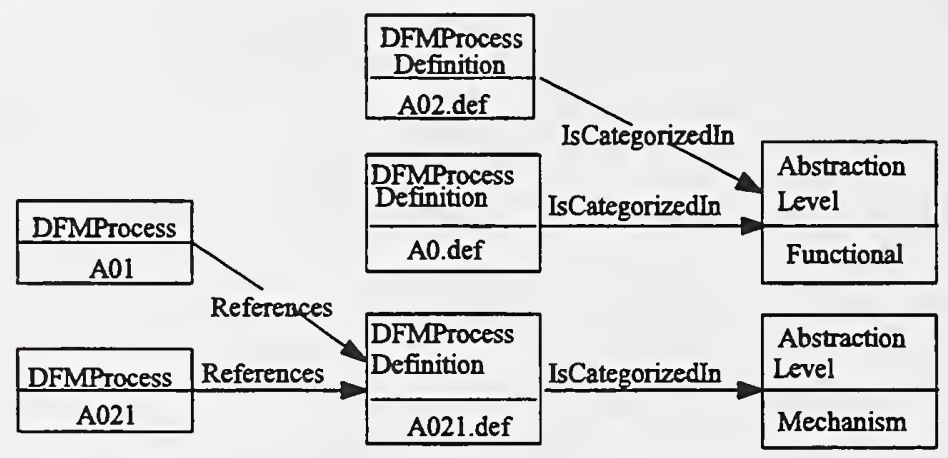

Figure 13. A Drop in the Level of Abstraction Call

This is the fourth type of IDEF0 Call. The figure above demonstrates the mapping of this Call, which indicates a drop in the Level of Abstraction (from the functional level to the mechanism level). The functions A01 and A021are at different levels of abstraction, and the call provides a link between these levels. 
The following is the CDIF mapping of the above call. A couple of new CDIF objects are introduced. By the means of the meta-entity AbstractionLevel and the meta-relationship DFMProcessDefinition.IsCategorizedIn.AbstractionLevel we are able to provide that link between the Functional level and Mechanism level of abstraction.

Meta-entity ......... DFMProcessDefinition

Meta-attributes

CDIFIdentifier .........pd_A0

Name ........... A0_def"

Meta-entity ........ DFMProcessDefinition

Meta-attributes

CDIFIdentifier .........pd_A02

Name ............."A02_def"

Meta-entity ......... AbstractionLevel

Meta-attributes

CDIFIdentifier ..........al_Functional

Name ............ "Functional"

Meta-relationship . . . . . DFMProcessDefinition.IsCategorizedIn.AbstractionLevel

Meta-attributes

CDIFIdentifier $\ldots \ldots \ldots$ pd_A0.ici.al_Functional

SourceEntity $\ldots \ldots \ldots \ldots$ pd_A0

DestinationEntity ........ al_Functional

Meta-relationship . . . . . DFMProcessDefinition.IsCategorizedIn.AbstractionLevel

Meta-attributes

CDIFIdentifier .........pd_A02.ici.al_Functional

SourceEntity ............pd_A02

DestinationEntity ........ al_Functional

Meta-entity ......... DFMProcessDefinition

Meta-attributes

CDIFIdentifier .........pd_A021

Name

$$
\text { "A021_def" }
$$

Meta-entity

DFMProcess

Meta-attributes

CDIFIdentifier .........dfmp_A01

Name

"A01" 
Meta-relationship ..... . DFMProcess.References.DFMProcessDefinition

Meta-attributes

CDIFIdentifier .........dfmp_A01.r.pd_A021

SourceEntity ..........dfmp_A01

DestinationEntity ........pd_A021

Meta-entity ......... DFMProcess

Meta-attributes

CDIFIdentifier .........dfmp_A021

Name ............."A021"

Meta-relationship ..... DFMProcess.References.DFMProcessDefinition

Meta-attributes

CDIFIdentifier .........dfmp_A021.r.pd_A021

SourceEntity ...........dfmp_A021

DestinationEntity $\ldots . .$. . pd_A021

Meta-entity ......... AbstractionLevel

Meta-attributes

CDIFIdentifier .........al_Mechanism

Name ..............."Mechanism"

Meta-relationship ..... DFMProcessDefinition.IsCategorizedIn.AbstractionLevel Meta-attributes

CDIFIdentifier .........pd_A01.ici.al_Mechanism

SourceEntity ............pd_A01

DestinationEntity .........al_Mechanism

Meta-relationship ..... . DFMProcessDefinition.IsCategorizedIn.AbstractionLevel Meta-attributes

CDIFIdentifier ........pd_A021.ici.al_Mechanism

SourceEntity $\ldots \ldots \ldots \ldots$. pd_A021

DestinationEntity .........al_Mechanism 


\subsection{Process/Flow Decomposition}

We have already mentioned Process Decomposition in section 4.1 .5 (pg. $\mathrm{x}$ ). In this section we will take a look at six different types of combinations of Process Decomposition with Flow Decomposition.

Process Decomposition is the same for all the combinations: process $\mathrm{A} 0$ decomposes into processes A01, A02, and A03. The difference is in Flow Decomposition.

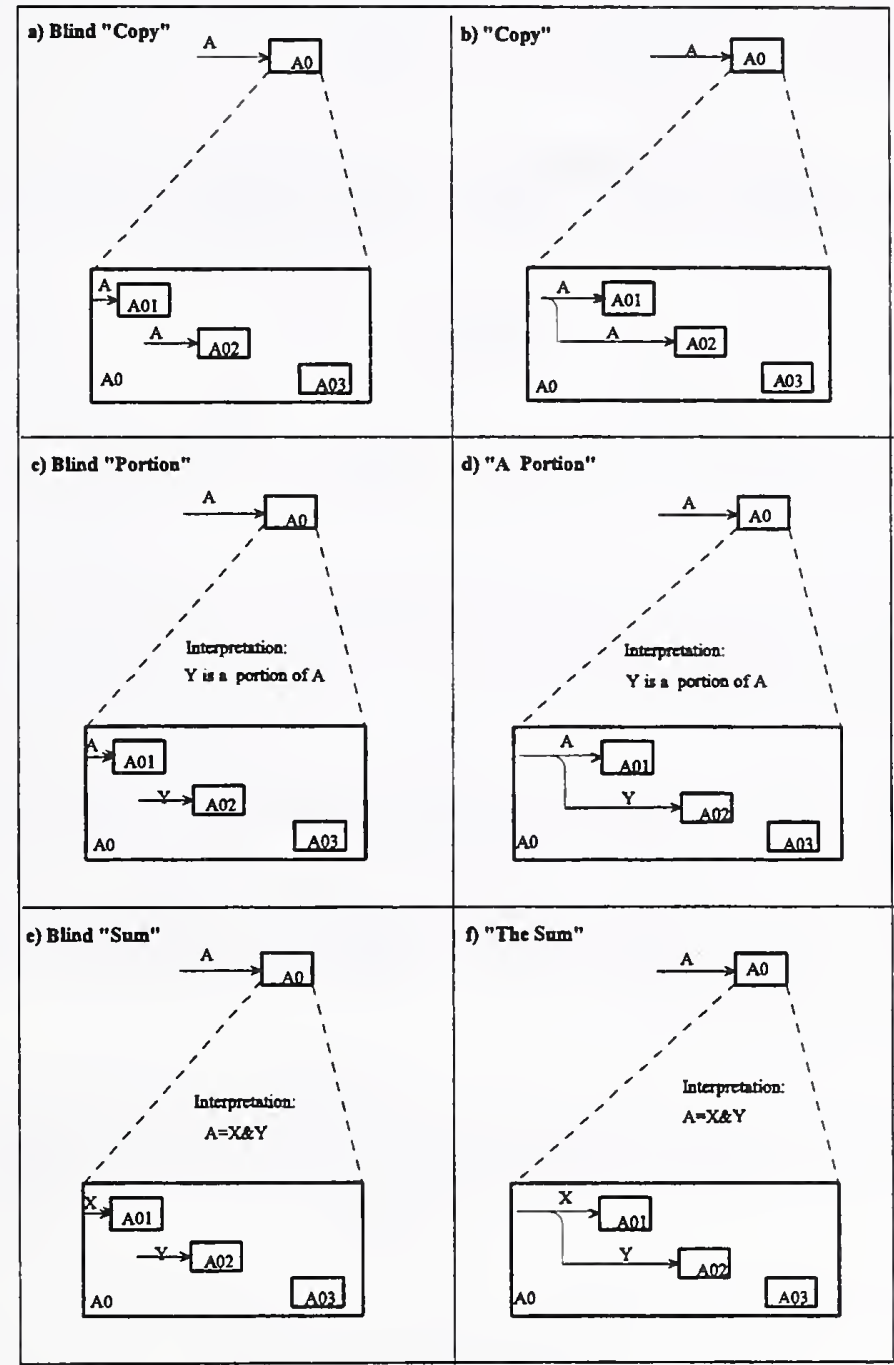

Figure 14. Types of Flow Decomposition 
In all the cases, an input A flows into the process (function) A0 (see Figure 14). The only difference is how this flow A decomposes on the lower level of abstraction. As with IDEF0 Forks and Joins, a similar argument about Fork/Join types can be applied to a flow decomposition. We can look at a flow decomposition as a simple Fork (or Join) combined with a process decomposition.

Like with Forks and Joins, we have the "Copy" type of a flow decomposition: the input A gets "copied", and the flow A and its copy are the inputs for A01and A02 on the lower level of abstraction (fig. 14.b). The difference between the figure 14.a and 14.b is how (or where) input A gets "copied." On the figure 14.a, we do not see that A gets "copied", but we assume so. On the figure 14.b, we do see the split (or "A Copy" in this case) of the flow A from the higher level into two flows. That is why the title of the figure 14.a is "Blind Copy", and the title of the figure 14.b is just "A Copy."

Similar argument can be applied to the figures 14.c and 14.d, and figures 14.e and 14.f. The following are the six different types of Flow Decomposition:

- "Blind Copy"

- "A Copy"

- "Blind Portion"

- "A Portion"

- "Blind Sum"

- "The Sum"

You can find graphical representations for the CDIF mappings of all these types of Flow Decomposition in the Appendix A.

Note:

One of the mapping rules hold the following: If there exists a relationship

FlowProducerConsumer.Produces.Flow, where FlowProducerConsumer is a source flow F1, and Flow is a destination flow F2, then there also exists a relationship FlowProducerConsumer.Consumes.Flow, where FlowProducerConsumer is the flow F2, and Flow is the flow F1.

The graphs, however, do not follow this rule because of the limited space on a page. Only one from each pair of the correspondent relationships is shown. 


\subsection{1 "Blind Copy" Flow Decomposition}

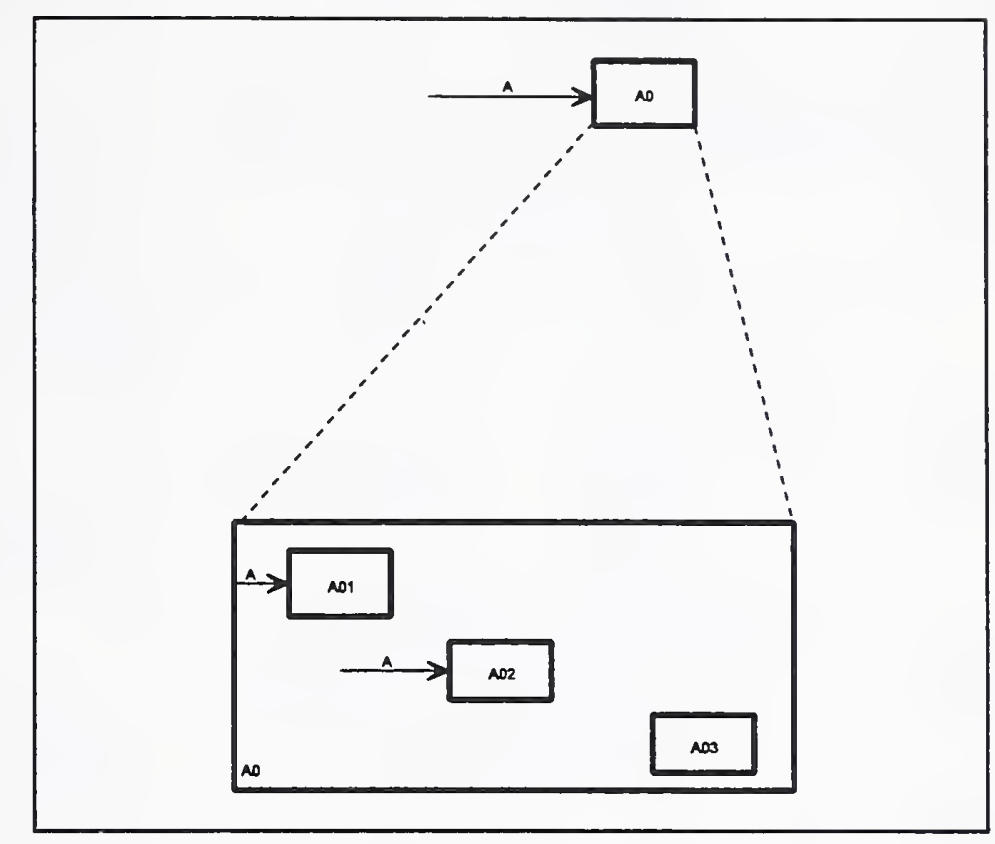

Figure 15. "Blind Copy" Decomposition

The flow A was (blindly) "copied", which means that there are two instances of the same flow (same structure). One instance of flow A is an input to the process A01, and another instance is an input to the process A02. We can reuse the same FlowDefinition for all the instances of flow A because they all have the same structure.

The following is the CDIF mapping of the above decomposition. A graphical representation of the mapping can be found in the Appendix A.

Meta-entity

DFMProcess

Meta-attributes

CDIFIdentifier ..........dfmp_A0

Name "A0"

Meta-entity DFMProcessDefinition

Meta-attributes

CDIFIdentifier $\ldots \ldots \ldots$. pd_A0

Name ............. "A0_def" 
Meta-relationship ..... DFMProcess.References.DFMProcessDefinition

Meta-attributes

CDIFIdentifier $\ldots \ldots \ldots$. dfmp_A0.r.pd_A0

SourceEntity ......... dfmp_A0

DestinationEntity ........pd_A0

Meta-entity .......... Flow

Meta-attributes

CDIFIdentifier ......... f_A

Name ............." A"

Meta-entity ......... FlowInputPort

Meta-attributes

CDIFIdentifier ......... fip_Aa

IsFormal ........... FALSE

Meta-relationship ..... FlowInputPort.Consumes.Flow

Meta-attributes

CDIFIdentifier ......... fip_Aa.c.f_A

SourceEntity .......... fip_Aa

DestinationEntity ........ f_A

Meta-relationship ..... . Flow.Produces.FlowInputPort

Meta-attributes

CDIFIdentifier .........f_A.p.fip_Aa

SourceEntity .......... f_A

DestinationEntity ....... fip_Aa

Meta-entity ......... EquivalenceSet

Meta-attributes

CDIFIdentifier ........es_A

Meta-relationship ... . . EquivalenceSet.HasMember.FlowInputPort

Meta-attributes

CDIFIdentifier $\ldots \ldots \ldots$ es_A.hm.fip_Aa

SourceEntity ........... es_A

DestinationEntity ....... fip_Aa

Meta-entity ........ ReferencedElement

Meta-attributes

CDIFIdentifier .........re_A 
Meta-relationship ...... EquivalenceSet.HasMember.ReferencedElement Meta-attributes

CDIFIdentifier .........es_A.hm.re_A

SourceEntity ........... es_A

DestinationEntity ....... re_A

Meta-relationship ..... ReferencedElement.DefinesPath.ComponentObject

Meta-attributes

CDIFIdentifier $\ldots \ldots \ldots$. re_A.dp.dfmp_A0

SourceEntity .......... re_A

DestinationEntity ......dfmp_A0

SequenceNumber ....... 1

Meta-entity ......... FlowDefinition

Meta-attributes

CDIFIdentifier .........fd_A

Name .............. "A_def"

Meta-relationship ..... Flow.References.FlowDefinition

Meta-attributes

CDIFIdentifier .........f_A.r.fd_A

SourceEntity ............A

DestinationEntity ........fd_A

Meta-relationship ..... . FlowInputPort.References.FlowDefinition

Meta-attributes

CDIFIdentifier ........ fip_Aa.r.fd_A

SourceEntity .......... fip_Aa

DestinationEntity .......fd_A

/* Process Decomposition: the next level of abstraction. */

Meta-entity ........ DFMProcess

Meta-attributes

CDIFIdentifier .........dfmp_A01

Name ............. A01"

Meta-entity ......... DFMProcessDefinition.Contains.DFMProcess

Meta-attributes

CDIFIdentifier .........pd_A0.c.dfmp_A01

SourceEntity .......... pd_A0

DestinationEntity .......dfmp_A01 
Meta-entity ........ DFMProcessDefinition

Meta-attributes

CDIFIdentifier ...........pd_A01

Name ............. A01_def"

Meta-relationship ...... DFMProcess.References.DFMProcessDefinition

Meta-attributes

CDIFIdentifier ........dfmp_A01.r.pd_A01

SourceEntity ..........dfmp_A01

DestinationEntity .........pd_A01

Meta-entity .......... DFMProcess

Meta-attributes

CDIFIdentifier .........dfmp_A02

Name $\ldots \ldots \ldots \ldots \ldots$.A02"

Meta-entity ........ DFMProcessDefinition.Contains.DFMProcess

Meta-attributes

CDIFIdentifier $\ldots \ldots \ldots$. pd_A0.c.dfmp_A02

SourceEntity $\ldots . . \cdots \cdots$. pd_A0

DestinationEntity .......dfmp_A02

Meta-entity ......... DFMProcessDefinition

Meta-attributes

CDIFIdentifier ..............A02

Name ............. "A02_def"

Meta-relationship ...... DFMProcess.References.DFMProcessDefinition

Meta-attributes

CDIFIdentifier ..........dfmp_A02.r.pd_A02

SourceEntity ..........dfmp_A02

DestinationEntity...$\cdots$. pd_A02

Meta-entity ......... DFMProcess

Meta-attributes

CDIFIdentifier ..........dfmp_A03

Name ............. "A03"

Meta-entity ......... DFMProcessDefinition.Contains.DFMProcess

Meta-attributes

CDIFIdentifier ..........pd_A0.c.dfmp_A03

SourceEntity ...........pd_A0

DestinationEntity ........dfmp_A03 
Meta-entity DFMProcessDefinition

Meta-attributes

CDIFIdentifier pd_A03

Name "A03_def"

Meta-relationship ...... DFMProcess.References.DFMProcessDefinition

Meta-attributes

CDIFIdentifier .........dfmp_A03.r.pd_A03

SourceEntity ..........dfmp_A03

DestinationEntity .........pd_A03

Meta-entity ......... FlowInputPort

Meta-attributes

CDIFIdentifier ......... fip_Af

IsFormal .......... TRUE

Meta-relationship ...... DFMProcessDefinition.Contains.FlowInputPort

Meta-attributes

CDIFIdentifier .........pd_A0.c.fip_Af

SourceEntity .......... pd_A0

DestinationEntity ....... fip_Af

Meta-relationship ..... FlowInputPort.References.FlowDefinition

Meta-attributes

CDIFIdentifier $\ldots \ldots$. . fip_Af.r.fd_A

SourceEntity ......... fip_Af

DestinationEntity ........fd_A

Meta-relationship ..... ReferencedElement.DefinesPath.ComponentObject

Meta-attributes

CDIFIdentifier ........ re_A.dp.fip_Af

SourceEntity ........... re_A

DestinationEntity ....... fip_Af

SequenceNumber ....... 2

$1 *$ Flow A */

Meta-entity ......... Flow

Meta-attributes

CDIFIdentifier ........f.A'

Name ............. "A" 
Meta-relationship ..... DFMProcessDefinition.Contains.Flow

Meta-attributes

CDIFIdentifier $\ldots \ldots \ldots$. pd_A0.c.f_A'

SourceEntity .......... pd_A0

DestinationEntity ........ f_A'

Meta-relationship ...... Flow.References.FlowDefinition

Meta-attributes

CDIFIdentifier ........f_A'.r.fd_A

SourceEntity ............ f_A'

DestinationEntity .......fd_A

Meta-relationship .... . FlowProducerConsumer.Consumes.FlowInputPort

Meta-attributes

CDIFIdentifier .........f_Af.c.fip_Af

SourceEntity ........... f_A'

DestinationEntity ....... fip_Af

Meta-relationship ..... FlowProducerConsumer.Produces.Flow

Meta-attributes

CDIFIdentifier ........ fip_Af.p.f_A'

SourceEntity ......... fip_Af

DestinationEntity ......... f_A'

Meta-entity ......... FlowInputPort

Meta-attributes

CDIFIdentifier ......... fip_A'a

IsFormal . . . . . . . . . FALSE

Meta-relationship ..... . DFMProcessDefinition.Contains.FlowInputPort

Meta-attributes

CDIFIdentifier .........pd_A0.c.cp_fip_A'a

SourceEntity ............ pd_A0

DestinationEntity ....... fip_A'a

Meta-relationship .... . FlowInputPort.References.FlowDefinition

Meta-attributes

CDIFIdentifier ........ fip_A'a.c.fd_A

SourceEntity .......... fip_A'a

DestinationEntity ........ fd_A 
Meta-relationship ..... FlowProducerConsumer.Consumes.Flow

Meta-attributes

CDIFIdentifier ....... fip_A'a.c.f_A'

SourceEntity ......... fip_A'a

DestinationEntity ........f_A'

Meta-relationship .... . . FlowProducerConsumer.Produces.FlowInputPort

Meta-attributes

CDIFIdentifier ......... f_A'.p.fip_A'a

SourceEntity .................

DestinationEntity $\ldots \ldots$. fip_A'a

Meta-entity ......... EquivalenceSet

Meta-attributes

CDIFIdentifier .........es_A'

Meta-relationship ..... . DFMProcessDefinition.Contains.EquivalenceSet

Meta-attributes

CDIFIdentifier $\ldots \ldots \ldots$ pd_A0.c.es_A'

SourceEntity ........... pd_A0

DestinationEntity ........es_A'

Meta-relationship ..... EquivalenceSet.HasMember.FlowInputPort

Meta-attributes

CDIFIdentifier $\ldots \ldots \ldots$ es_A'.hm.fip_A'a

SourceEntity .......... es_A'

DestinationEntity ....... fip_A'a

Meta-entity ......... ReferencedElement

Meta-attributes

CDIFIdentifier ......... re_A'

Meta-relationship ...... DFMProcessDefinition.Contains.ReferencedElement

Meta-attributes

CDIFIdentifier .........pd_A0.c.re_A'

SourceEntity ........... pd_A0

DestinationEntity $\ldots \ldots$.... re_A

Meta-relationship ...... EquivalenceSet.HasMember.ReferencedElement

Meta-attributes

CDIFIdentifier $\ldots \ldots \ldots$ es_A'.hm.re_A'

SourceEntity ........... es_A'

DestinationEntity ........ re_A' 
Meta-relationship ..... ReferencedElement.DefinesPath.ComponentObject

Meta-attributes

CDIFIdentifier .........re_A'.dp.dfmp_A01

SourceEntity .......... re_A'

DestinationEntity ........dfmp_A01

SequenceNumber ....... 1

Meta-entity ......... FlowInputPort

Meta-attributes

CDIFIdentifier ......... fip_A'f

IsFormal $\ldots \ldots \ldots \ldots$ TRUE

Meta-relationship ..... . DFMProcessDefinition.Contains.FlowInputPort

Meta-attributes

CDIFIdentifier ..........pd_A01.c.fip_A'f

SourceEntity .......... pd_A01

DestinationEntity $\ldots .$. . fip_A'f

Meta-relationship ..... . FlowInputPort.References.FlowDefinition

Meta-attributes

CDIFIdentifier ......... fip_A'f.r.fd_A

SourceEntity .......... fip_A'f

DestinationEntity ........ fd_A

Meta-relationship ..... ReferencedElement.DefinesPath.ComponentObject

Meta-attributes

CDIFIdentifier ........ re_A'.dp.fip_A'f

SourceEntity ........... re_A'

DestinationEntity ....... fip_A'f

SequenceNumber .......2

/* Flow A"*/

Meta-entity ......... Flow

Meta-attributes

CDIFIdentifier .........f.

Name ............. "A"

Meta-relationship ..... DFMProcessDefinition.Contains.Flow

Meta-attributes

CDIFIdentifier .........pd_A0.c.f_A"

SourceEntity $\ldots \ldots \ldots \ldots$ pd_A0

DestinationEntity ......... f_A" 
Meta-relationship ...... Flow.References.FlowDefinition

Meta-attributes

CDIFIdentifier .........f_A".r.fd_A

SourceEntity ...............

DestinationEntity ....... fd_A

Meta-relationship ..... FlowProducerConsumer.Consumes.FlowInputPort

Meta-attributes

CDIFIdentifier .................fip_Af

SourceEntity ..........f_"

DestinationEntity ........ fip_Af

Meta-relationship ..... FlowProducerConsumer.Produces.Flow

Meta-attributes

CDIFIdentifier ......... fip_Af.p.f_A"

SourceEntity ........... fip_Af

DestinationEntity ........ f_A"

Meta-entity ......... FlowInputPort

Meta-attributes

CDIFIdentifier ......... fip_A"a

IsFormal ............ FALSE

Meta-relationship ..... DFMProcessDefinition.Contains.FlowInputPort

Meta-attributes

CDIFIdentifier .........pd_A0.c.cp_fip_A"a

SourceEntity ............ pd_A0

DestinationEntity $\ldots \ldots$. fip_A"a

Meta-relationship ..... . FlowInputPort.References.FlowDefinition

Meta-attributes

CDIFIdentifier ........ fip_A"a.c.fd_A

SourceEntity ......... fip_A"a

DestinationEntity ........fd_A

Meta-relationship ..... FlowProducerConsumer.Consumes.Flow

Meta-attributes

CDIFIdentifier ........ fip_A"a.c.f_A"

SourceEntity .......... fip_A"a

DestinationEntity ........f_A" 
Meta-relationship ..... . FlowProducerConsumer.Produces.FlowInputPort Meta-attributes

CDIFIdentifier $\ldots \ldots \ldots$ f_A".p.fip_A"a

SourceEntity .......... f_A"

DestinationEntity ........ fip_A"a

Meta-entity ......... EquivalenceSet

Meta-attributes

CDIFIdentifier .........es_A"

Meta-relationship ..... . DFMProcessDefinition.Contains.EquivalenceSet

Meta-attributes

CDIFIdentifier $\ldots \ldots \ldots$ pd_A0.c.es_A"

SourceEntity ........... pd_A0

DestinationEntity .......es_A"

Meta-relationship ..... . EquivalenceSet.HasMember.FlowInputPort

Meta-attributes

CDIFIdentifier $\ldots \ldots \ldots$ es_A".hm.fip_A"a

SourceEntity ........... es_A"

DestinationEntity ....... fip_A"a

Meta-entity ......... ReferencedElement

Meta-attributes

CDIFIdentifier ......... re_A"

Meta-relationship ..... . DFMProcessDefinition.Contains.ReferencedElement

Meta-attributes

CDIFIdentifier ........pd_A0.c.re_A"

SourceEntity ........... pd_A0

DestinationEntity ....... re_A"

Meta-relationship ..... EquivalenceSet.HasMember.ReferencedElement

Meta-attributes

CDIFIdentifier .........es_A".hm.re_A"

SourceEntity .......... es_A"

DestinationEntity ....... re_A"

Meta-relationship ..... ReferencedElement.DefinesPath.ComponentObject

Meta-attributes

CDIFIdentifier ........re_A".dp.dfmp_A02

SourceEntity .......... re_A"

DestinationEntity .......dfmp_A02

SequenceNumber ........ 1 
Meta-entity ......... FlowInputPort

Meta-attributes

CDIFIdentifier ......... fip_A"f

IsFormal $\ldots \ldots \ldots \ldots$ TRUE

Meta-relationship ... . . DFMProcessDefinition.Contains.FlowInputPort

Meta-attributes

CDIFIdentifier .........pd_A02.c.fip_A"f

SourceEntity ............pd_A02

DestinationEntity ........ fip_A"f

Meta-relationship ..... . FlowInputPort.References.FlowDefinition

Meta-attributes

CDIFIdentifier ........ fip_A"f.r.fd_A

SourceEntity ......... fip_A"f

DestinationEntity ....... fd_A

Meta-relationship ..... ReferencedElement.DefinesPath.ComponentObject

Meta-attributes

CDIFIdentifier $\ldots \ldots \ldots$ re_A".dp.fip_A"f

SourceEntity .......... re_A"

DestinationEntity ........ fip_A"f

SequenceNumber .......2 


\subsection{2 "A Copy" Flow Decomposition}

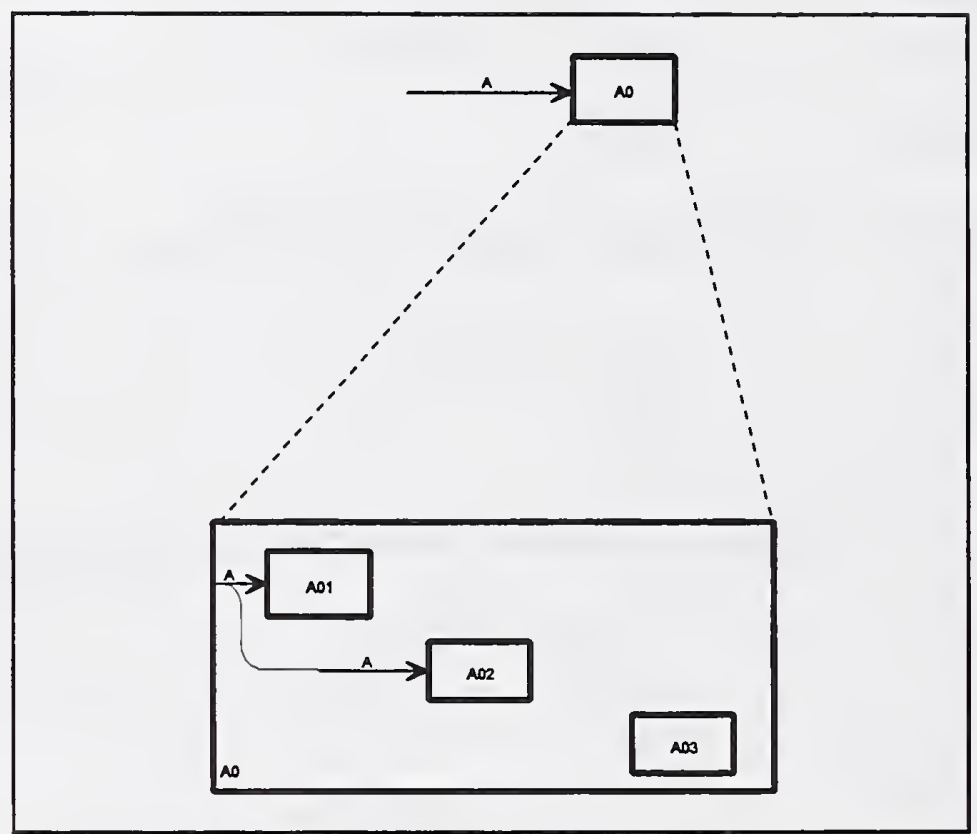

Figure 16. "A Copy" Decomposition

The only difference between a "Blind Copy" and "A Copy" decomposition is that in the "Copy" decomposition we can "see" the flow split. So, in this case, FlowInputPort Af produces Flow A', which then produces flows A" and A"'.

The following is the CDIF mapping of the above decomposition. A graphical representation of the mapping can be found in the Appendix A.

Meta-entity ......... DFMProcess

Meta-attributes

CDIFIdentifier ........ dfmp_A0

Name ............ "A0"

Meta-entity ........ DFMProcessDefinition

Meta-attributes

CDIFIdentifier .......... pd_AO

Name ............ A0_def" 
Meta-relationship ..... . DFMProcess.References.DFMProcessDefinition

Meta-attributes

CDIFIdentifier $\ldots$.....dfmp_A0.r.pd_A0

SourceEntity .......... dfmp_A0

DestinationEntity $\ldots \ldots \ldots$ pd_A0

Meta-entity ......... Flow

Meta-attributes

CDIFIdentifier ............ $\mathrm{f}_{-} \mathrm{A}$

Name ............" A" $^{n}$

Meta-entity ......... FlowInputPort

Meta-attributes

CDIFIdentifier ......... fip_Aa

IsFormal $\ldots \ldots \ldots \ldots \ldots$ FALSE

Meta-relationship ..... FlowInputPort.Consumes.Flow

Meta-attributes

CDIFIdentifier ........ fip_Aa.c.f_A

SourceEntity .......... fip_Aa

DestinationEntity ......... f_A

Meta-relationship ..... Flow.Produces.FlowInputPort

Meta-attributes

CDIFIdentifier ................fip_Aa

SourceEntity ............A

DestinationEntity ........ fip_Aa

Meta-entity ......... EquivalenceSet

Meta-attributes

CDIFIdentifier ..........es_A

Meta-relationship ..... EquivalenceSet.HasMember.FlowInputPort

Meta-attributes

CDIFIdentifier .........es_A.hm.fip_Aa

SourceEntity .......... es_A

DestinationEntity ....... fip_Aa

Meta-entity ......... ReferencedElement

Meta-attributes

CDIFIdentifier .........re_A 
Meta-relationship ..... EquivalenceSet.HasMember.ReferencedElement

Meta-attributes

CDIFIdentifier $\ldots \ldots \ldots$ es_A.hm.re_A

SourceEntity ............ es_A

DestinationEntity..... re_A

Meta-relationship ..... ReferencedElement.DefinesPath.ComponentObject

Meta-attributes

CDIFIdentifier $\ldots \ldots$.... re_A.dp.dfmp_A0

SourceEntity ........... re_A

DestinationEntity .......dfmp_A0

SequenceNumber ........

Meta-entity ......... FlowDefinition

Meta-attributes

CDIFIdentifier ........... fd_A

Name .............. "A_def"

Meta-relationship ..... Flow.References.FlowDefinition

Meta-attributes

CDIFIdentifier ........f_A.r.fd_A

SourceEntity ........... f_A

DestinationEntity ........fd_A

Meta-relationship ..... . FlowInputPort.References.FlowDefinition

Meta-attributes

CDIFIdentifier ......... fip_Aa.r.fd_A

SourceEntity .......... fip_Aa

DestinationEntity ........fd_A

/* Process Decomposition: the next level of abstraction. */

Meta-entity ......... DFMProcess

Meta-attributes

CDIFIdentifier ........dfmp_A01

Name ............. "A01"

Meta-entity ......... DFMProcessDefinition.Contains.DFMProcess

Meta-attributes

CDIFIdentifier .........pd_A0.c.dfmp_A01

SourceEntity .......... pd_A0

DestinationEntity ........ dfmp_A01 
Meta-entity ......... DFMProcessDefinition

Meta-attributes

CDIFIdentifier ...........pd_A01

Name "A01_def"

Meta-relationship ...... DFMProcess.References.DFMProcessDefinition

Meta-attributes

CDIFIdentifier .........dfmp_A01.r.pd_A01

SourceEntity .........dfmp_A01

DestinationEntity .........pd_A01

Meta-entity ......... DFMProcess

Meta-attributes

CDIFIdentifier ........dfmp_A02

Name ............. "A02"

Meta-entity ........ DFMProcessDefinition.Contains.DFMProcess

Meta-attributes

CDIFIdentifier ........pd_A0.c.dfmp_A02

SourceEntity ........... pd_A0

DestinationEntity .......dfmp_A02

Meta-entity ........ DFMProcessDefinition

Meta-attributes

CDIFIdentifier ..........pd_A02

Name ............ "A02_def"

Meta-relationship ..... DFMProcess.References.DFMProcessDefinition

Meta-attributes

CDIFIdentifier .........dfmp_A02.r.pd_A02

SourceEntity .......... dfmp_A02

DestinationEntity $\ldots \ldots$....pd_A02

Meta-entity ......... DFMProcess

Meta-attributes

CDIFIdentifier .........dfmp_A03

Name ............"A03"

Meta-entity ........ DFMProcessDefinition.Contains.DFMProcess

Meta-attributes

CDIFIdentifier .........pd_A0.c.dfmp_A03

SourceEntity $\ldots \ldots \ldots \ldots$. pd_A0

DestinationEntity .......dfmp_A03 
Meta-entity ........ DFMProcessDefinition

Meta-attributes

CDIFIdentifier ...........pd_A03

Name "A03_def"

Meta-relationship ...... DFMProcess.References.DFMProcessDefinition

Meta-attributes

CDIFIdentifier $\ldots$......dfmp_A03.r.pd_A03

SourceEntity ..........dfmp_A03

DestinationEntity .........pd_A03

Meta-entity ......... FlowInputPort

Meta-attributes

CDIFIdentifier ......... fip_Af

IsFormal ........... TRUE

Meta-relationship ..... . DFMProcessDefinition.Contains.FlowInputPort

Meta-attributes

CDIFIdentifier $\ldots$........pd_A0.c.fip_Af

SourceEntity .......... pd_A0

DestinationEntity ........ fip_Af

Meta-relationship ..... FlowInputPort.References.FlowDefinition

Meta-attributes

CDIFIdentifier ........ fip_Af.r.fd_A

SourceEntity .......... fip_Af

DestinationEntity ........fd_A

Meta-relationship ...... ReferencedElement.DefinesPath.ComponentObject

Meta-attributes

CDIFIdentifier .........re_A.dp.fip_Af

SourceEntity .......... re_A

DestinationEntity ...... fip_Af

SequenceNumber ....... 2

/* Flow A'*/

Meta-entity ......... Flow

Meta-attributes

CDIFIdentifier ........f.

Name ............ "A" 
Meta-relationship ..... DFMProcessDefinition.Contains.Flow

Meta-attributes

CDIFIdentifier $\ldots \ldots \ldots$ pd_A0.c.f_A'

SourceEntity .......... pd_A0

DestinationEntity ...............

Meta-relationship ..... Flow.References.FlowDefinition

Meta-attributes

CDIFIdentifier .........f_A'.r.fd_A

SourceEntity ...............

DestinationEntity .......fd_A

Meta-relationship .... . FlowProducerConsumer.Consumes.FlowInputPort

Meta-attributes

CDIFIdentifier ........f_. $A^{\prime}$.c.fip_Af

SourceEntity .......... f_A $A^{\prime}$

DestinationEntity .......ffip_Af

Meta-relationship .... . FlowProducerConsumer.Produces.Flow

Meta-attributes

CDIFIdentifier ........ fip_Af.p.f_A'

SourceEntity ...........fip_Af

DestinationEntity ....... f_A

/ Flow A"*/

Meta-entity .......... Flow

Meta-attributes

CDIFIdentifier ........f. f_A"

Name .............." $A^{\prime \prime}$

Meta-relationship ..... DFMProcessDefinition.Contains.Flow

Meta-attributes

CDIFIdentifier ........pd_A0.c.f_A"

SourceEntity ..........pd_A0

DestinationEntity ........f_A"

Meta-relationship ..... Flow.References.FlowDefinition

Meta-attributes

CDIFIdentifier .........f_A".r.fd_A

SourceEntity ..........f_A"

DestinationEntity .......fd_A 
Meta-relationship ... . . FlowProducerConsumer.Consumes.Flow

Meta-attributes

CDIFIdentifier .........f_A".c.f__A'

SourceEntity ................

DestinationEntity ........ f_A

Meta-relationship .... . FlowProducerConsumer.Produces.Flow

Meta-attributes

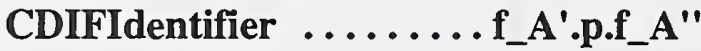

SourceEntity ................. $\mathbf{A}^{\prime}$

DestinationEntity ........ f_A"

Meta-entity ........ FlowInputPort

Meta-attributes

CDIFIdentifier ......... fip_A"a

IsFormal ............. FALSE

Meta-relationship ..... . DFMProcessDefinition.Contains.FlowInputPort

Meta-attributes

CDIFIdentifier .........pd_A0.c.cp_fip_A"a

SourceEntity ........... pd_A0

DestinationEntity ....... fip_A"a

Meta-relationship ...... FlowInputPort.References.FlowDefinition

Meta-attributes

CDIFIdentifier ........ fip_A"a.c.fd_A

SourceEntity ......... fip_A"a

DestinationEntity .......fd_A

Meta-relationship ..... FlowProducerConsumer.Consumes.Flow

Meta-attributes

CDIFIdentifier ........ fip_A"a.c.f_A"

SourceEntity ......... fip_A"a

DestinationEntity ...........A"

Meta-relationship ..... . FlowProducerConsumer.Produces.FlowInputPort

Meta-attributes

CDIFIdentifier $\ldots \ldots \ldots$ f_A".p.fip_A"a

SourceEntity ..............."

DestinationEntity ........fip_A"a

Meta-entity ......... EquivalenceSet

Meta-attributes

CDIFIdentifier .........es_A" 
Meta-relationship ..... DFMProcessDefinition.Contains.EquivalenceSet

Meta-attributes

CDIFIdentifier $\ldots \ldots \ldots$ pd_A0.c.es_A"

SourceEntity ........... pd_A0

DestinationEntity .......e es_A"

Meta-relationship . . . . E EquivalenceSet.HasMember.FlowInputPort

Meta-attributes

CDIFIdentifier ........es_A".hm.fip_A"a

SourceEntity ..........es_A"

DestinationEntity $\ldots \ldots$. fip_A"a

Meta-entity ......... ReferencedElement

Meta-attributes

CDIFIdentifier .......... re_A"

Meta-relationship ..... . DFMProcessDefinition.Contains.ReferencedElement

Meta-attributes

CDIFIdentifier .........pd_A0.c.re_A"

SourceEntity ........... pd_A0

DestinationEntity ....... re_A"

Meta-relationship ..... EquivalenceSet.HasMember.ReferencedElement

Meta-attributes

CDIFIdentifier $\ldots \ldots \ldots$ es_A".hm.re_A"

SourceEntity ..........es_A"

DestinationEntity ....... re_A"

Meta-relationship ..... ReferencedElement.DefinesPath.ComponentObject

Meta-attributes

CDIFIdentifier ........ re_A".dp.dfmp_A01

SourceEntity ..........re_A"

DestinationEntity .......dfmp_A01

SequenceNumber ....... 1

Meta-entity ......... FlowInputPort

Meta-attributes

CDIFIdentifier .........fip_A"f

IsFormal $\ldots \ldots \ldots \ldots$ TRUE 
Meta-relationship ...... DFMProcessDefinition.Contains.FlowInputPort

Meta-attributes

CDIFIdentifier $\ldots \ldots \ldots$. pd_A01.c.fip_A"f

SourceEntity ............ pd_A01

DestinationEntity ....... fip_A"f

Meta-relationship ...... FlowInputPort.References.FlowDefinition

Meta-attributes

CDIFIdentifier ........ fip_A"f.r.fd_A

SourceEntity ........... fip_A"f

DestinationEntity .......fd_A

Meta-relationship ..... ReferencedElement.DefinesPath.ComponentObject

Meta-attributes

CDIFIdentifier .........re_A".dp.fip_A"f

SourceEntity .......... re_A"

DestinationEntity ........ fip_A"f

SequenceNumber .......2

/* Flow A"**/

Meta-entity ......... Flow

Meta-attributes

CDIFIdentifier ........f. $\mathrm{f} \mathrm{A}^{\prime \prime}$

Name .............." "A"

Meta-relationship ...... DFMProcessDefinition.Contains.Flow

Meta-attributes

CDIFIdentifier $\ldots \ldots \ldots$. pd_A0.c.f_A'"

SourceEntity ..........pd_A0

DestinationEntity $\ldots \ldots \ldots$ f_A"'

Meta-relationship ..... . Flow.References.FlowDefinition

Meta-attributes

CDIFIdentifier .........f_A'".r.fd_A

SourceEntity ..............'

DestinationEntity ........ fd_A

Meta-relationship ..... FlowProducerConsumer.Consumes.Flow

Meta-attributes

CDIFIdentifier ..............."'.c.f_A'

SourceEntity ...............'

DestinationEntity ............. 
Meta-relationship ..... FlowProducerConsumer.Produces.Flow

Meta-attributes

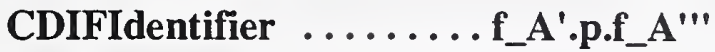

SourceEntity ................'

DestinationEntity ........f_."'

Meta-entity ......... FlowInputPort

Meta-attributes

CDIFIdentifier ......... fip_A"'a

IsFormal ............ FALSE

Meta-relationship ..... . DFMProcessDefinition.Contains.FlowInputPort

Meta-attributes

CDIFIdentifier $\ldots \ldots \ldots$. pd_A0.c.cp_fip_A"'a

SourceEntity .......... pd_A0

DestinationEntity ........ fip_A"'a

Meta-relationship ..... FlowInputPort.References.FlowDefinition

Meta-attributes

CDIFIdentifier $\ldots \ldots \ldots$. fip_A"'a.c.fd_A

SourceEntity .......... fip_A"'a

DestinationEntity $\ldots . .$. fd_A

Meta-relationship ..... FlowProducerConsumer.Consumes.Flow

Meta-attributes

CDIFIdentifier $\ldots \ldots \ldots$ fip_A"'a.c.f_A"'

SourceEntity .......... fip_A"'a

DestinationEntity ........ f_A"'

Meta-relationship ..... FlowProducerConsumer.Produces.FlowInputPort

Meta-attributes

CDIFIdentifier .........f_A"'.p.fip_A"'a

SourceEntity ......... f_A"'

DestinationEntity ........ fip_A"'a

Meta-entity ......... EquivalenceSet

Meta-attributes

CDIFIdentifier $\ldots . .$. . es_A"'

Meta-relationship ...... DFMProcessDefinition.Contains.EquivalenceSet

Meta-attributes

CDIFIdentifier $\ldots \ldots \ldots$. pd_A0.c.es_A"'

SourceEntity .......... pd_A0

DestinationEntity ........es_A"' 
Meta-relationship ..... EquivalenceSet.HasMember.FlowInputPort

Meta-attributes

CDIFIdentifier ….....es_A"'.hm.fip_A"'a

SourceEntity .......... es_A"'

DestinationEntity ........ fip_A"'a

Meta-entity ......... ReferencedElement

Meta-attributes

CDIFIdentifier .........re_A"'

Meta-relationship ..... DFMProcessDefinition.Contains.ReferencedElement

Meta-attributes

CDIFIdentifier $\ldots \ldots \ldots$. pd_A0.c.re_A"'

SourceEntity .......... pd_A0

DestinationEntity ........re_e"'

Meta-relationship ...... EquivalenceSet.HasMember.ReferencedElement

Meta-attributes

CDIFIdentifier $\ldots \ldots$. . es_A"'.hm.re_A"'

SourceEntity ..........e. es_A"'

DestinationEntity ........re_A"'

Meta-relationship ..... ReferencedElement.DefinesPath.ComponentObject

Meta-attributes

CDIFIdentifier $\ldots \ldots \ldots$. re_A"'.dp.dfmp_A02

SourceEntity .......... re_A"'

DestinationEntity .......dfmp_A02

SequenceNumber ....... 1

Meta-entity ......... FlowInputPort

Meta-attributes

CDIFIdentifier .......... fip_A"'f

IsFormal $\ldots \ldots \ldots \ldots$ TRUE

Meta-relationship ..... DFMProcessDefinition.Contains.FlowInputPort

Meta-attributes

CDIFIdentifier $\ldots \ldots \ldots$. pd_A02.c.fip_A"'f

SourceEntity ............pd_A02

DestinationEntity ....... fip_A"'f 
Meta-relationship ..... FlowInputPort.References.FlowDefinition

Meta-attributes

CDIFIdentifier ........ fip_A'"f.r.fd_A

SourceEntity ......... fip_A"'f

DestinationEntity .......fd_A

Meta-relationship ..... ReferencedElement.DefinesPath.ComponentObject

Meta-attributes

CDIFIdentifier $\ldots \ldots \ldots$. re_A'".dp.fip_A"'f

SourceEntity .......... re_A"'

DestinationEntity ....... fip_A"'f

SequenceNumber .......2

\subsection{3 "Blind Portion" Flow Decomposition}

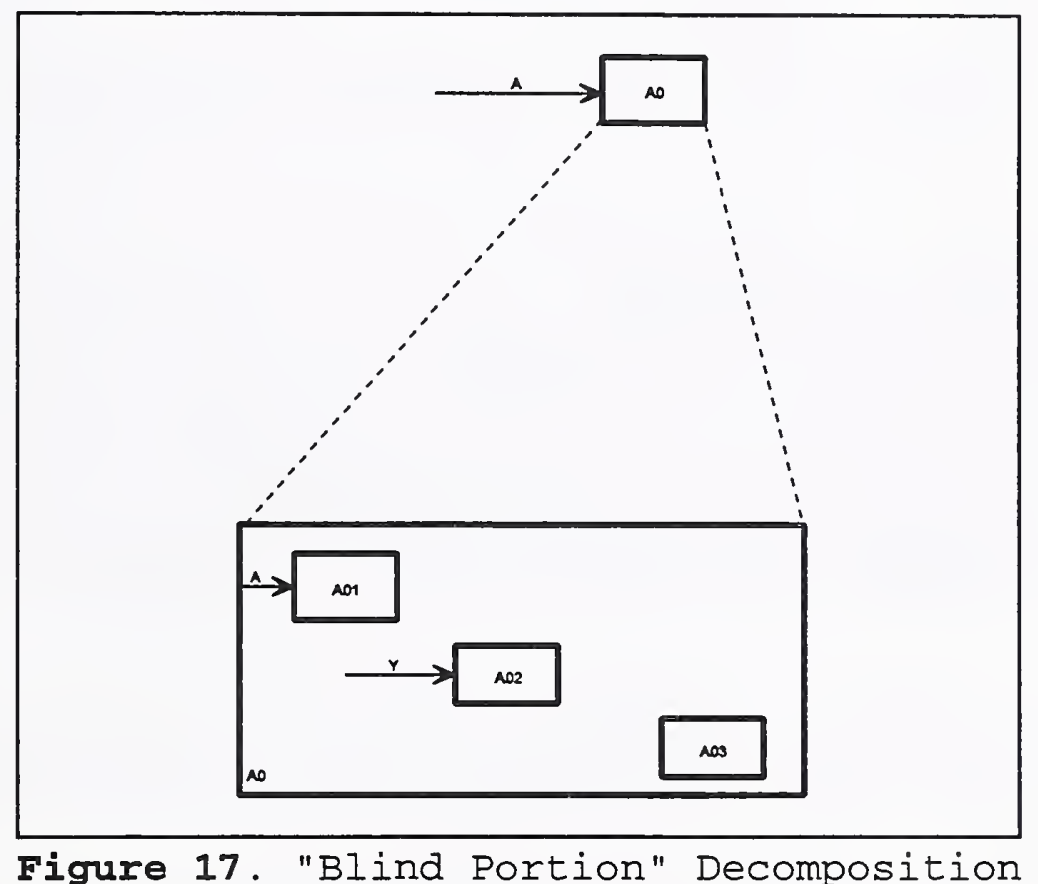

Like in the "Blind Copy" decomposition, we do not "see" the split of the flow, but we assume it occurred. In this case, Flow A contains Flow Y in its structure. We need to have a separate FlowDefinition for Y. Also, we have to define the structure of the flow A by creating an extra instance $A_{-} Y$ of flow $Y$ and showing that $A_{-} Y$ is in the structure of 
A through the means of meta-relationship FlowDefinition.Contains.Flow (see Appendix A).

We also need to link that A_Y with the actual flow $\mathrm{Y}$ to define $\mathrm{Y}$. This is done through the EquivalenceSet and ReferencedElement.

A graphical representation of the mapping can be found in the Appendix A.

\subsection{4 "A Portion" Flow Decomposition}

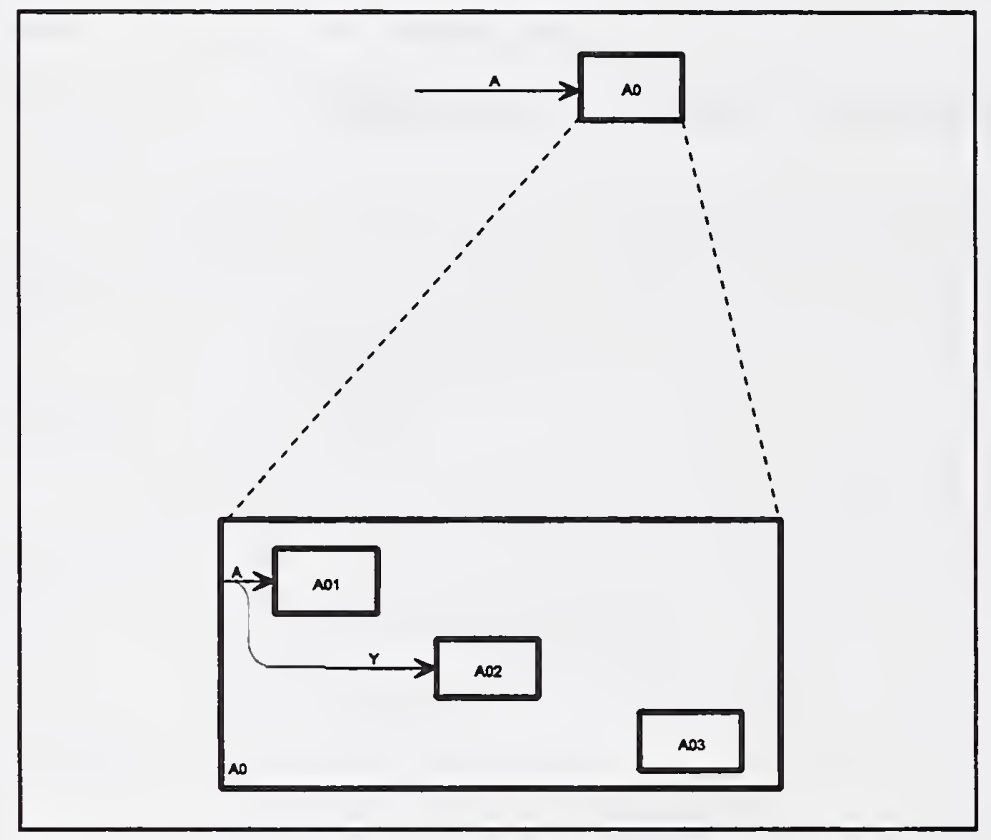

Figure 18. "A Portion" Decomposition

The only difference between a "Blind Portion" and "A Portion" decomposition is that in the "Portion" decomposition we can "see" the flow split. So, in this case, FlowInputPort Af produces Flow A', which then produces flows A" and Y.

A graphical representation of the mapping can be found in the Appendix A. 


\subsection{5 "Blind Sum" Flow Decomposition}

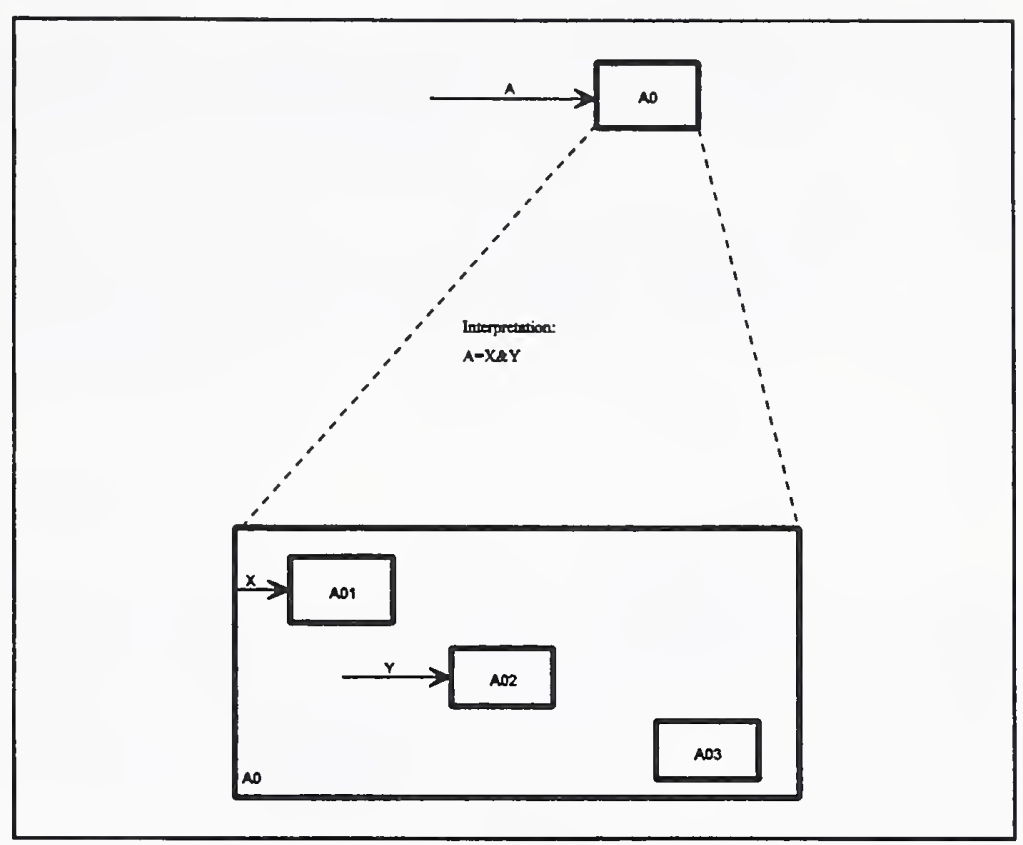

Figure 19. "Blind Sum" Decomposition

Like in the "Blind Copy" and "Blind Portion" decompositions, we do not "see" the split of the flow, but we assume it occured. In this case, Flow A contains Flow X and Flow Y in its structure. We need to have a separate FlowDefinition for A, X, and Y. Also, we have to define the structure of the flow A by creating an extra instance A_X and A_Y of flow $\mathrm{X}$ and $\mathrm{Y}$, respectively, and showing that A_X and A_Y are in the structure of A through the means of meta-relationship FlowDefinition. Contains. Flow (see Appendix A).

We also need to link that A_X and A_Y with the actual flows X and $Y$ to define these flows. This is done through the EquivalenceSet and ReferencedElement for $\mathrm{X}$ and $\mathrm{Y}$.

A graphical representation of the mapping can be found in the Appendix A. 


\subsection{6 "The Sum" Flow Decomposition}

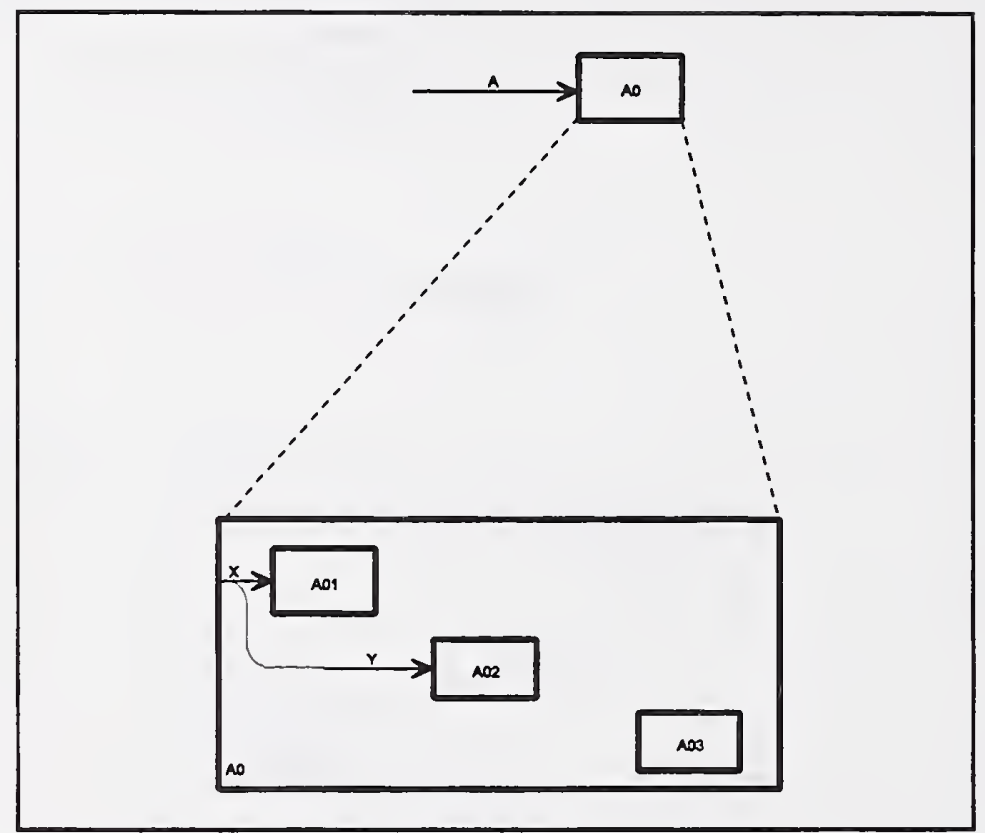

Figure 20. "The Sum" Decomposition

The only difference between a "Blind Sum" and "The Sum" decomposition is that in the "Sum" decomposition we can "see" the flow split. So, in this case, FlowInputPort Af produces Flow A', which then produces flows X and Y.

A graphical representation of the mapping can be found in the Appendix A. 


\subsection{SYNTACTICAL MAPPING}

\subsection{CDIF vs. IDL}

The following pages are compliant with the CDIF Presentation Location and Connectivity (PlaC) Subject Area, CDIF-JE-N15-DRAFT and the IDL language, Version 1.2.8.

In the CDIF PlaC, any subtype of the meta-entity GraphicalElement can be associated with its semantical representation through the meta-relationship GraphicalElement.Represents.RootObject. This would be the way, for example, to link a box to its process definition.

\subsubsection{Coordinate Frame}

The CDIF Transfer Coordinate Frame is a three dimensional system with the origin at $(0,0,0)$, assumed to be at the top left front corner, with $X, Y$, and $Z$ coordinate limits of minimum to the maximum of a CDIF integer. The positive $\mathrm{X}$-axis extends to the right, the positive $\mathrm{Y}$-axis extends downwards, and the positive $\mathrm{Z}$-axis extends to the back.

At this time most existing CASE tools are only two dimensional; however, to permit upward compatibility, a three demensional coordinate system was introduced. The $\mathrm{Z}$ coordinate shall have a value of 0 in all transfers that use two dimensional system ${ }^{4}$.

IDL uses two dimensional coordinate system. The origin of the IDL coordinate system is the upper left corner of the diagram message area. The point $(0.9999,0.9999)$ is the lower right corner of the message area.

\subsubsection{Kit}

Each separate page of IDEF0 KIT would be a Diagram. These pages can be "stapled" into a kit using Derivation meta-entity from the CDIF Common Subject Area.

\subsubsection{Diagram}

The CDIF PlaC Subject Area does not differentiate between different types of diagrams (IDL: d-type ::= 'GRAPHIC' I 'TEXT' I 'GLOSSARY' I 'FEO'). Also, it does not have

4 Throughout this document examples will be provided in two dimensions only. 
separate meta-attributes for IDL diag-head data which is AUTHOR, PROJECT, CREATION DATE, etc. However, the PlaC Subject Area has the meta-entity Annotation, which is a subtype of the GraphicalElement. An Annotation has the metaattributes TextBlock, BitMap, and ExtentX and Extent $Y$. The meta-attribute TextBlock may contain text (AUTHOR, PROJECT, CREATION DATE, etc), while BitMap may contain a bitmap for the grid of the text. The meta-attributes Extent $X$ and Extent $Y$ represent the location of the TextBlock or the BitMap. Either TextBlock, or BitMap may be given, but no combination of them is allowed.

Similar can be done about IDL status ('WORKING' I 'DRAFT' I etc.) and diag-body (IDL, pg. 8).

\subsubsection{Box}

The IDEF0 Box (Function) is represented through the CDIF meta-entity Node - the subtype of PositionedElement. A Node does not describe any attributes of the shape of the object. Instead, it captures the location and representation of the object.

All the text in a box can be put in the description of the ProcessDefinition or the DFMProcess, which then can be linked with the Node via the means of the metarelationship GraphicalElement.Represents.RootObject.

\subsubsection{Arrow}

A line on an IDEF0 diagram is represented using meta-entity $E d g e$, which provides the connectivity information, and EdgeElement, which represents one line segment with one source and one destination point. Through the means of the meta-relationship Edge.ConsistsOf.EdgeElement, an Edge can be built up from many EdgeElements. An EdgeElement can have only one single start and end point, and it has a local metaattribute ArrowOrientation ${ }^{5}$, which can be attached to the line segment.

5 There is an error in the PlaC Subject Area: the metaattribute Arroworientation is not listed in the attribute list of the meta-entity EdgeElement. 


\section{APPENDIX A: Figures}

Figure 1: Mapping IDEF Models Using CDIF $\quad \underline{86}$

Figure 2: Crossing an Abstraction Level $\quad \underline{87}$

Figure 3: IDEF0 A-0 Diagram $\quad \underline{\mathbf{8 8}}$

Figure 3-2: CDIF Mapping of A-O Diagram $\underline{\mathbf{8 9}}$

Figure 4: "A Copy" Fork and its CDIF Mapping $\quad \mathbf{9 0}$

Figure 5: "A Portion" Fork 21

Figure 5-2: CDIF Mapping of "A Portion" Fork 2

Figure 6: "The Sum" Fork 2

Figure 6-2: CDIF Mapping of "The Sum" Fork 24

Figure 7: "A Copy" Join and its CDIF Mapping 2

Figure 8: "A Portion" Join $\quad \mathbf{9 6}$

Figure 8-2: CDIF Mapping of "A Portion" Join 29

Figure 9: "The Sum" Join

Figure 9-2: CDIF Mapping of "The Sum" Join 29

Figure 10: "Another Data Model" Call $\underline{\mathbf{1 0 0}}$

Figure 11: "A Document" Call

Figure 12: SBT Call $\quad \underline{\mathbf{1 0 2}}$

Figure 13: A Drop in the Level of Abstraction Call $\underline{\mathbf{1 0 3}}$

Figure 14: Types of Flow Decomposition $\underline{\mathbf{1 0 4}}$

Figure 15: "Blind Copy" Decomposition $\underline{\mathbf{1 0 5}}$

Figure 15-2: CDIF Mapping of the "Blind Copy" Decomposition $\underline{\mathbf{1 0 6}}$

Figure 16: "A Copy" Decomposition $\underline{107}$

Figure 16-2: CDIF Mapping of the "Copy" Decomposition $\underline{108}$

Figure 17: "Blind Portion" Decomposition $\quad \underline{109}$

Figure 17-2: CDIF Mapping of the "Blind Portion" Decomposition

Figure 18: "A Portion" Decomposition $\underline{111}$

Figure 18-2: CDIF Mapping of the"Portion" Decomposition $\underline{112}$

Figure 19: "Blind Sum" Decomposition $\quad \underline{113}$

Figure 19-2: CDIF Mapping of the "Blind Sum" Decomposition

Figure 20: "The Sum" Decomposition $\underline{\mathbf{1 1 5}}$

Figure 20-2: CDIF Mapping of the "Sum" Decomposition $\quad \underline{116}$

Figure 21: CDIF Data Flow Model Subject Area $\quad \underline{117}$ 


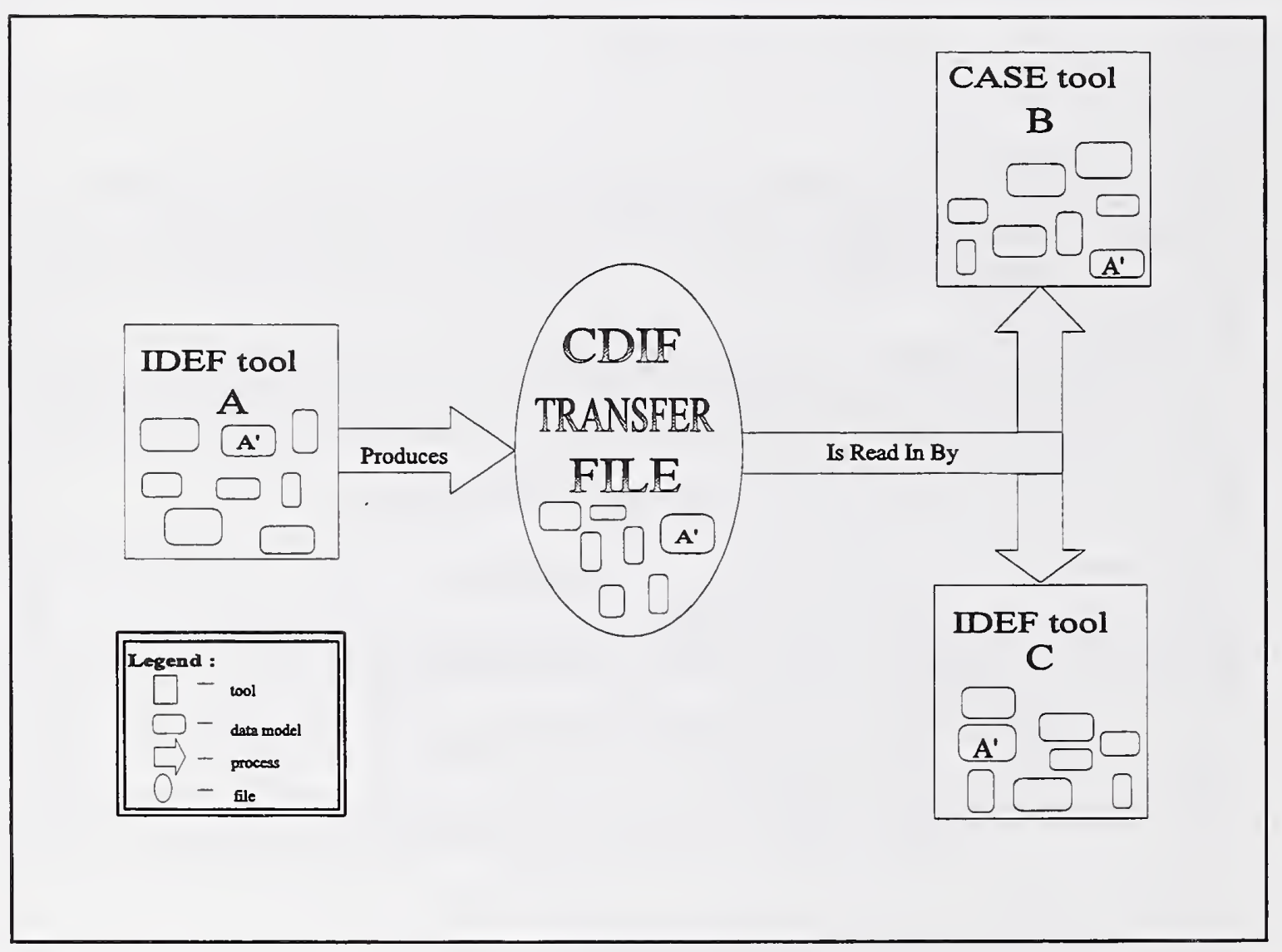

Figure 1: Mapping IDEF Models Using CDIF 


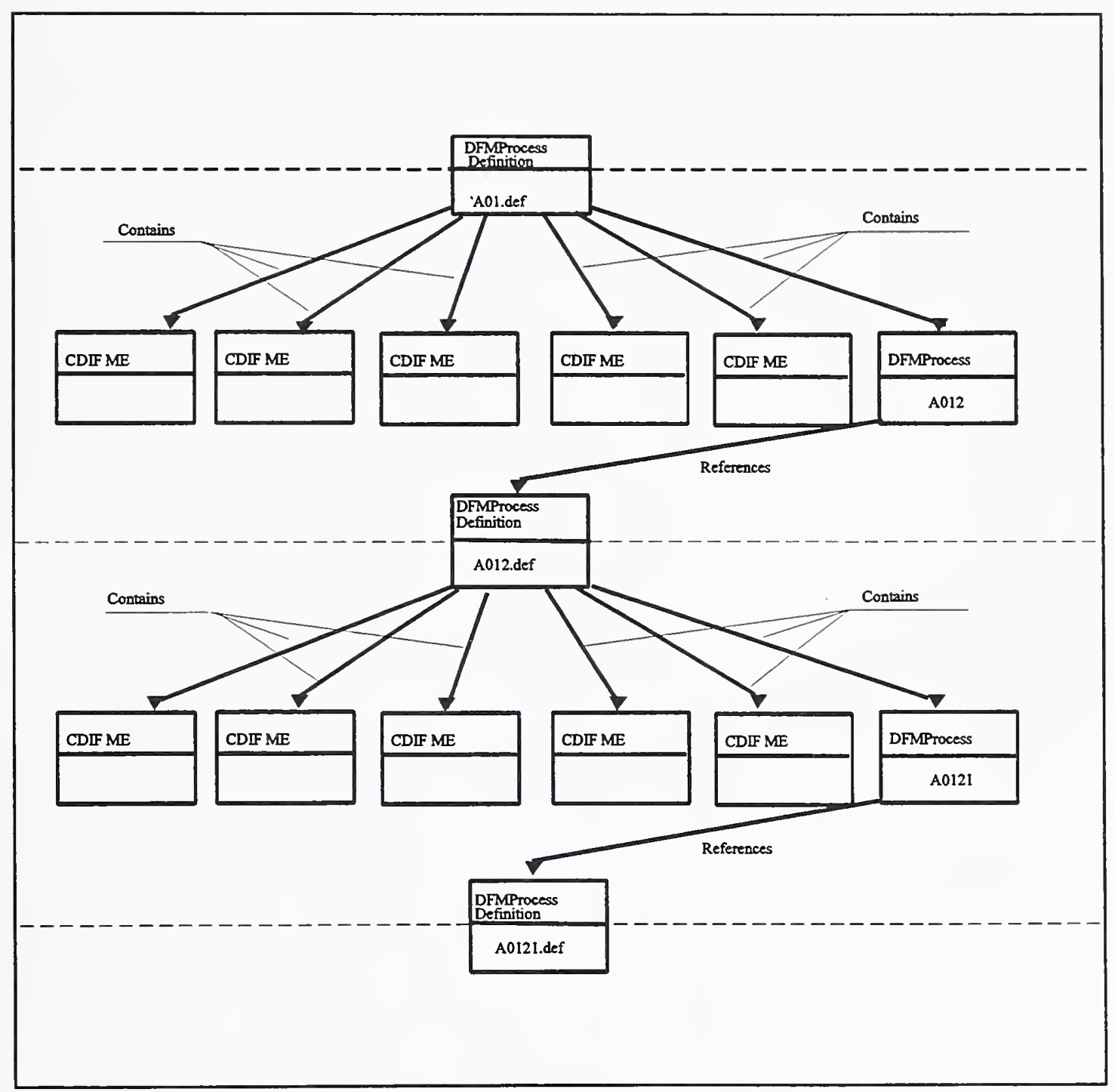

Figure 2: Crossing an Abstraction Level 


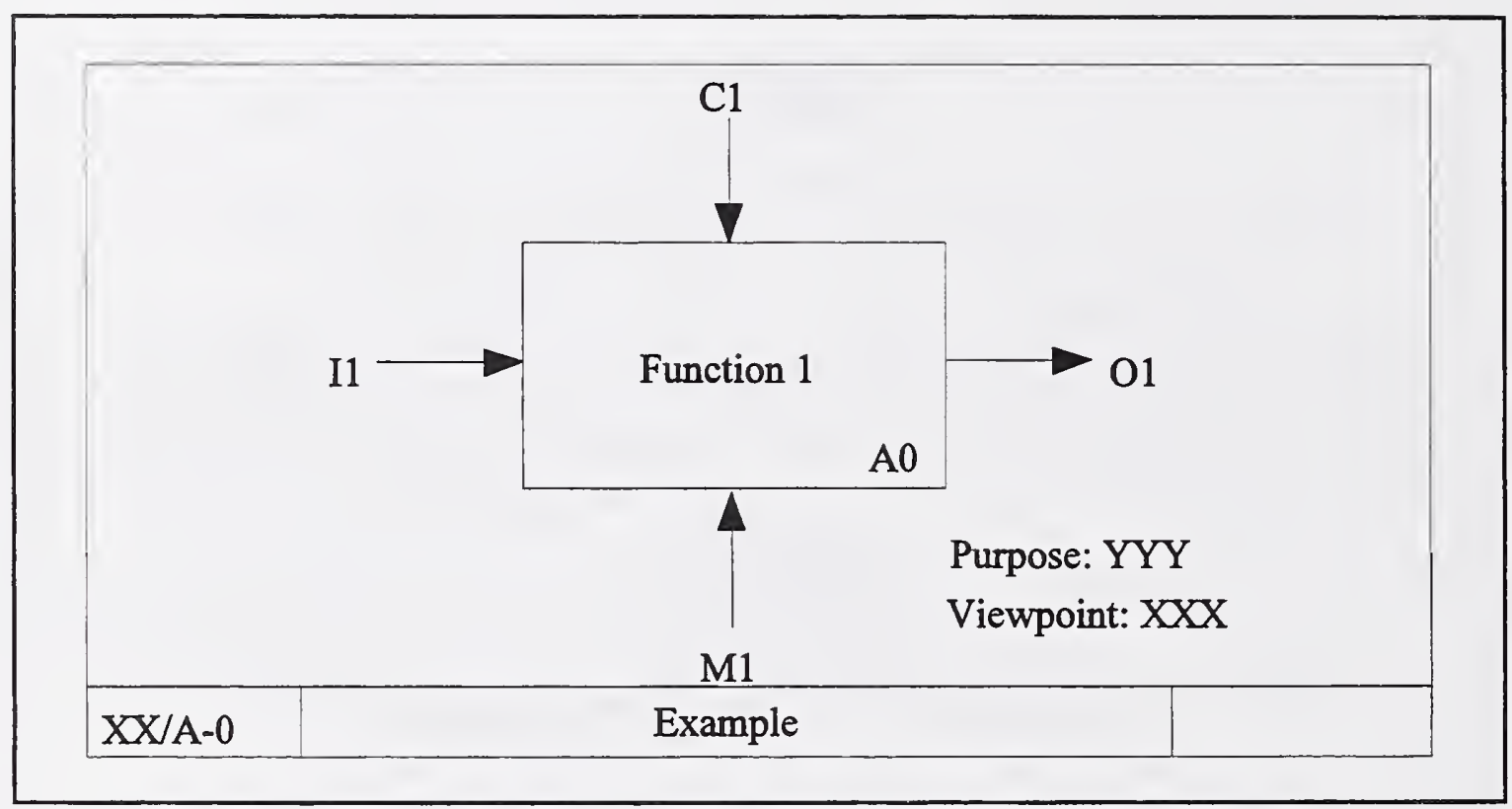

Figure 3: IDEF0 A-0 Diagram 


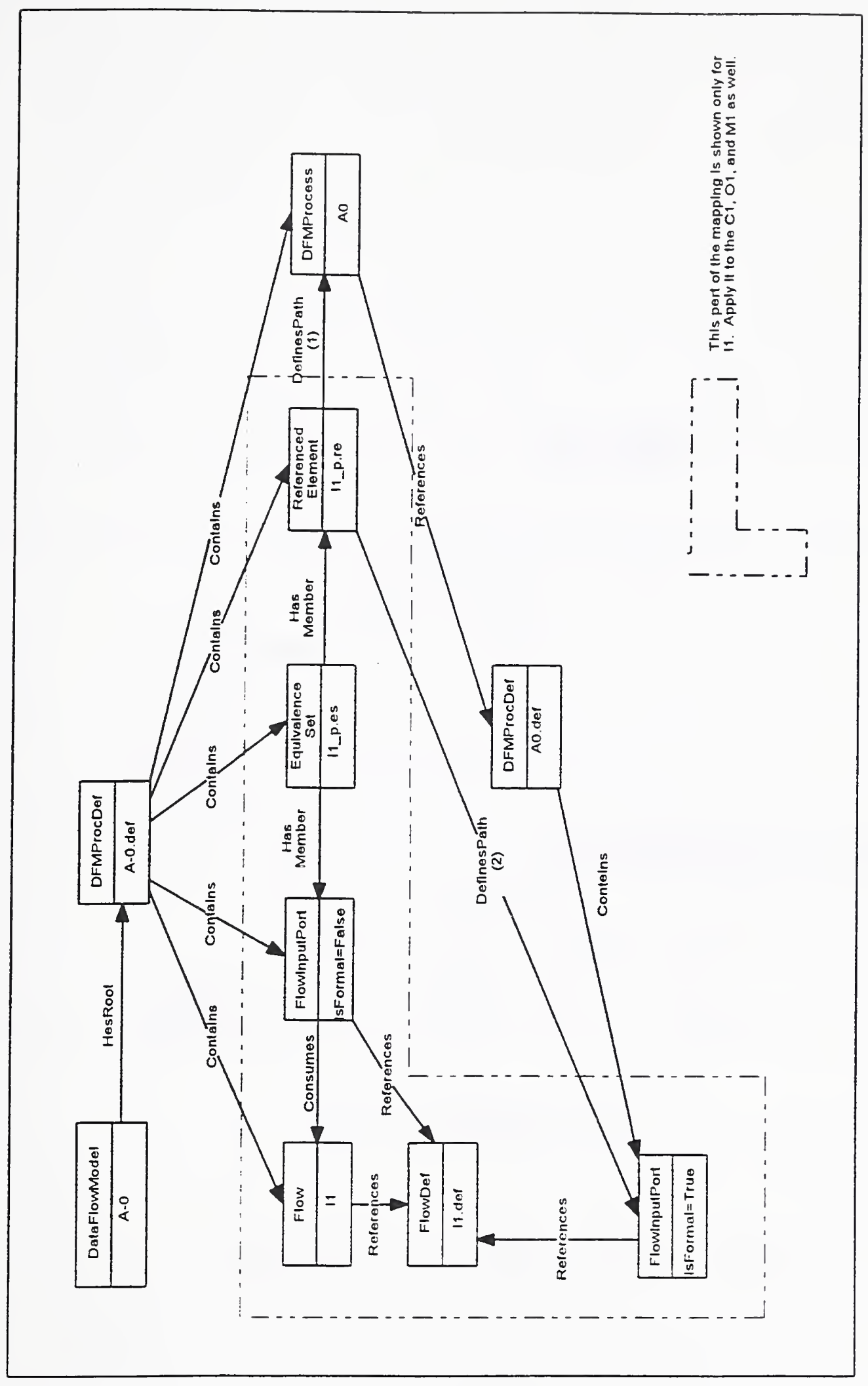

Figure 3-2: CDIF Mapping of A-O Diagram 


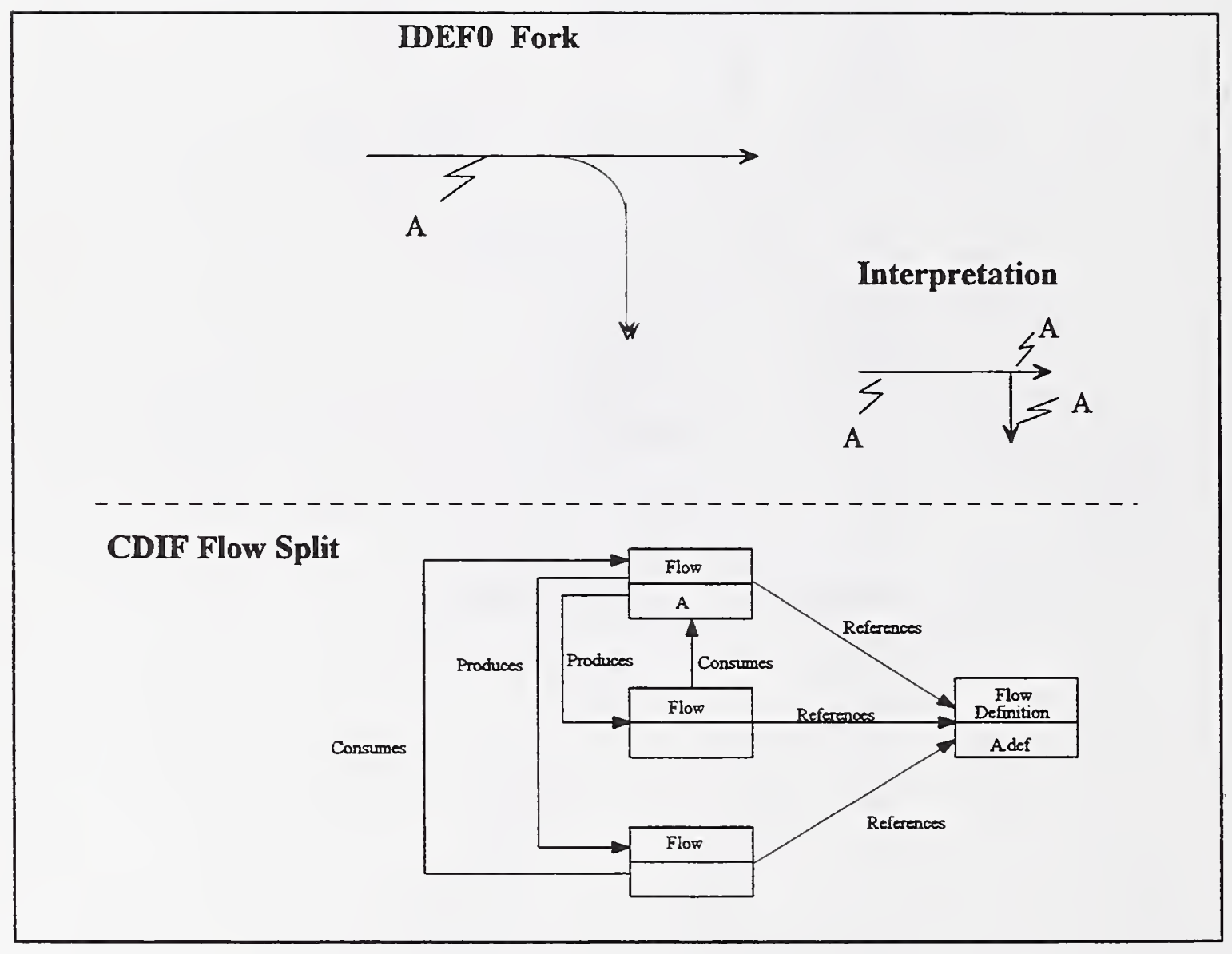

Figure 4: "A Copy" Fork and its CDIF Mapping 


\section{IDEF0 Fork}
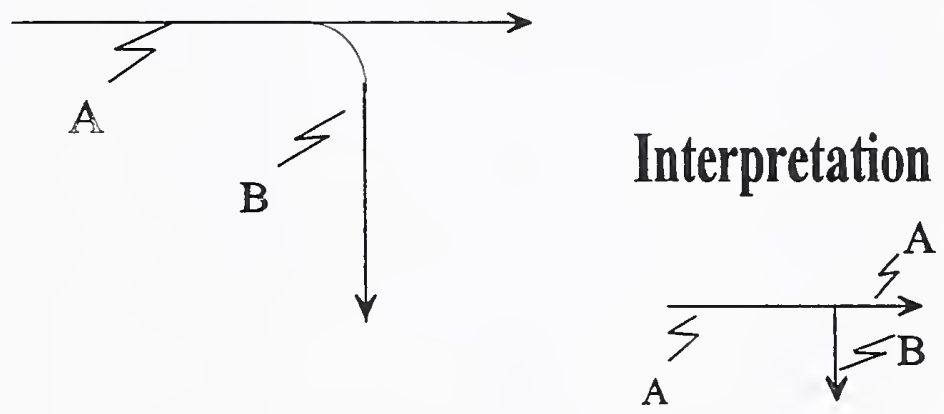

Figure 5: "A Portion" Fork 


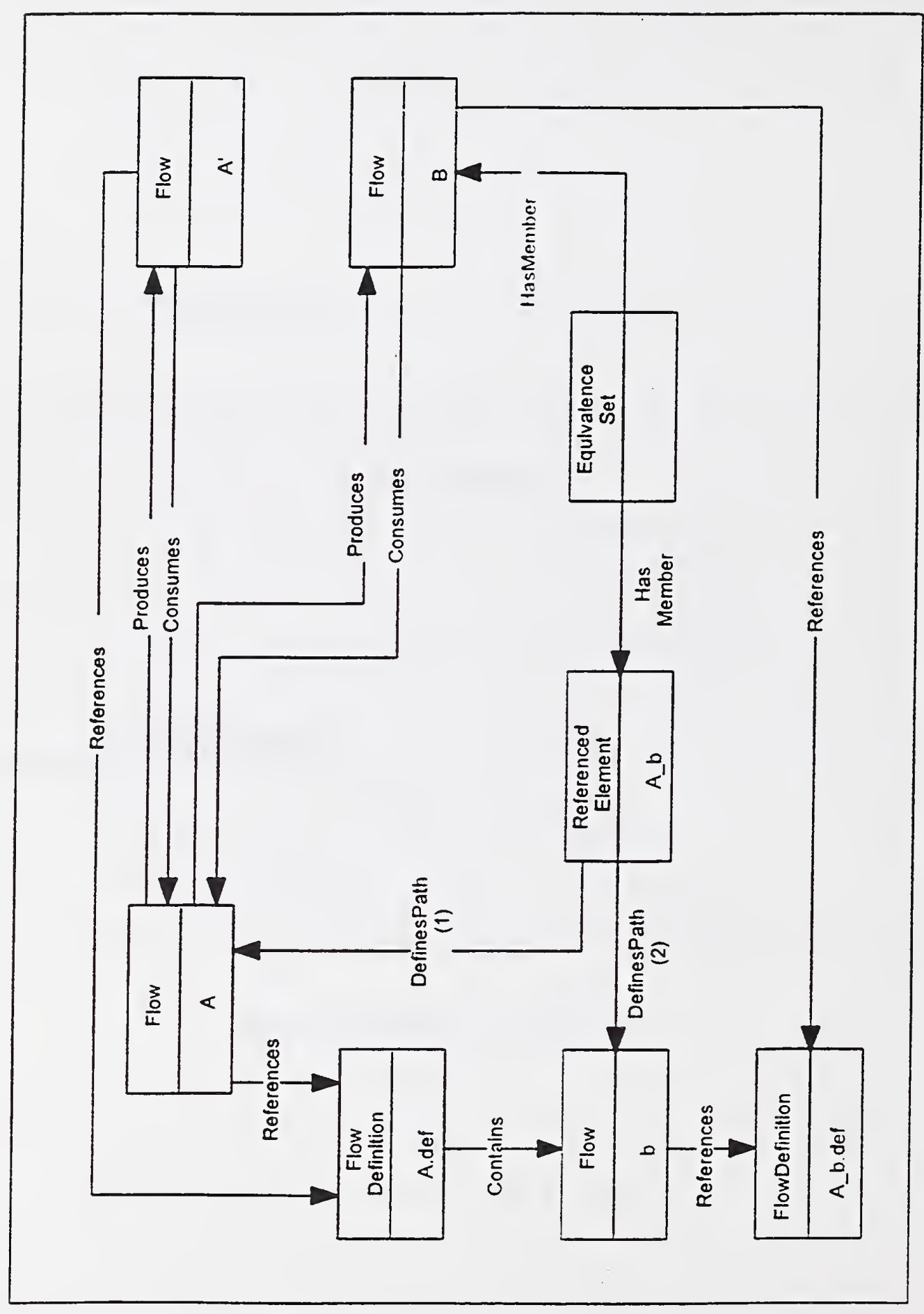

Figure 5-2: CDIF Mapping of "A Portion" Fork 


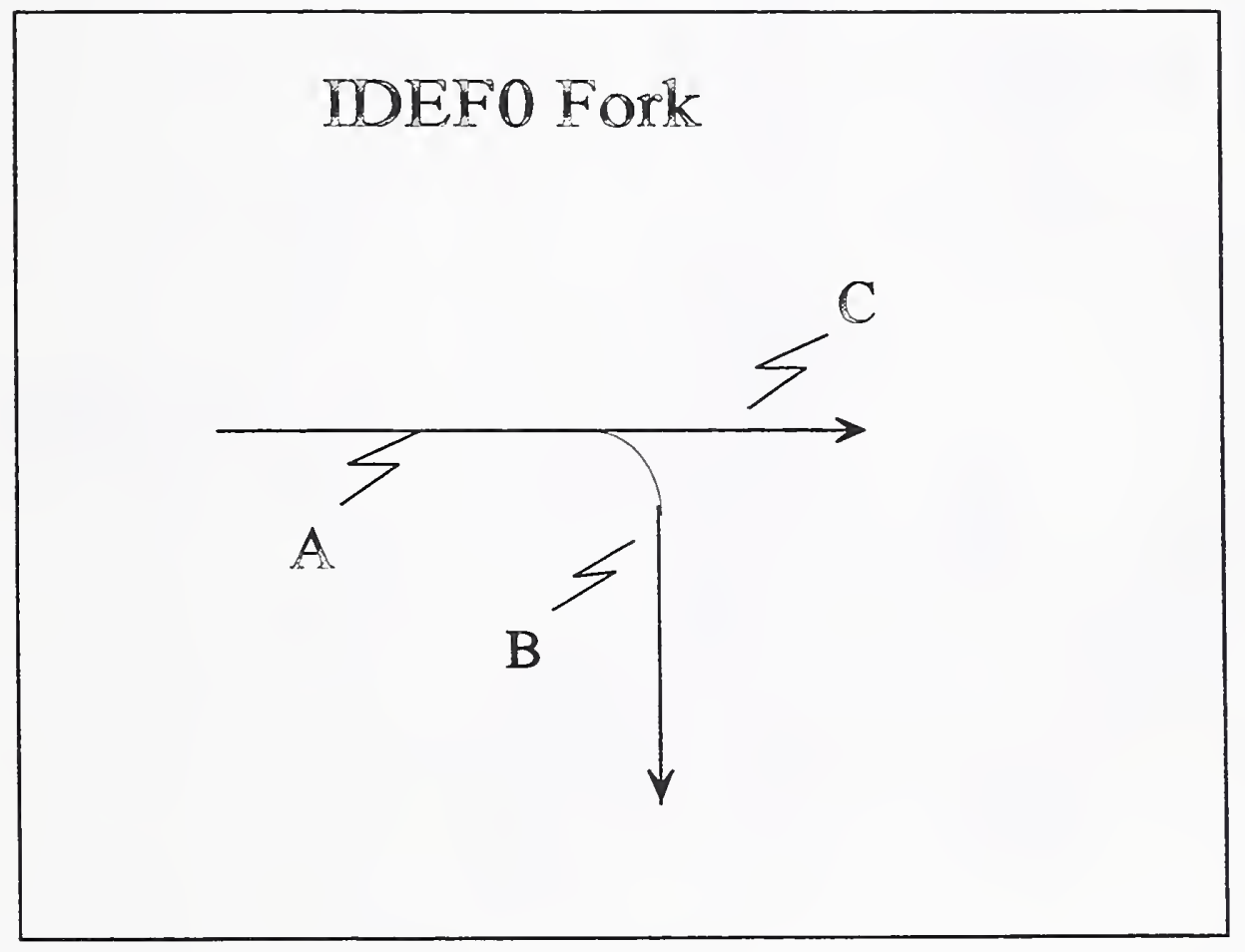

Figure 6: "The Sum" Fork 


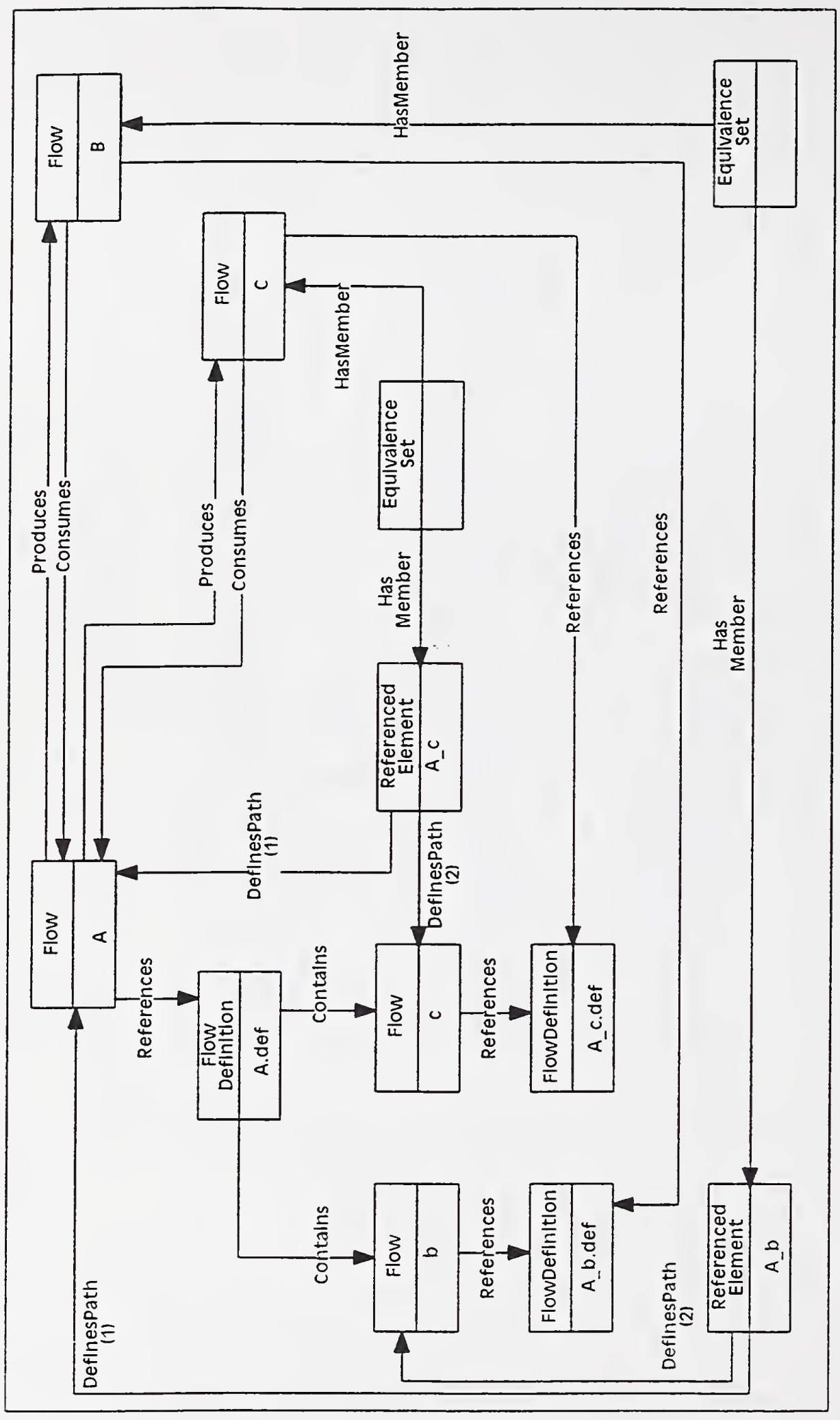

Figure 6-2: CDIF Mapping of "The Sum" Fork 


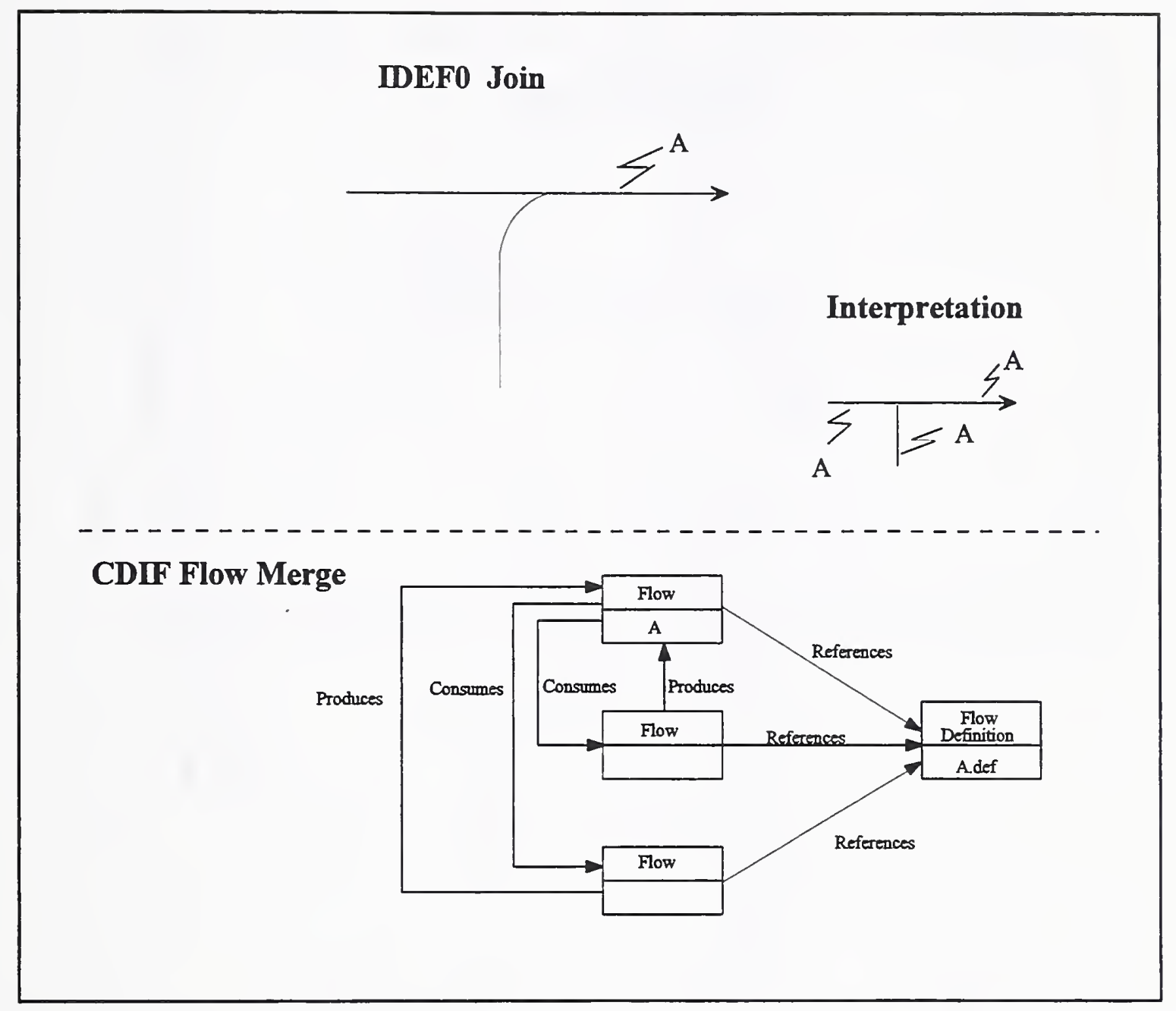

Figure 7: "A Copy" Join and its CDIF Mapping 


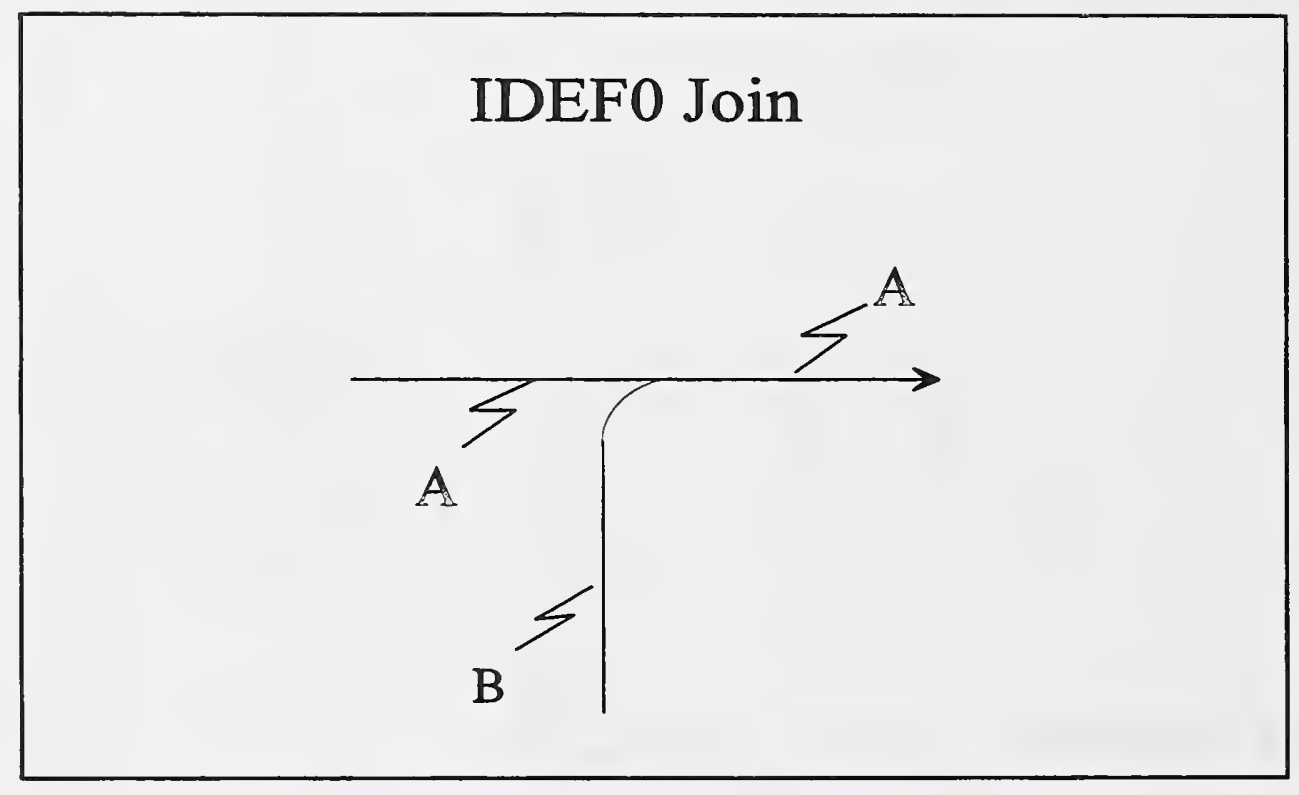

Figure 8: "A Portion" Join 


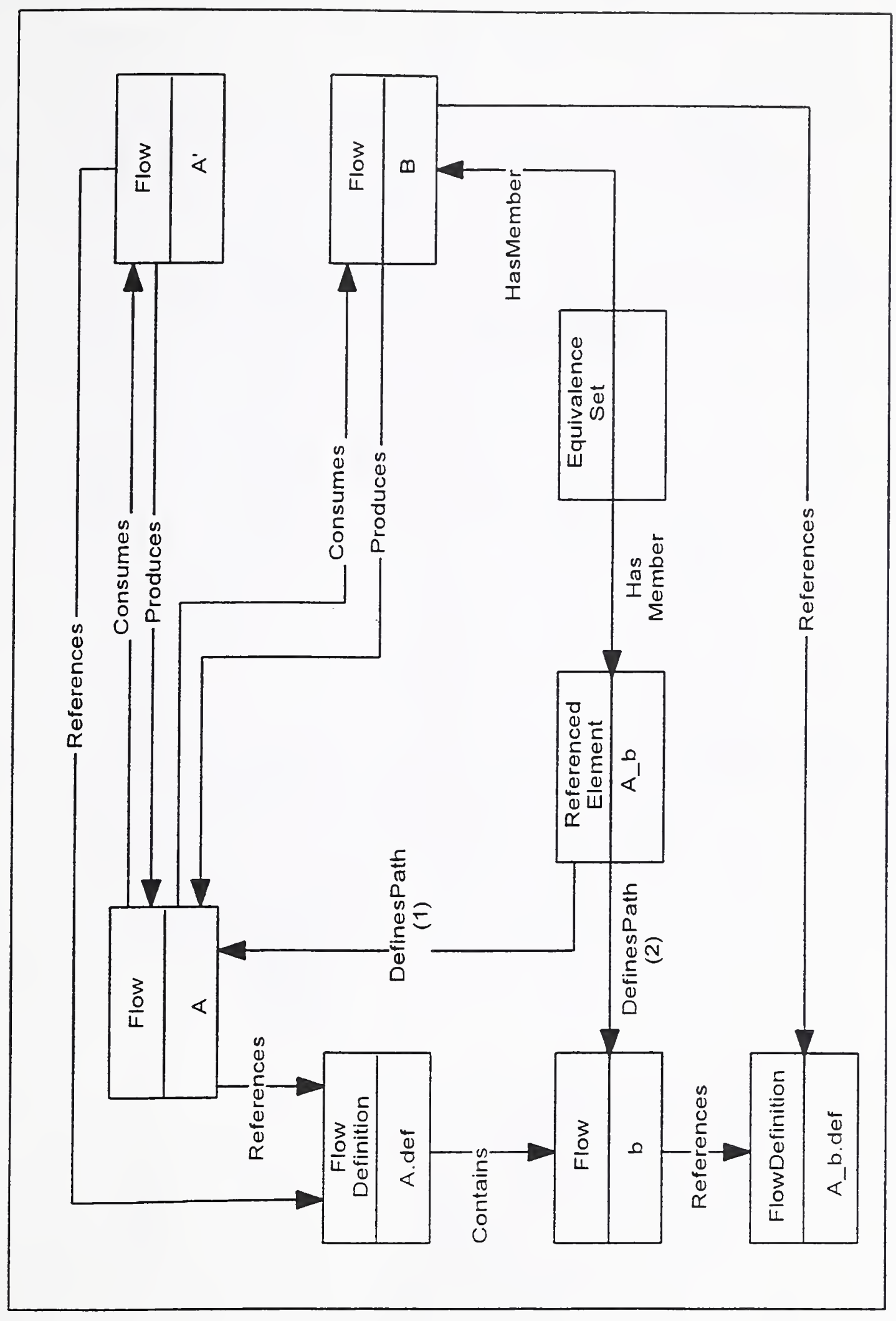

Figure 8-2: CDIF Mapping of "A Portion" Join 


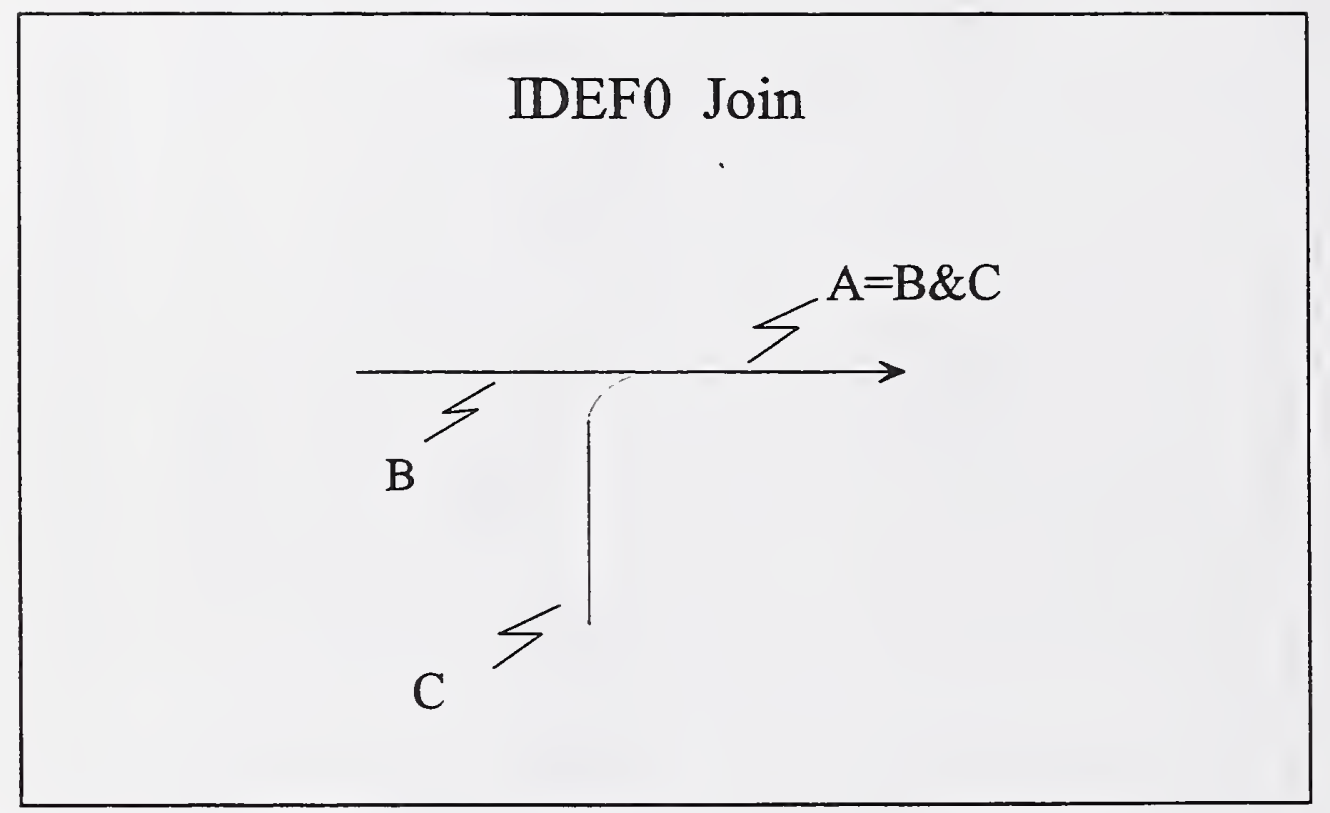

Figure 9: "The Sum" Join 


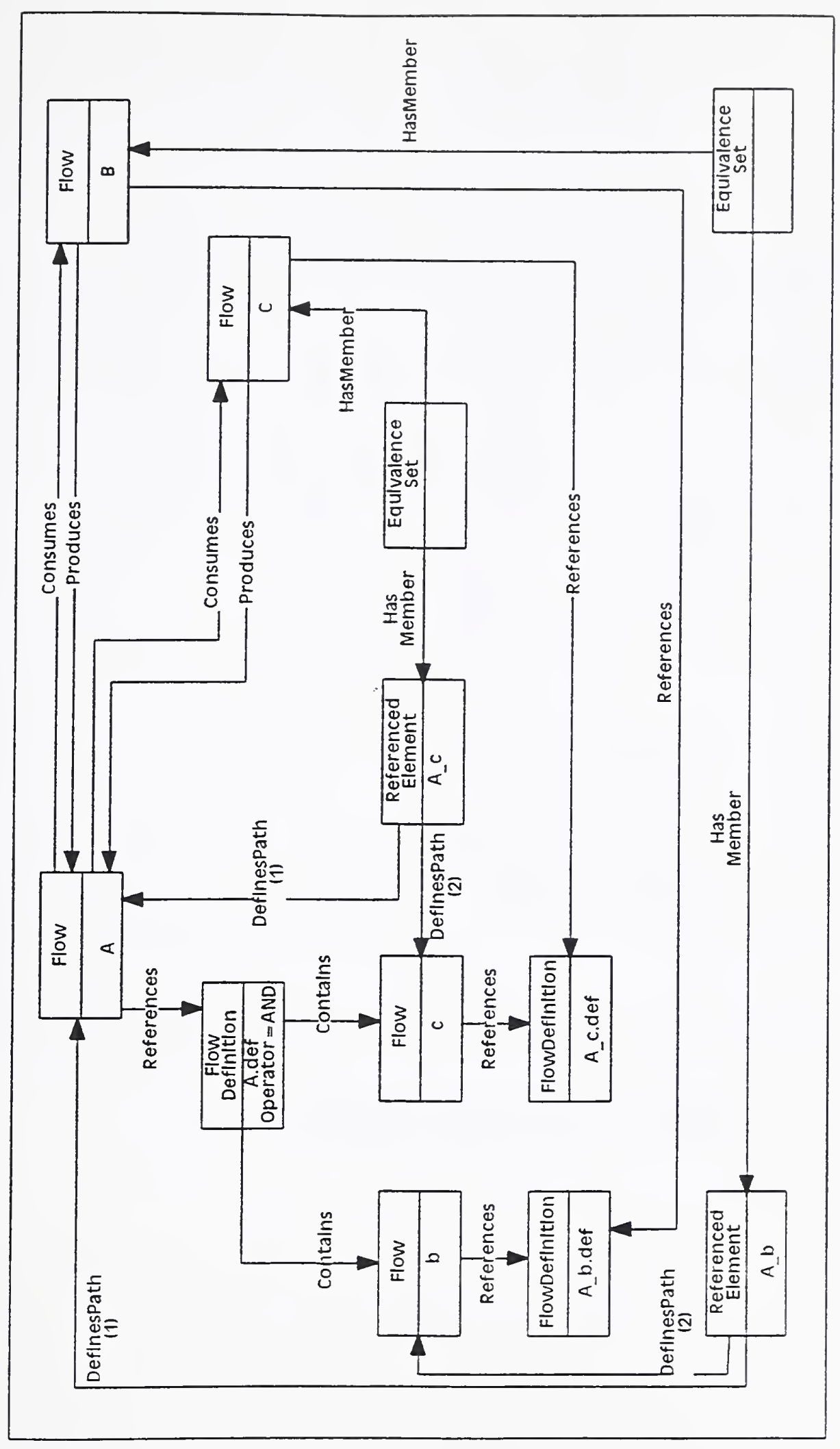

Figure 9-2: CDIF Mapping of "The Sum" Join 


\section{IDEF0 Call}
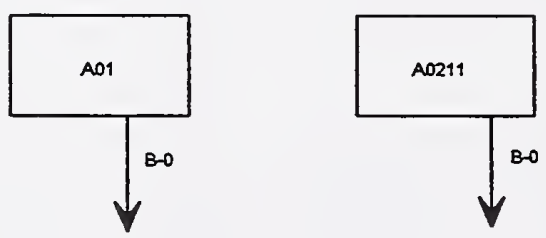

B- 0

\section{CDIF}

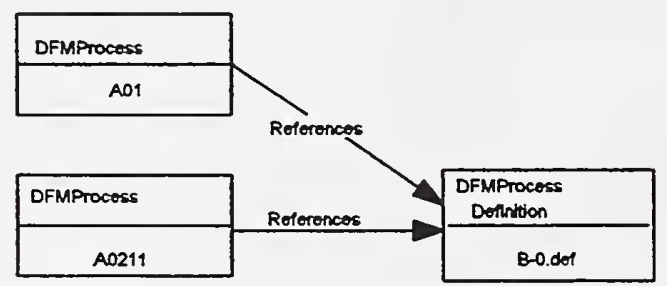

Figure 10: "Another Data Model" Call 


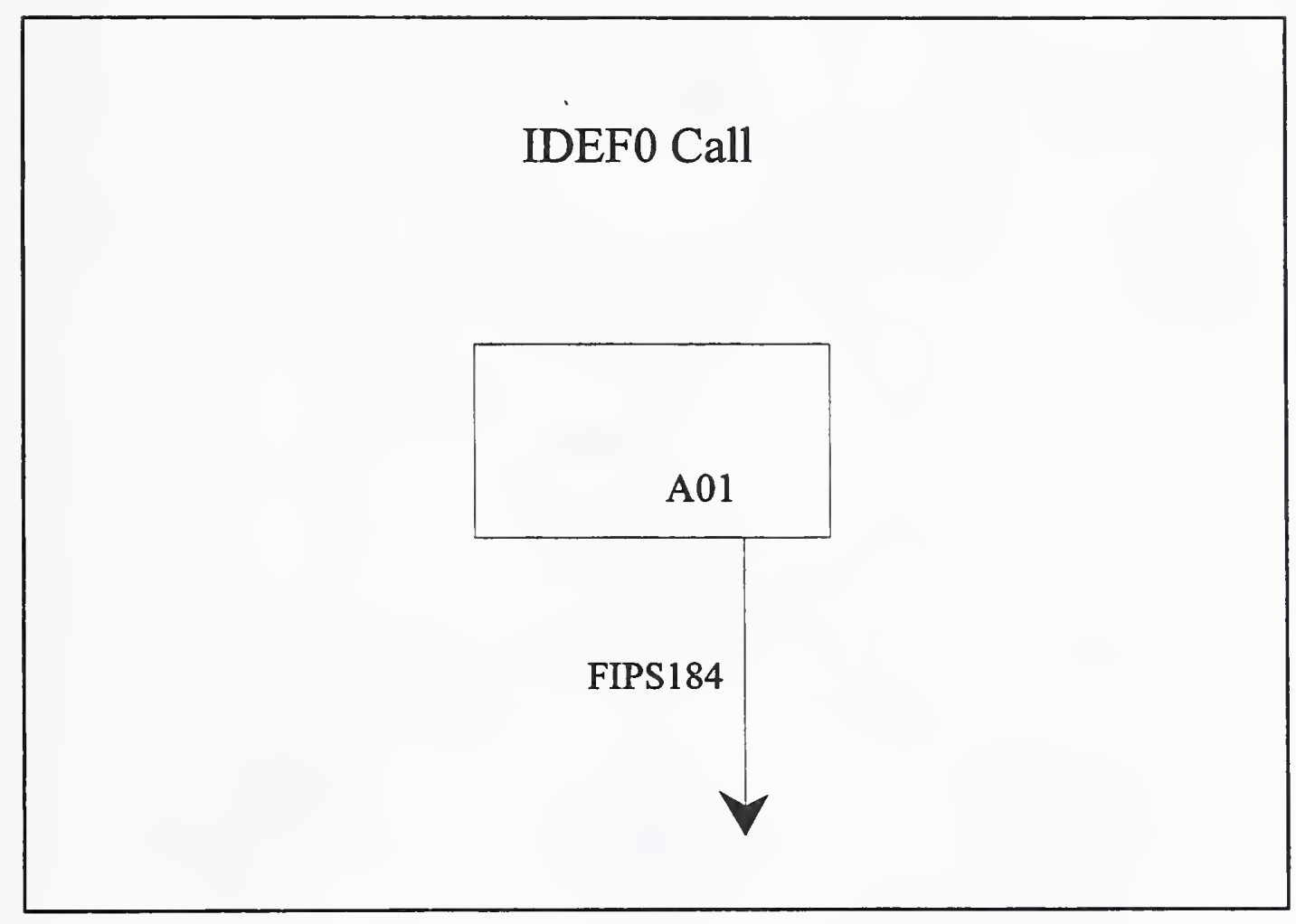

Figure 11: "A Document" Call 


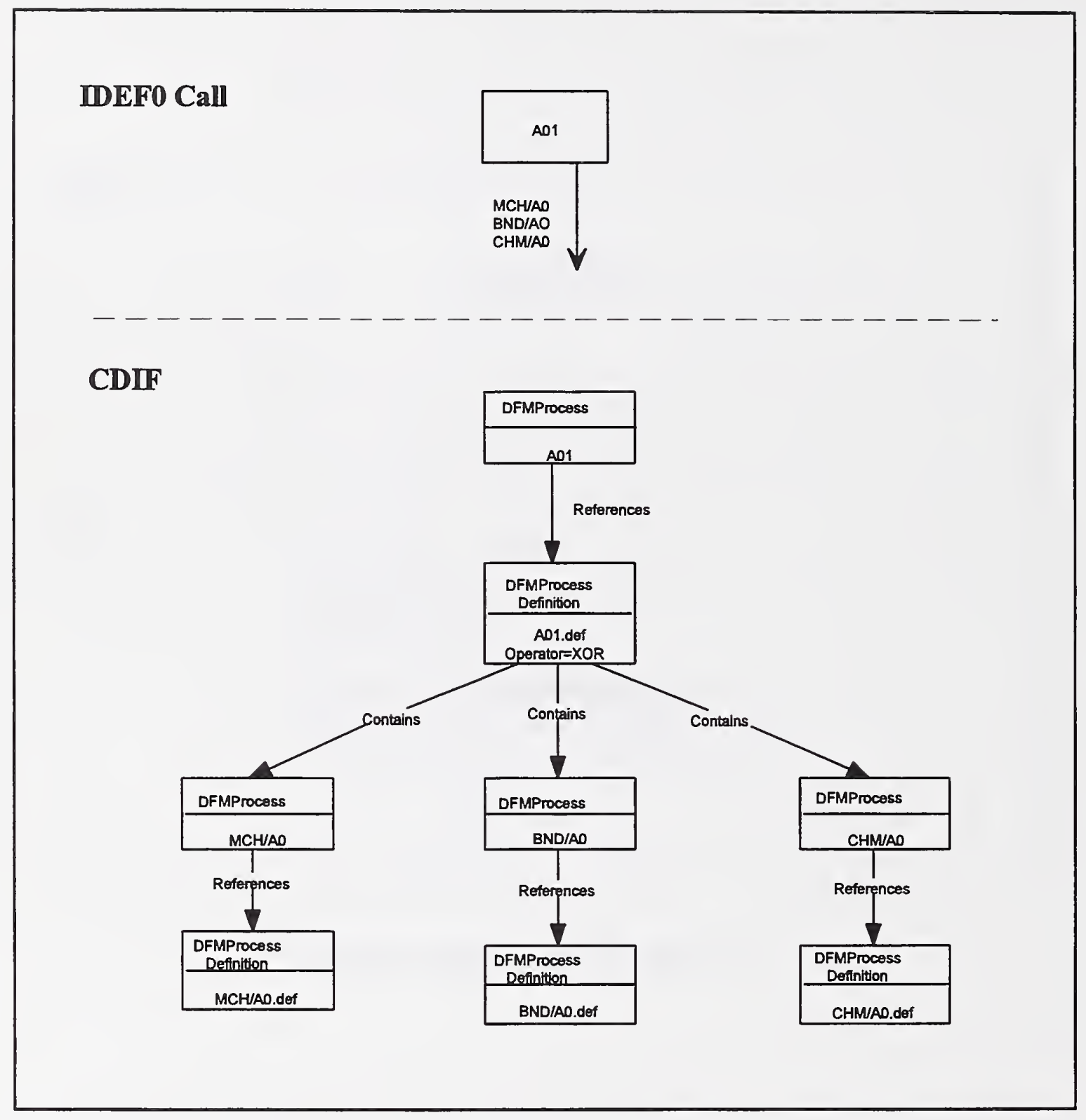

Figure 12: SBT Call 


\section{IDEF0 Call}

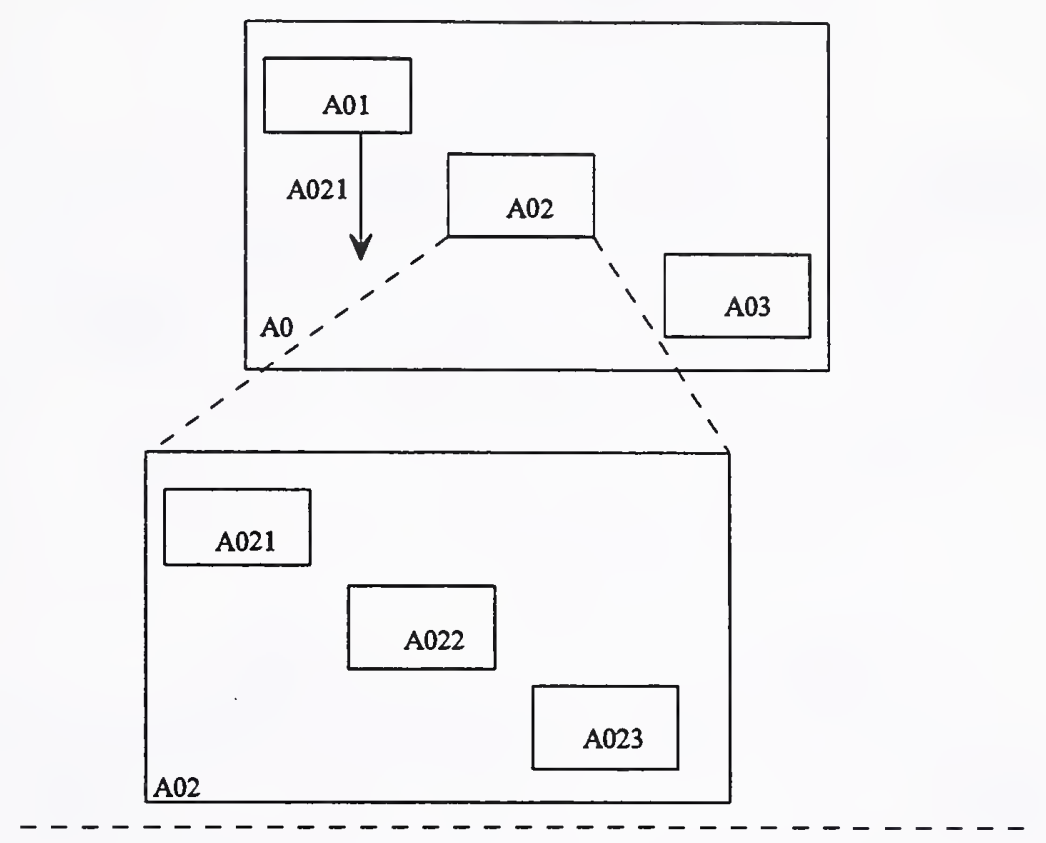

\section{$\mathrm{CDIF}$}

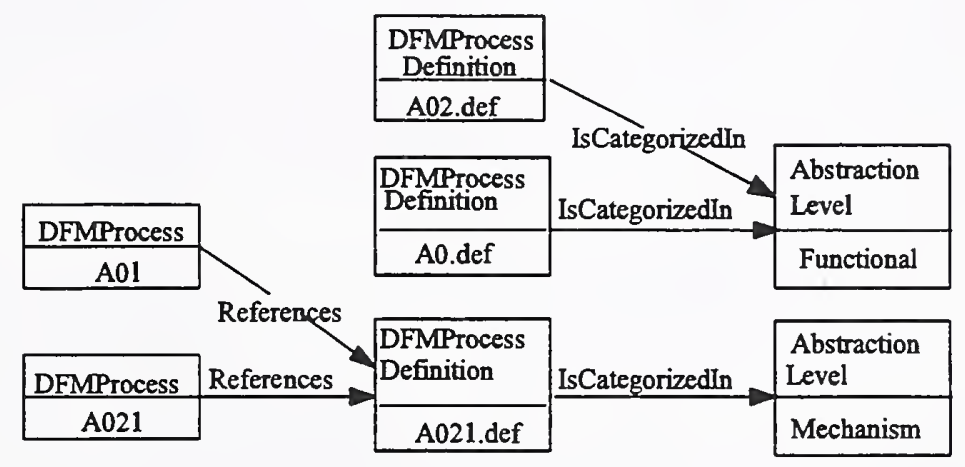

Figure 13: A Drop in the Level of Abstraction Call 


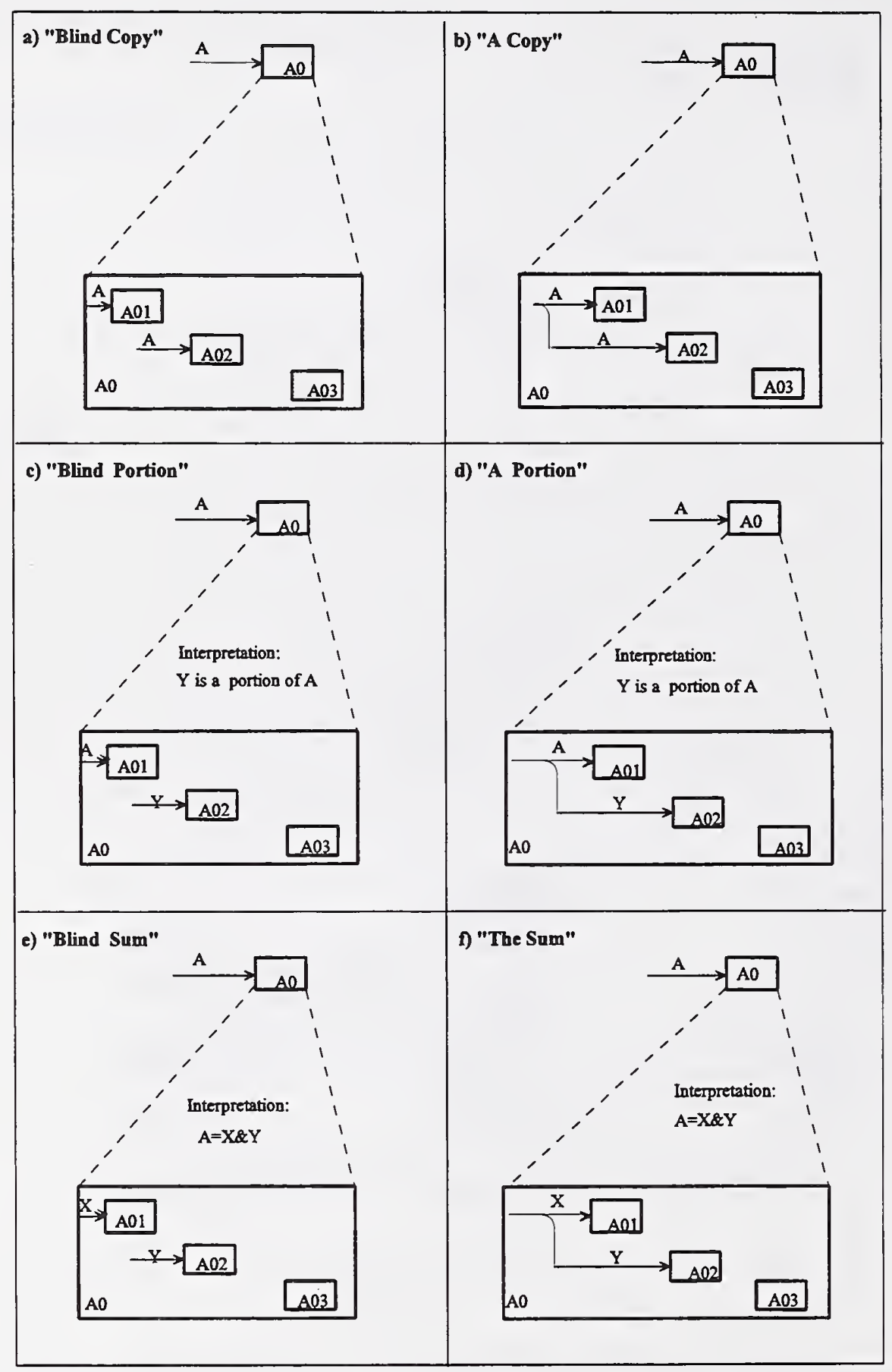

Figure 14: Types of Flow Decomposition 


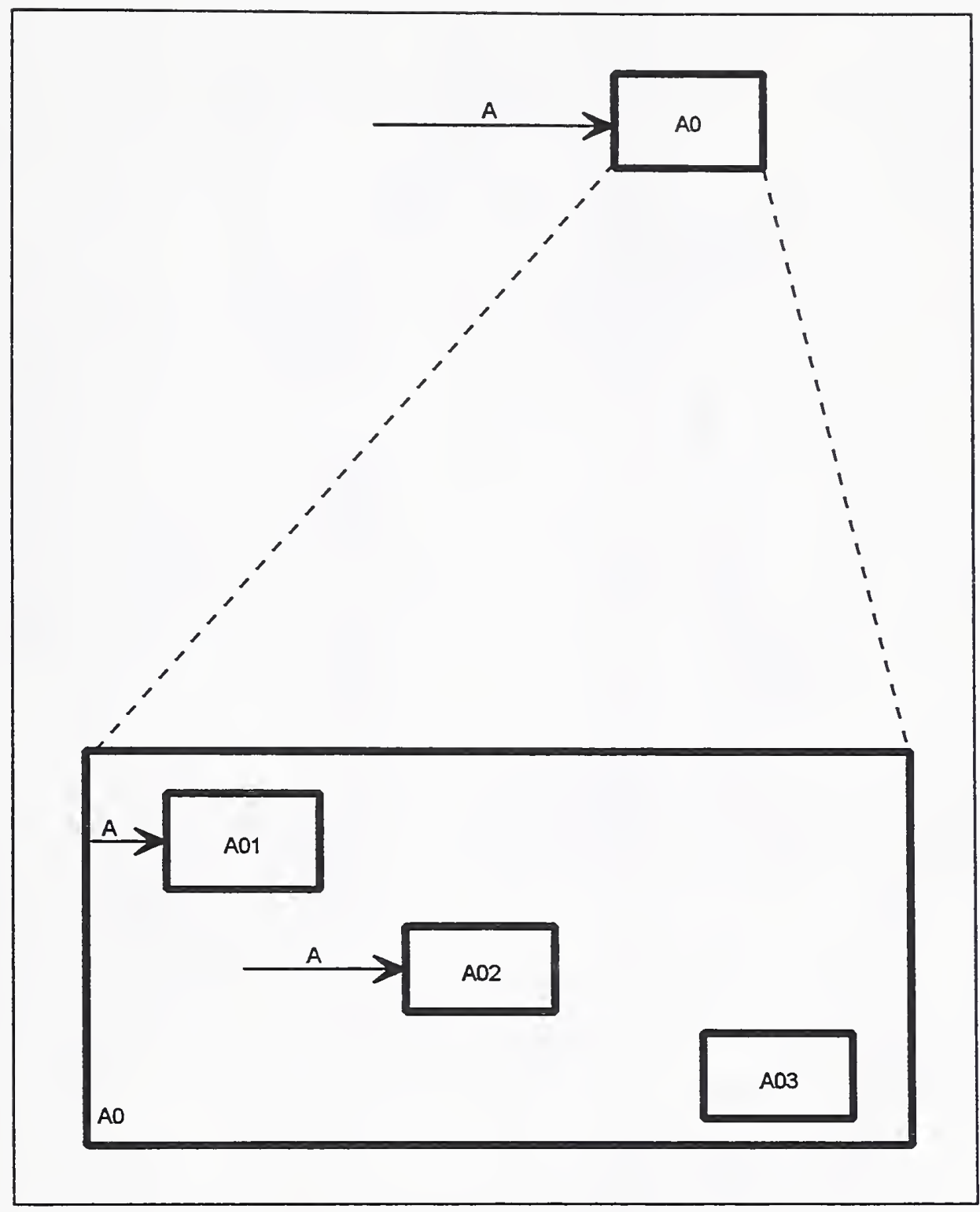

Figure 15: "Blind Copy" Decomposition 


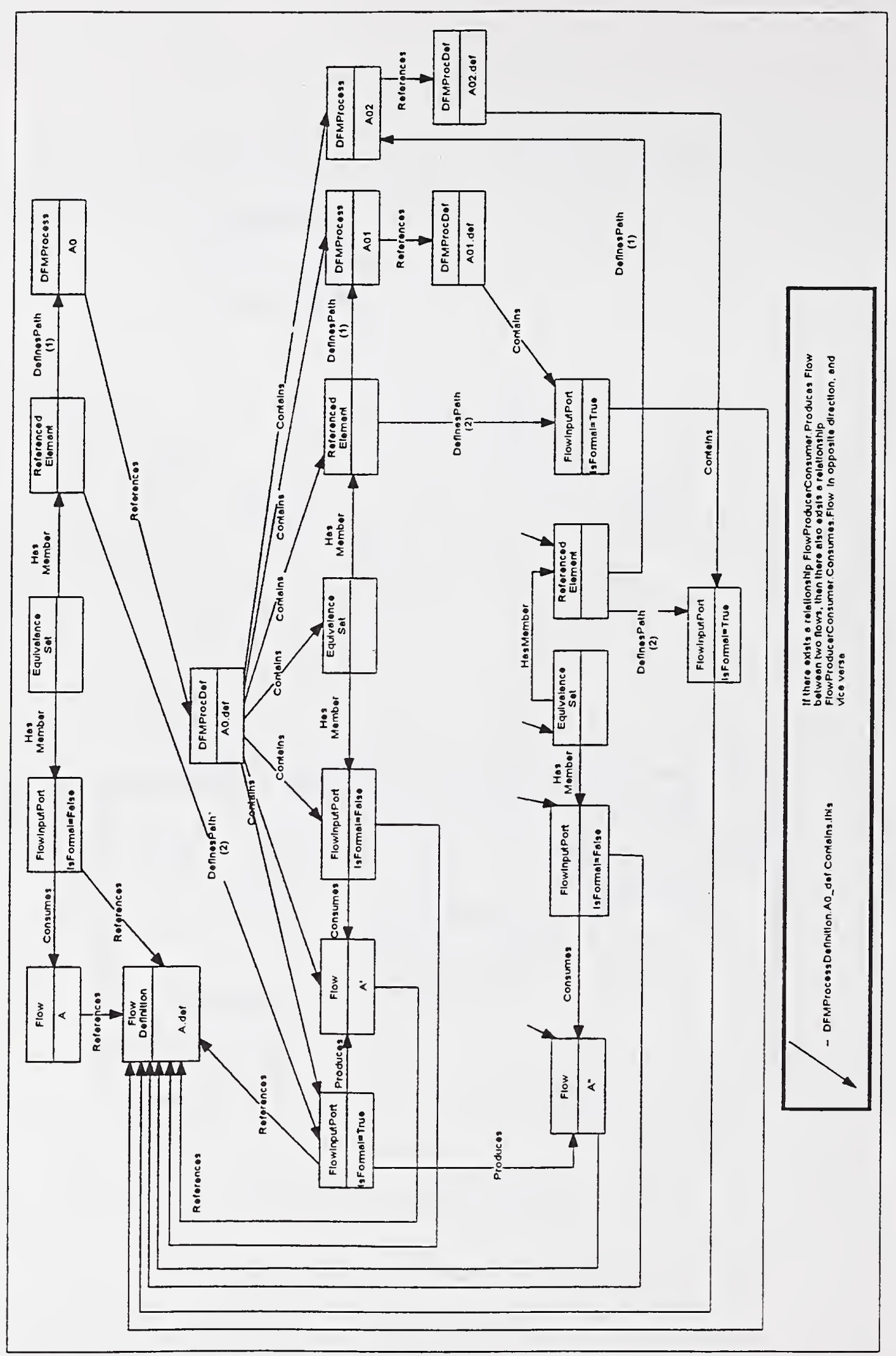

Figure 15-2: CDIF Mapping of the "Blind Copy" Decomposition 


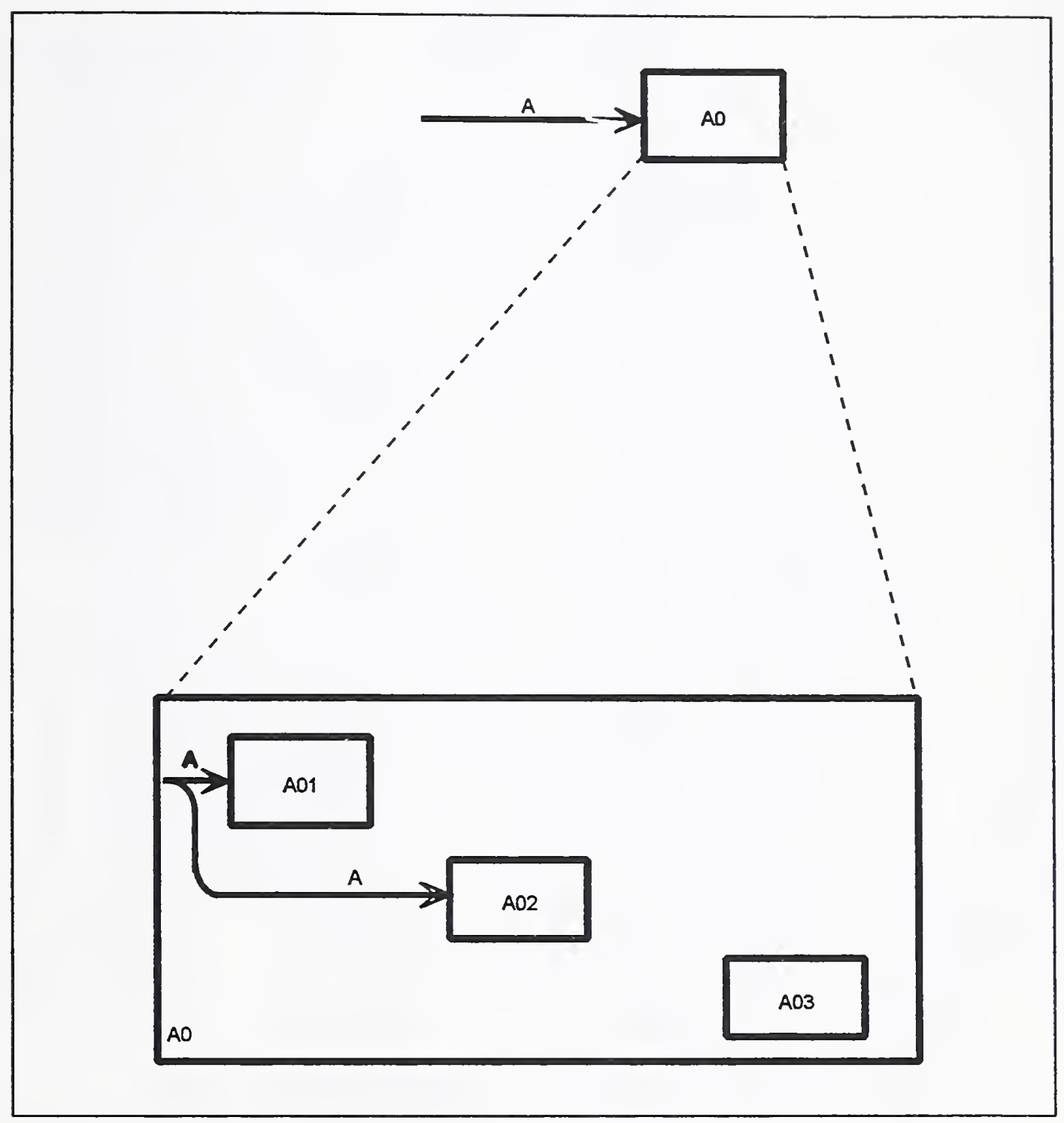

Figure 16: "A Copy" Decomposition 


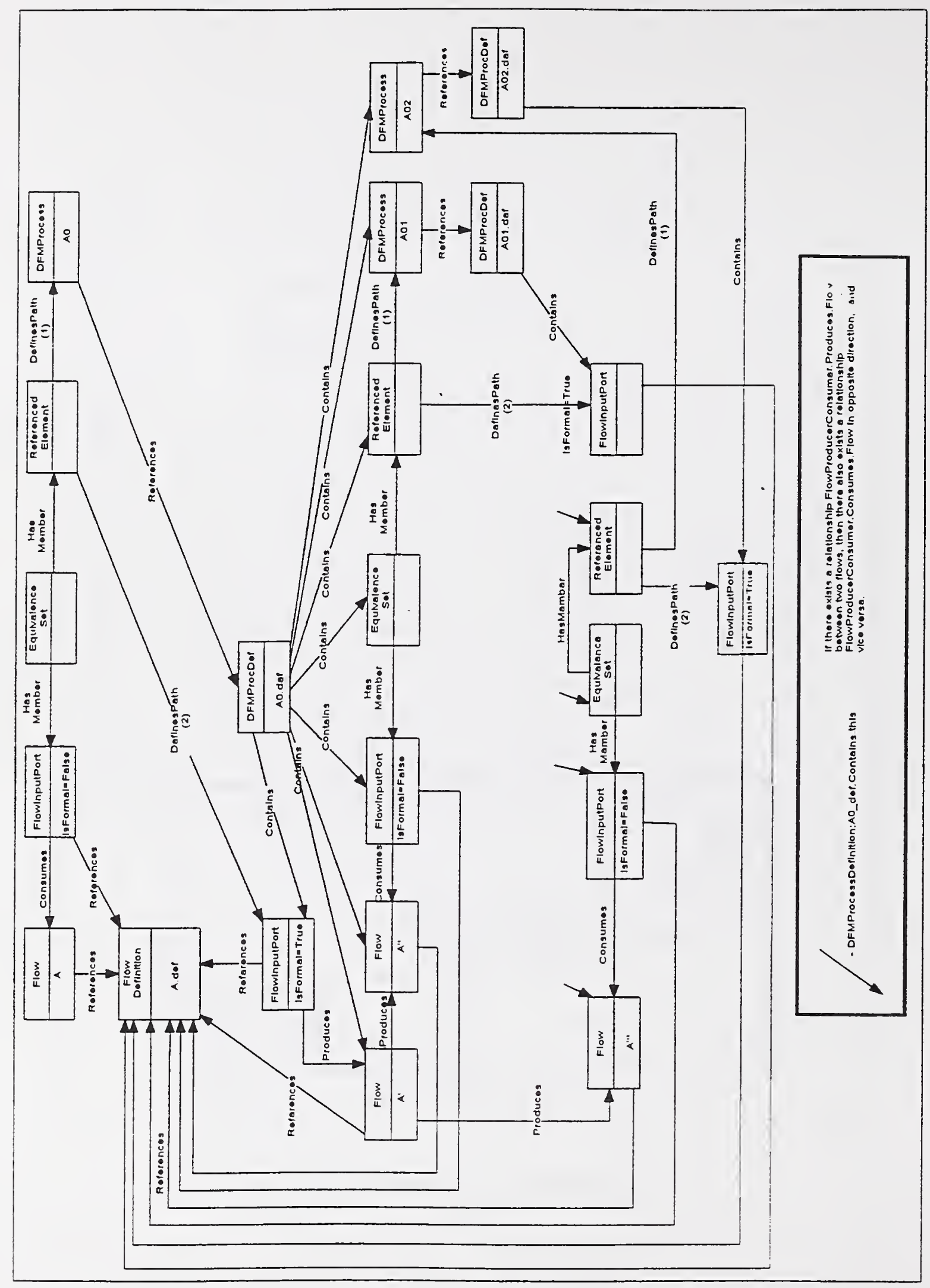

Figure 16-2: CDIF Mapping of the "Copy" Decomposition 


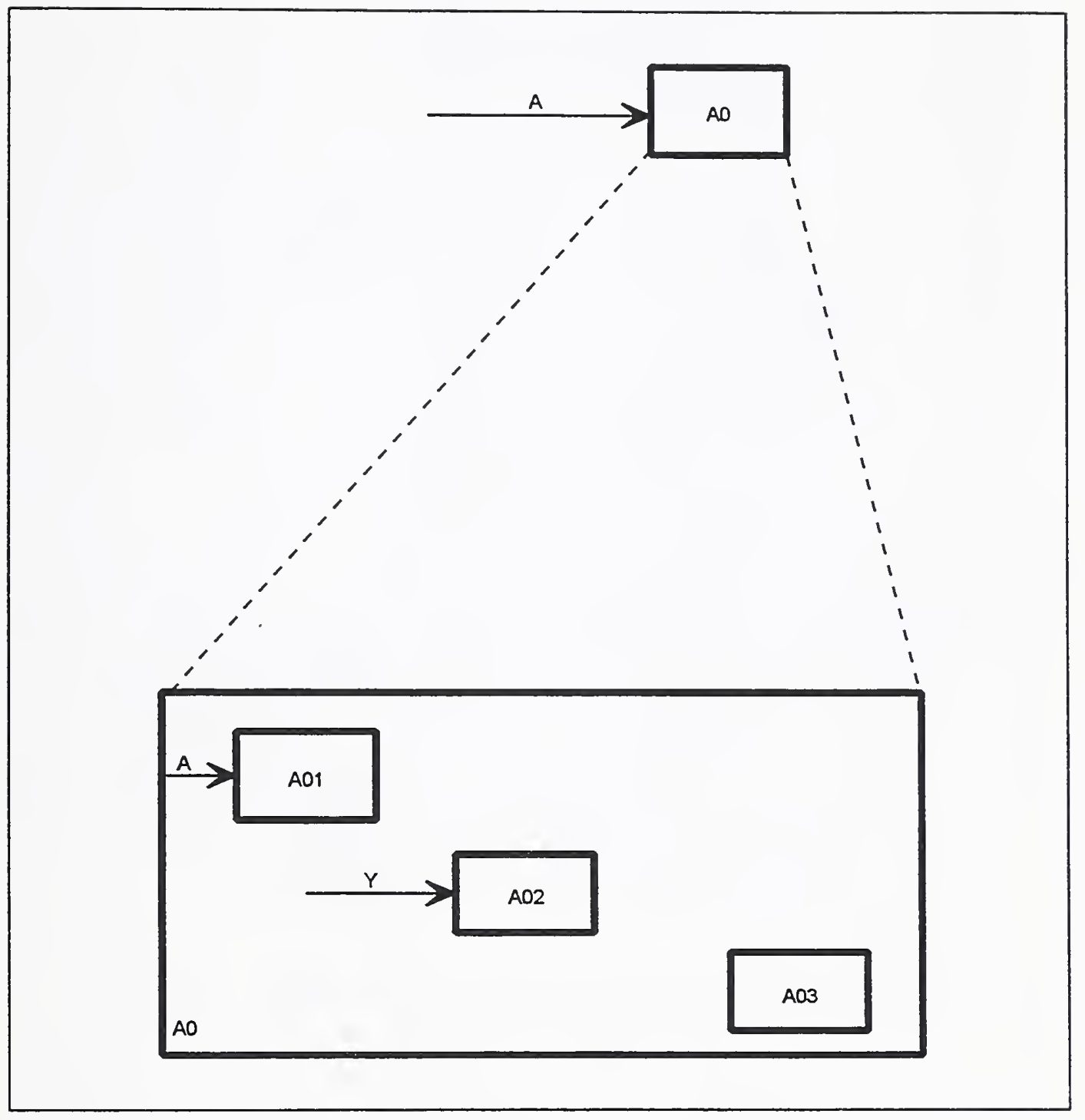

Figure 17: "Blind Portion" Decomposition 


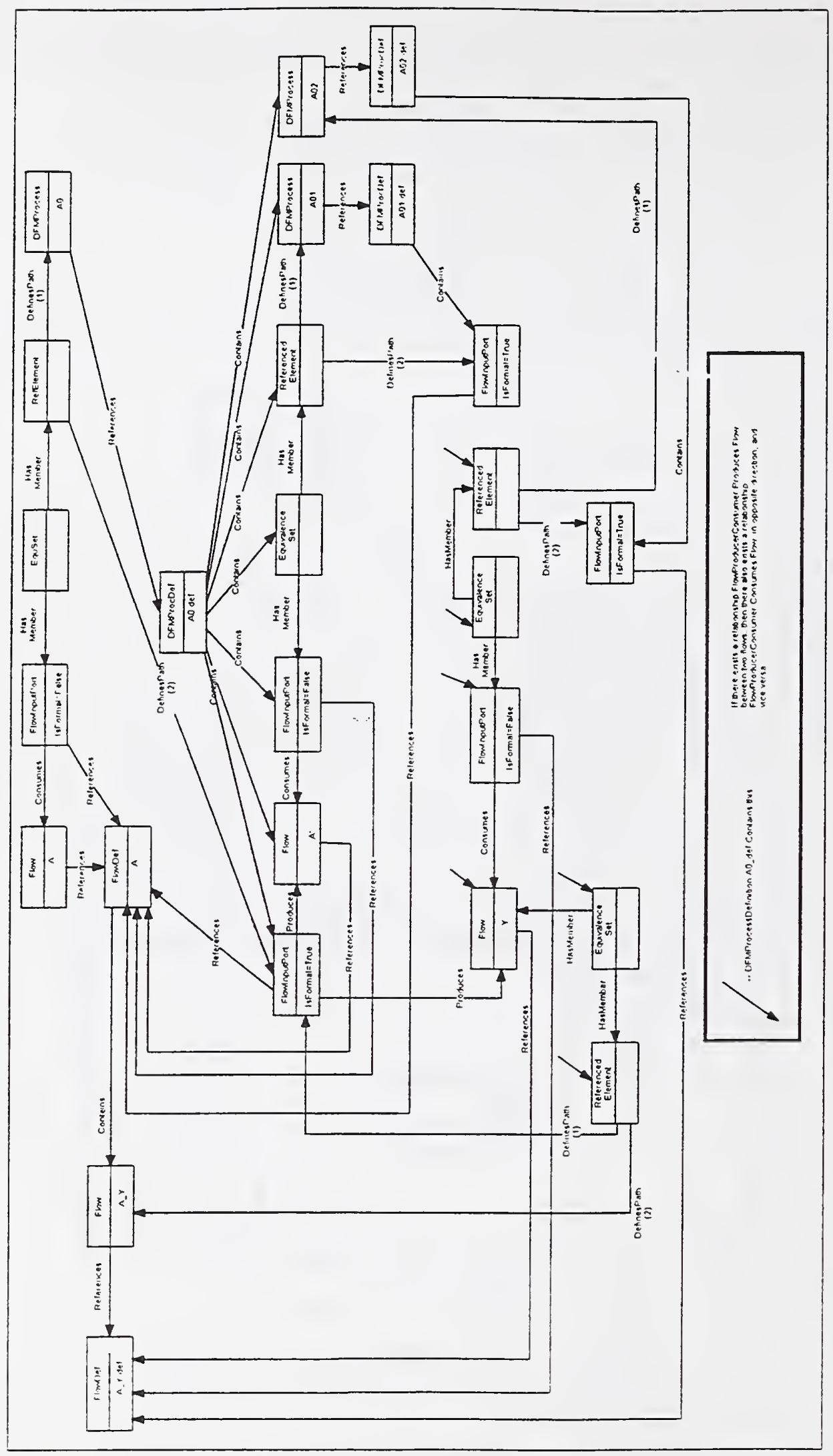

Figure 17-2: CDIF Mapping of the "Blind Portion" Decomposition 


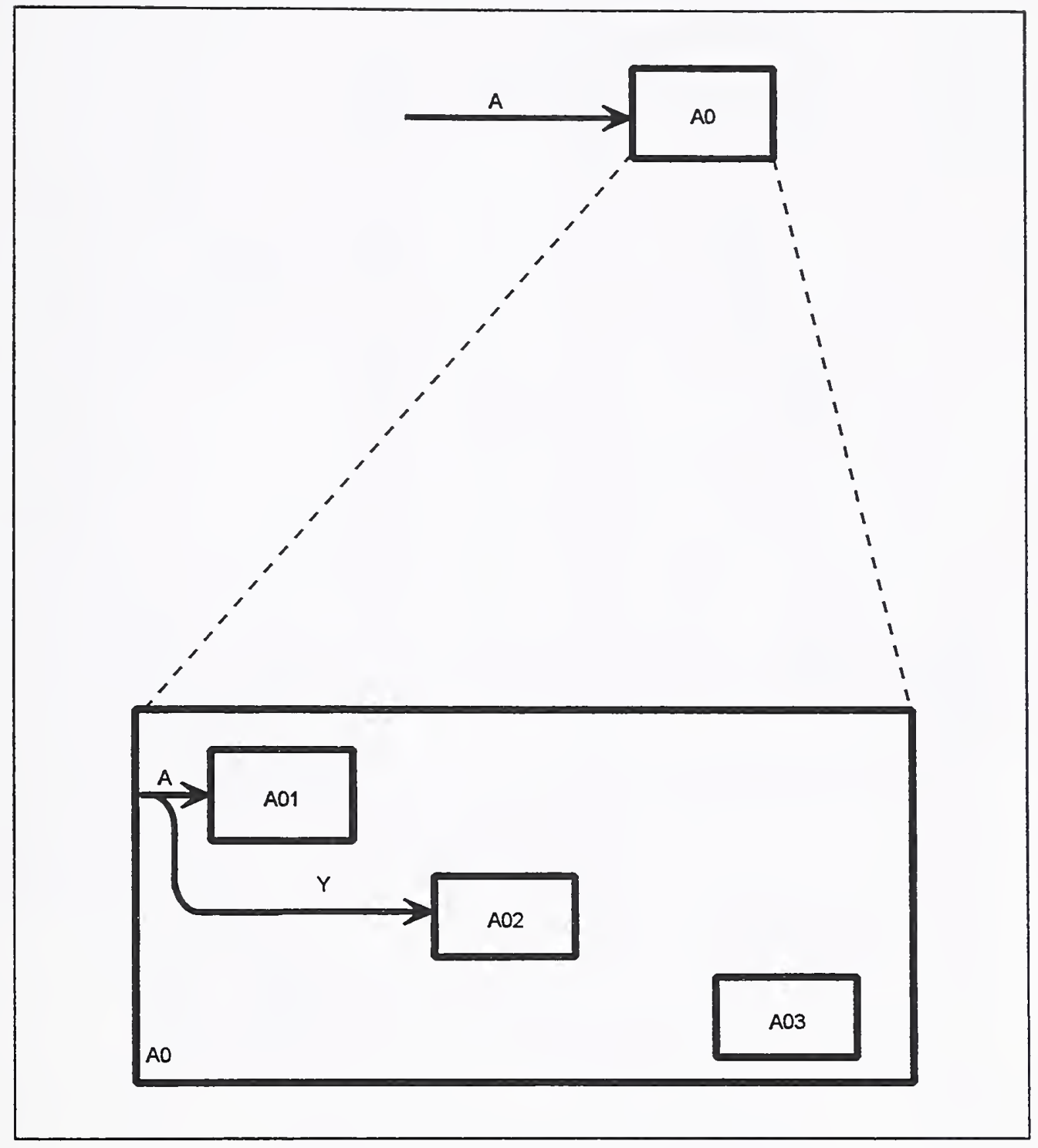

Figure 18: "A Portion" Decomposition 


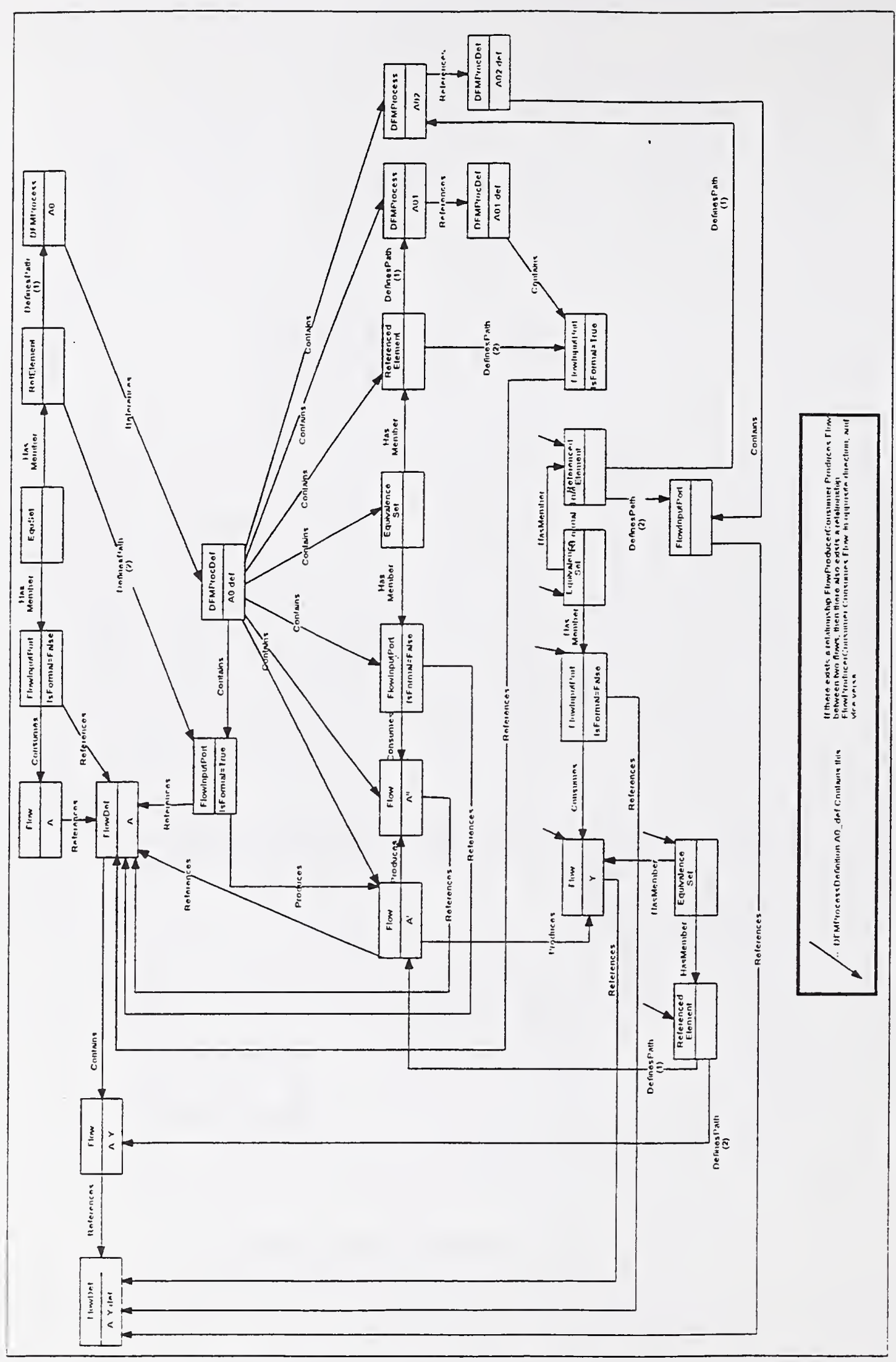

Figure 18-2: CDIF Mapping of the"Portion" Decomposition 


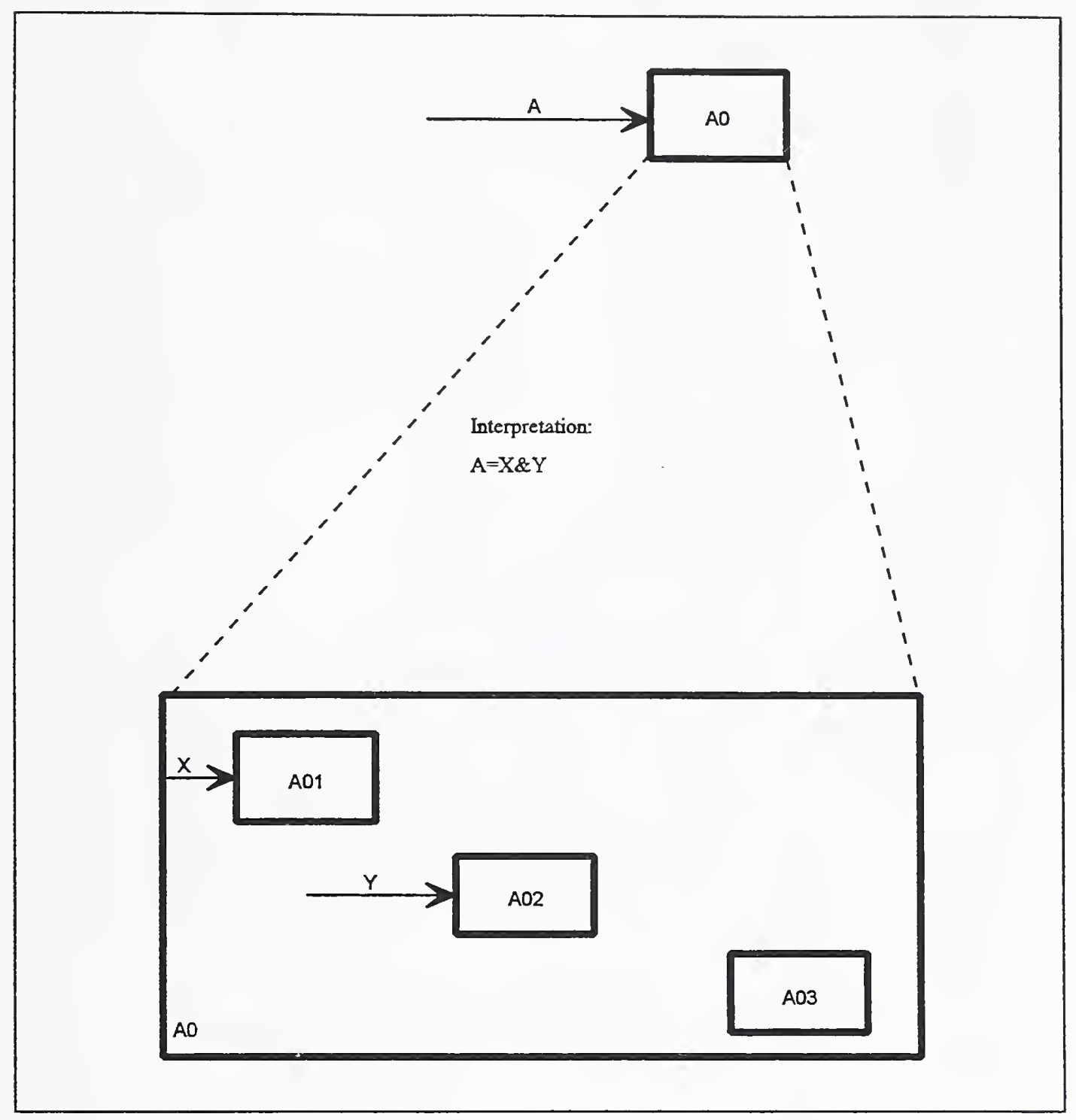

Figure 19: "Blind Sum" Decomposition 


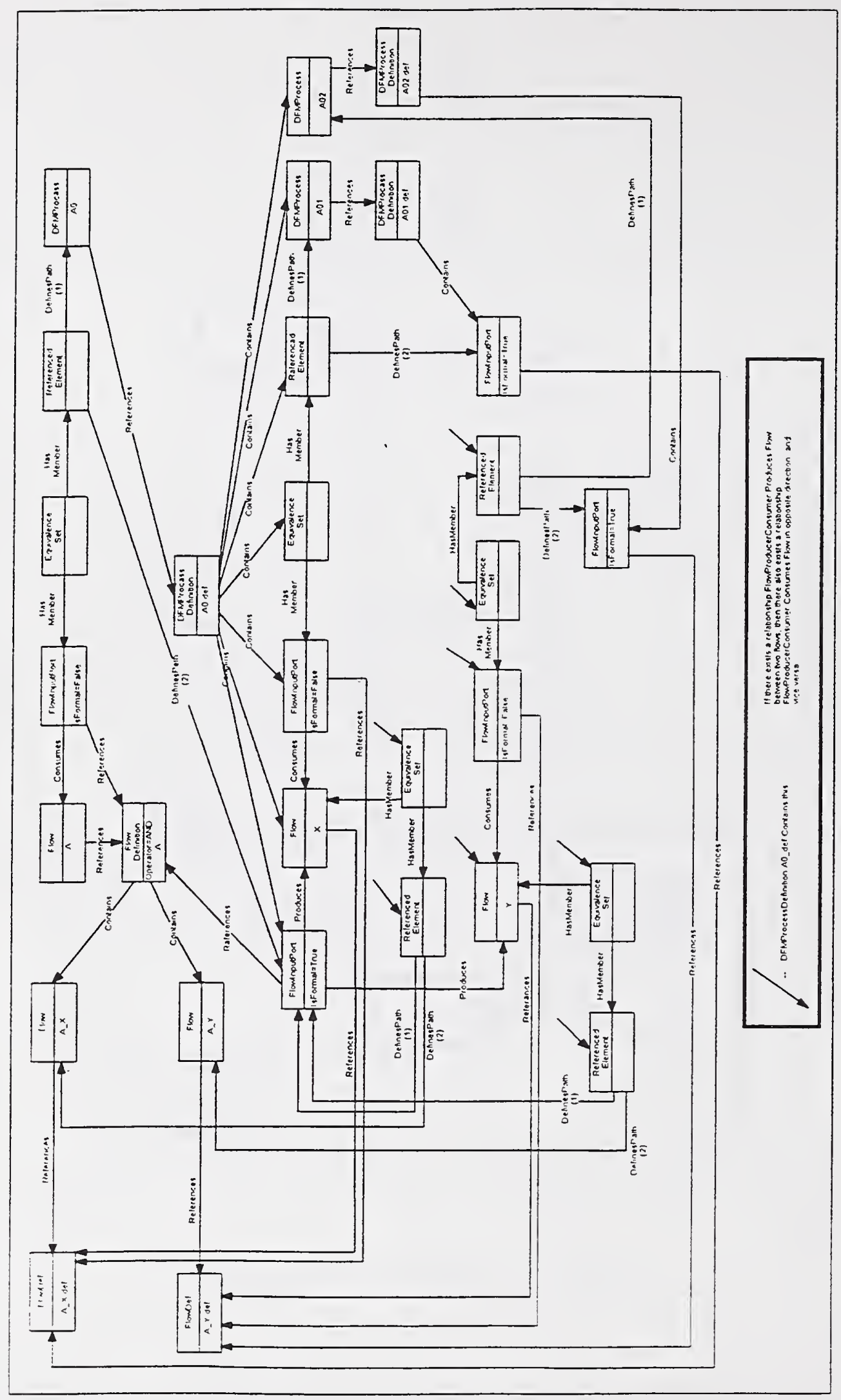

Figure 19-2: CDIF Mapping of the "Blind Sum" Decomposition 


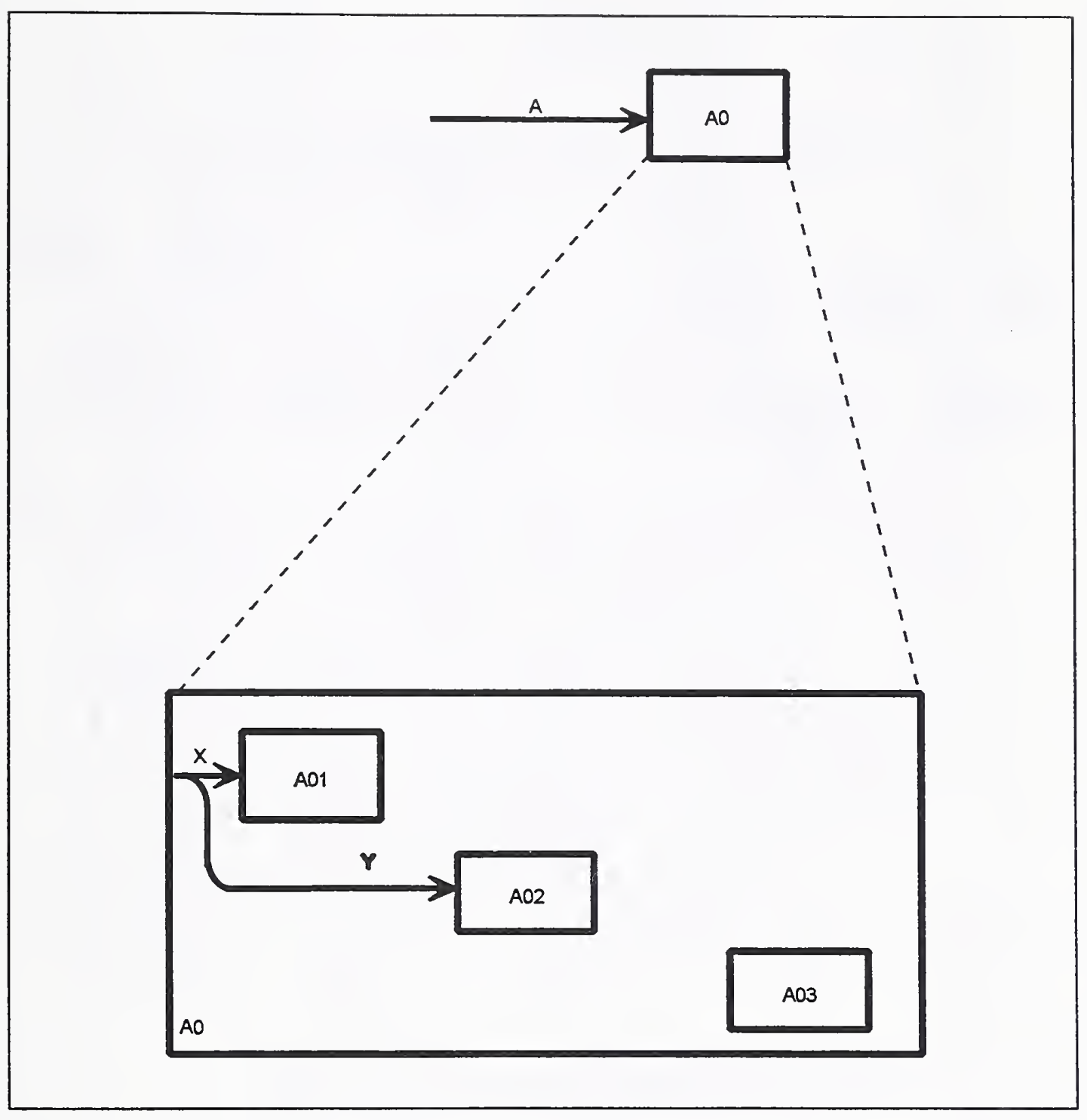

Figure 20: "The Sum" Decomposition 


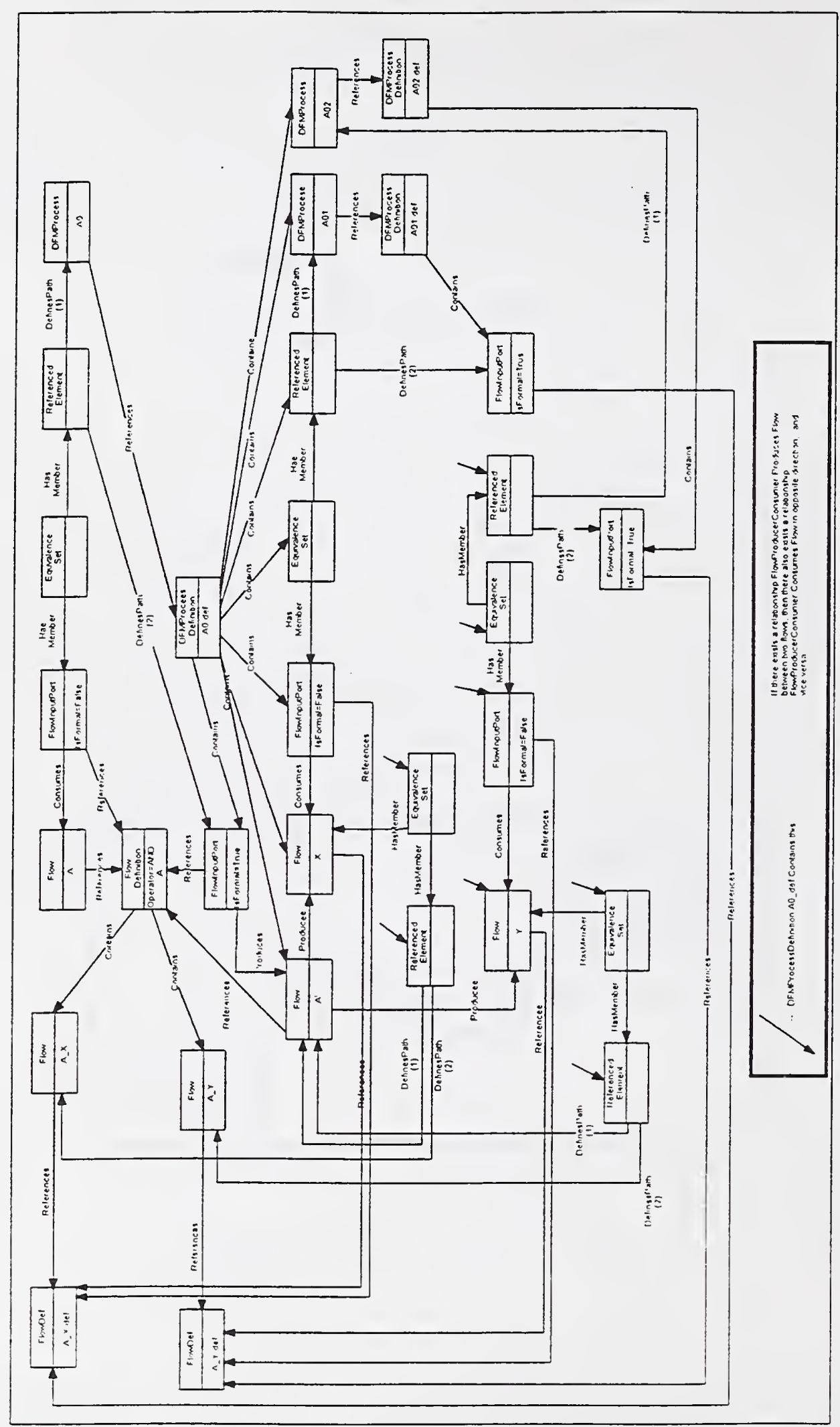

Figure 20-2: CDIF Mapping of the "Sum" Decomposition 


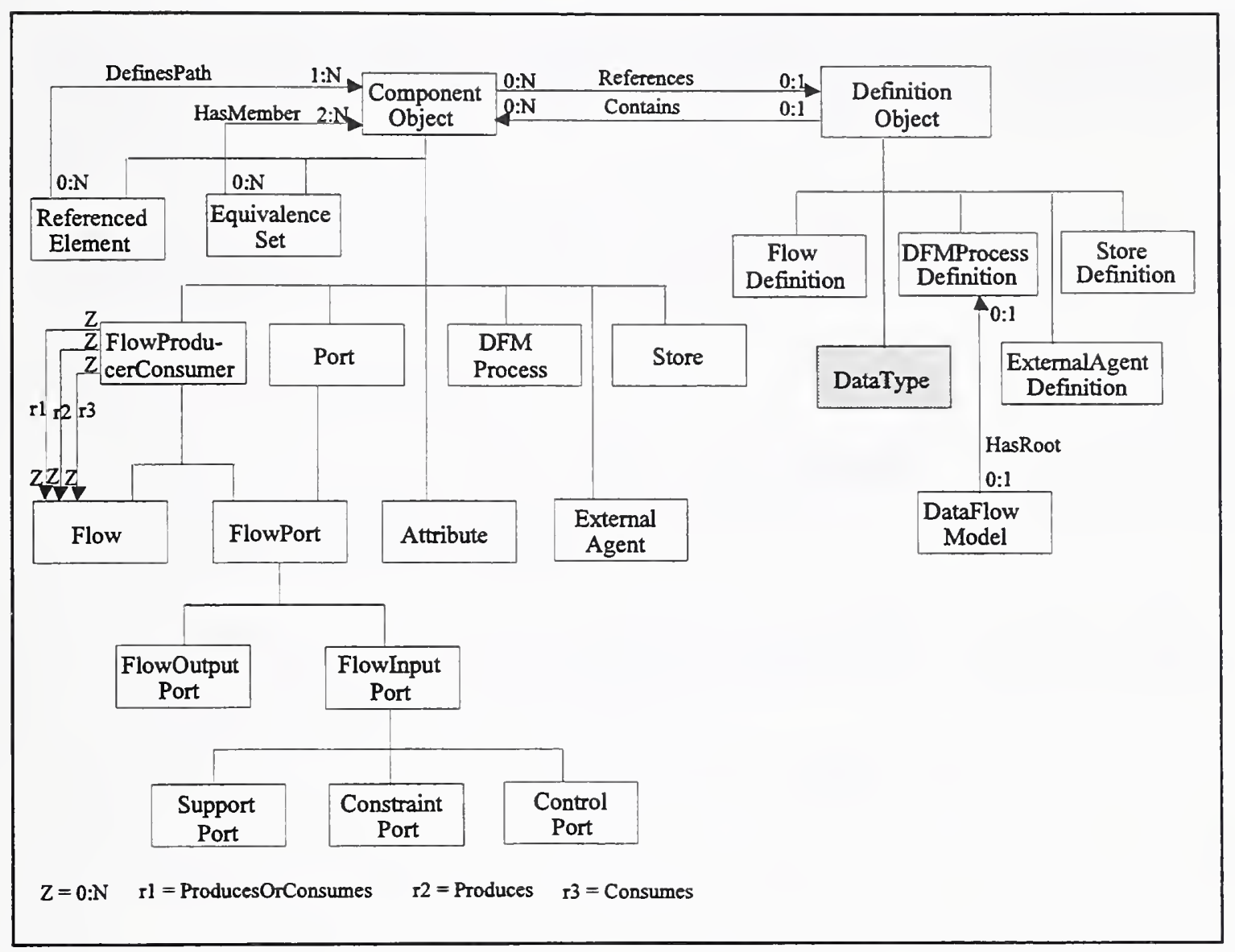

Figure 21: CDIF Data Flow Model Subject Area 


\section{APPENDIX B: Alternative Solutions}

Node References can be captured using the meta-attribute ContextIdentifier of the DFMProcess.

Process Names can be captured using the meta-attribute ContextDescription or Name of the DFMProcess. 


\section{APPENDIX C: Transfer Summary}

Any model can be transferred using CDIF Family of Standards. Even if there exists a construct in this model that is not "built in" into the CDIF Integrated Meta-Model, it can be transferred using the CDIF Framework for Modeling and Extensibility.

This research shows that $99 \%$ of IDEF0 semantics CAN be transferred using CDIF Family of Standards, and it is built in into the Integrated Meta-Model. The only major syntactical elements of IDEF0 that CANNOT map one-to-one to the CDIF Integrated Meta-Model are $\mathrm{KIT}, \mathrm{MODEL}$, and DIAGRAM.

The following CDIF Meta-meta-entities are needed to be added (or modified) to the specified subject areas to meet the requirements of the 100\% mapping of IDEF0 into a CDIF transfer file.

\section{Semantics (CDIF DFM Subject Area)}

1. A local meta-attribute "Sequence Number" (type: INTEGER) for the meta-relationship "ReferencedElement.DefinesPath.ComponentObject". It is needed to specify the correct order of objects in the path.

2. A local meta-attribute "ReferenceDescription" (type: TEXT) for the meta-entity "DFMProcess". It is needed for "A Document" IDEF0 Call -- a type of a call which references a document (book, standard, etc.)

\section{Syntax (CDIF Presentation Location and Connectivity Subject Area)}

3. A meta-entity "Kit" (with all the required meta-attributes to be mapped to the KIT IDL).

4. A meta-entity "Model" (with all the required meta-attributes to be mapped to the MODEL IDL)

5. A meta-entity "Diagram" (with all the required meta-attributes to be mapped to the DIAGRAM IDL)

6. CDIF error: the meta-attribute "ExtentX" is of type Integer, while the meta-attribute "ExtentY" is BitMap. Correction: make the meta-attribute "Extent $Y$ " also be an Int.

7. Local meta-attributes for the meta-entity "Node", which will match to BOX IDL.

8. CDIF error: no presence of the meta-attribute "Arrow Orientation" for the meta-entity "EdgeElement". Correction: list this meta-attribute in the "fat/thin" CDIF pages.

9. Local meta-attribute "Tunnel" (type: BOOLEAN) for the meta-entity "EdgeElement". 


\section{REFERENCES}

1. FIPS, Integration Definition for Function Modeling (IDEF0), FIPS PUB 183, National Institute of Standards and Technology, Gaithersburg, MD, December 1993.

2. EIA, CDIF - CASE Data Interchange Format - Overview, EIA/IS-106, January 1994.

3. EIA, CDIF - Integrated Meta-model -- Common Subject Area, EIA/PN-3070, CDIFDRAFT-CMMN-V15, Editor: Bob Matthews, May 1994.

4. EIA, CDIF - Integrated Meta-model -- Data Flow Model Subject Area, EIA/PN-3073, CDIF-DRAFT-DFM-V9, Editor: Bob Matthews, March 1995.

5. EIA, CDIF - Integrated Meta-model -- Data Definition Subject Area, EIA/PN-3071, CDIF-DRAFT-DDEF-V11, Editor: Adrian Blakey, November 1994.

6. EIA, CDIF - Framework for Modeling and Extensibility, EIA/IS-107, January 1994. 

